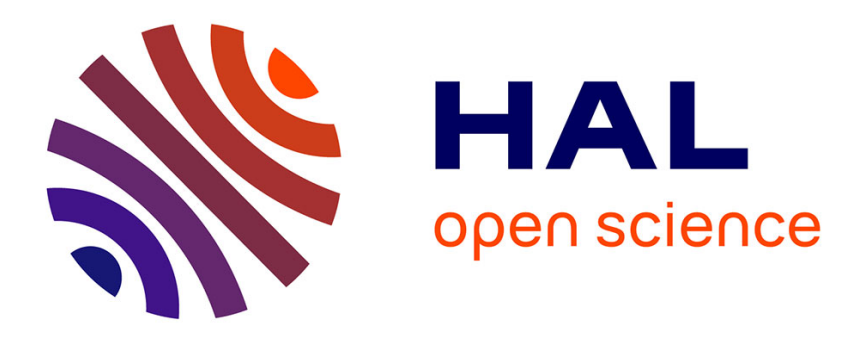

\title{
Le silicium nanoporeux : microstructuration diélectrique et application aux structures photoniques avancées
}

\author{
Patrick Ferrand
}

\section{To cite this version:}

Patrick Ferrand. Le silicium nanoporeux: microstructuration diélectrique et application aux structures photoniques avancées. E. Suraud. EDP Sciences, 27 (2), pp.121, 2002, Annales de Physique, 2-86883-608-9. 10.1051/anphys:2002002 . hal-00272896

\section{HAL Id: hal-00272896 https://hal.science/hal-00272896}

Submitted on 27 Sep 2015

HAL is a multi-disciplinary open access archive for the deposit and dissemination of scientific research documents, whether they are published or not. The documents may come from teaching and research institutions in France or abroad, or from public or private research centers.
L'archive ouverte pluridisciplinaire HAL, est destinée au dépôt et à la diffusion de documents scientifiques de niveau recherche, publiés ou non, émanant des établissements d'enseignement et de recherche français ou étrangers, des laboratoires publics ou privés. 


\section{LE SILIIIUM NANOPOREUX : MICROSTRUCTURATION}

\section{DIÉLECTRIQUE ET APPLICATION AUX STRUCTURES}

\section{PHOTONIQUES AVANCÉES}

P. Ferrand
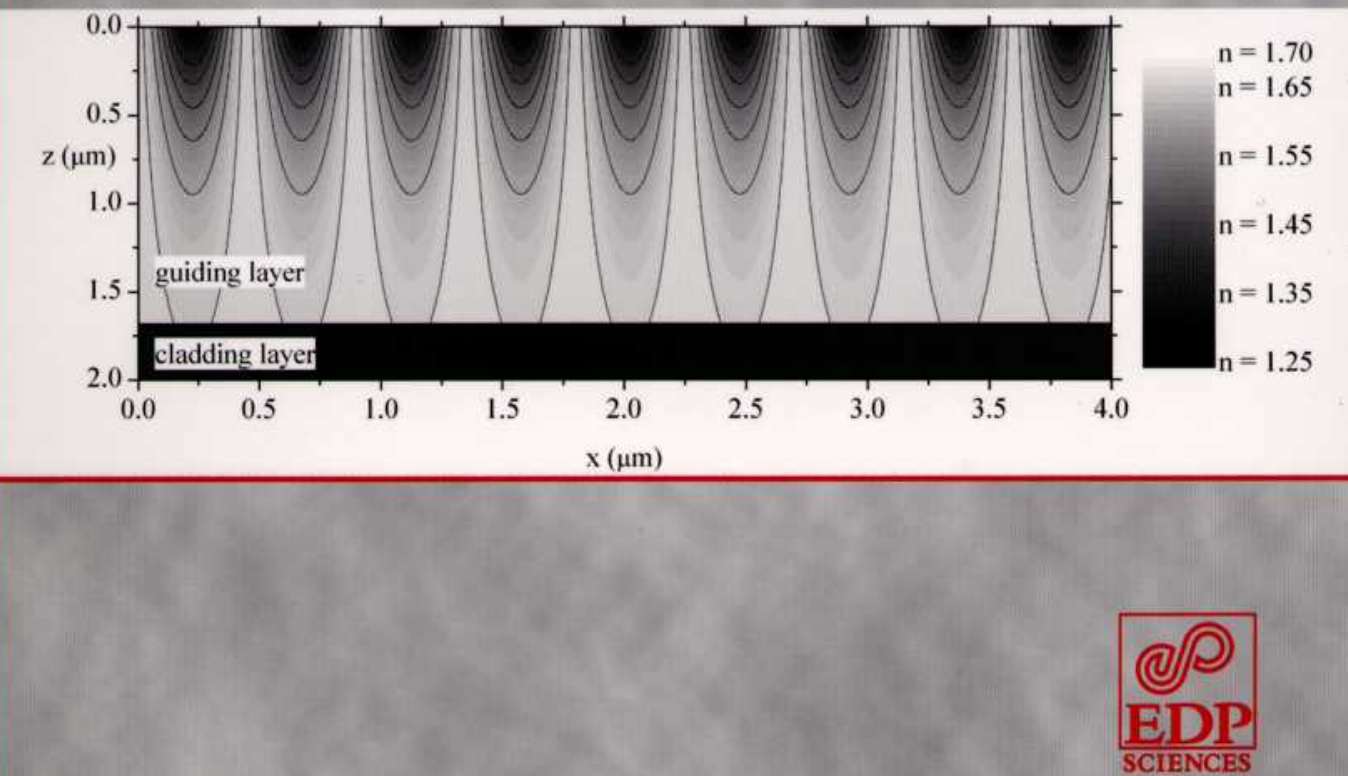


\section{Sommaire}

\section{Le silicium nanoporeux : microstructuration diélectrique et application aux structures pho- toniques avancees \\ P. Ferrand}

1 Introduction

2 Optique dans les milieux matériels $\quad 7$

1. Milieu homogène $\quad 7$

2. Généralisation à des structures multicouches 9

3. Propagation guidée 9

4. Bande interdite photonique 12

3 Présentation du silicium poreux 15

1. Formation 15

2. Propriétés 17

3. Méthodes de structuration 22

4 Méthodes expérimentales $\quad 27$

1. Élaboration des échantillons $\quad 27$ 
2. Caractérisations optiques par la surface 31

3. Caractérisations optiques en géométrie guidée 34

5 Émission de structures planaires $\quad 43$

1. Calculs d'émission spontanée 43

2. Émission dans une couche simple 48

3. Influence de la structure optique : exemple d'une microcavité

6 Structures guidantes $\quad 57$

1. Différents types de pertes 57

2. Calcul des pertes 60

3. Étude d'un guide à saut d'indice 68

4. Étude d'un guide à bande interdite photonique 76

7 Contrôle de la propagation guidée $\quad 87$

1. Principe 87

2. Méthode des modes couplés 89

3. Description de l'échantillon 92

4. Mesures de transmission 94

5. Discussion 97

8 Conclusion

Annexes 


\section{Le silicium nanoporeux : microstructuration diélectrique et application aux structures photoniques avancées}

P. Ferrand ${ }^{1}$

\section{Résumé}

Ce travail est consacré à la réalisation et à l'étude de microstructures photoniques à base de silicium nanoporeux.

Nous commençons notre étude par des structures planaires de type microcavité, dont nous caractérisons l'influence sur la photoluminescence du matériau, en termes de redistribution spectrale et angulaire. Il apparait très vite la nécessité de contrôler la propagation de la lumière dans le plan de la structure.

Aussi, nous cherchons d'abord à favoriser la propagation latérale au moyen d'une structuration verticale de l'indice, et nous étudions le guidage au moyen de deux types de structures, exploitant soit à un guidage conventionnel par réflexion totale interne, soit à un guidage par réflexion de Bragg. À cette occasion nous proposons une méthode numérique, basée sur le formalisme des matrices de transfert, permettant de calculer l'atténuation de la puissance transportée dans le plan.

Par la suite, nous mettons à profit le procédé holographique de structuration d'indice démontré par des travaux antérieurs et étudions son influence sur la lumière guidée. La transmission, mesurée en lumière blanche sur un guide multimode révèle de multiples bandes interdites que nous interprétons en termes de couplages diagonaux et non diagonaux. La confrontation des mesures avec une modélisation par la méthode des modes couplés nous permet d'établir une carte d'indice de notre structure. Il apparaît une biréfringence marquée dans les régions insolées par le procédé holographique, caractérisées par une diminution deux fois plus importante de l'indice extraordinaire $(\Delta n=-0,4)$ que de l'indice ordinaire $(\Delta n=-0,22)$. Avec une période de $450 \mathrm{~nm}$, ces valeurs de contraste sont encourageantes, bien que la modulation d'indice ne soit présente que sur une profondeur effective de l'ordre de $0,5 \mu \mathrm{m}$.

1. Laboratoire de Spectrométrie Physique, Université J. Fourier Grenoble 1, CNRS UMR 5588, B.P. 87, 38402 Saint-Martin d'Hères, France.

Adresse actuelle : Institute of Materials Science and Department of Electrical and Information Engineering, University of Wuppertal, Gauss-Str. 20, 42097 Wuppertal, Germany. 


\section{Abstract \\ Nanoporous silicon: dielectric microstructuring and application to advanced photonic structures}

The aim of this work is to elaborate and study photonic bandgap microstructures using nanoporous silicon.

Planar microstructures like microcavities are first considered, and their influence on both angular and spectral distributions of photoluminescence are investigated. The primary conclusion derived from these studies is that it is essential to control the propagation of light in the plane, too.

Thus, the lateral propagation of light, enhanced by a vertical structuring of the optical index (step-index waveguide as well as Bragg reflection waveguide) is studied. Furthermore, a numerical model based on the standard transfer-matrix method is suggested to calculate guiding losses.

Finally, a holographic process is utilized to obtain a lateral structuring of the optical index, thereby allowing the investigation of its effects on the guided light. The transmittance, measured on a multimode waveguide using white light, shows several stopbands, which are attributed to diagonal and off-diagonal couplings. The comparison of these measurements to the coupled-mode theory allows a map of the optical index to be plotted. A strong birefringence in regions that were illuminated during the holographic process was illustrated. This suggests a stronger decrease of the extraordinary index $(\Delta n=-0.4)$ than the ordinary index $(\Delta n=-0.22)$. With a period of $450 \mathrm{~nm}$, these values of index contrast are promising, even if the effective depth on which the index is modulated is only $0.5 \mu \mathrm{m}$. 


\section{Introduction}

Plus, plus vite, plus loin. Tels pourraient être résumés en quelques mots les progrès spectaculaires accomplis durant toute la seconde moitié $\mathrm{du} \mathrm{xx}^{\mathrm{e}}$ siècle dans le domaine des sciences et techniques de l'information. Et ce n'est qu'un début. À l'aube de ce nouveau siècle, l'enjeu économique (et politique) est colossal et rien ne semble pouvoir arrêter cette troisième révolution industrielle : la vitesse et la capacité des ordinateurs continuent de suivre la loi de Moore ${ }^{1}$, tandis que les autoroutes de l'information ${ }^{2}$ n'en finissent pas de voir leur débit s'accroître, conjointement à leur développement tentaculaire. Sur ce dernier aspect, l'optique occupe déjà une place de choix. Mieux encore, elle est pressentie comme une alternative à la micro-électronique (qui constitue le cœur de nos ordinateurs), que d'aucuns redoutent de voir buter sur des limites physiques dans les prochaines décennies.

Dans ce contexte, l'apparition du concept des matériaux dits à bande interdite photonique (on les appelle aussi cristaux photoniques) a suscité de la part de la communauté scientifique un enthousiasme tel que même des quotidiens nationaux s'en sont fait l'écho récemment ${ }^{3}$. Il s'agit de matériaux transparents, généralement artificiels, dont l'indice optique est structuré de façon périodique selon une ou plusieurs directions de l'espace, ce qui leur permet de s'opposer à la traversée d'ondes électromagnétiques, dans certaines conditions, lorsque la période de structuration est du même ordre de grandeur que la longueur d'onde considérée [3]. Le principe était certes déjà connu et utilisé depuis plusieurs décennies pour réaliser des miroirs interférentiels au moyen d'empilements diélectriques périodiques [4]. Mais au-delà de la généralisation à plusieurs dimensions, le coup de génie tenait plutôt à l'analogie avec la physique des semiconducteurs, laissant entrevoir des applications prometteuses : en 1987, dans son article fondateur [5], Yablonovitch y voit un moyen de vaincre certaines limitations des lasers à semiconducteurs en termes de bruit, seuil, etc., tirant parti d'une inhibition de

1. Cette loi, du nom de Gordon Moore, cofondateur de la firme Intel, prévoit un doublement de la capacité des microprocesseurs tous les 18 mois environ [1] (ou réédition [2]). Depuis 1975, elle n'a jamais été démentie. Elle s'observe également sur le coût du stockage sur disque dur qui, à capacité égale, chute de moitié pendant cette même période tandis que la densité du stockage double.

2. On doit cette métaphore à $\mathrm{Al} \mathrm{Gore,} \mathrm{lors} \mathrm{d'un} \mathrm{discours} \mathrm{prononcé} \mathrm{en} \mathrm{1993.} \mathrm{Il} \mathrm{était} \mathrm{alors} \mathrm{vice-président}$ des États-Unis. C'était en outre le thème du sommet du G7 qui s'est tenu en février 1995 à Bruxelles. 3. Cf. par exemple la pleine page consacrée aux cristaux photoniques dans Le Monde daté du 2 février 2001

Ann. Phys. Fr. 27 • No $2 \bullet 2002$ 
l'émission spontanée, prédite quarante ans auparavant par Purcell [6], mais appliquée à une échelle microscopique. Plus tard, les opportunités offertes pour la réalisation de circuits optiques de taille réduite à l'extrême [7], ou la mise en évidence de propriétés réfractives anomales [8,9], allaient jeter les bases d'un nouveau pan de la physique contemporaine. L'intérêt pour les cristaux photoniques ne réside pas seulement dans leurs applications potentielles : même si de nombreuses analogies formelles avec les propriétés électroniques des semiconducteurs sont évoquées, ils constituent bel et bien une nouvelle voie à explorer.

Les obstacles sont cependant nombreux. D'un point de vue théorique, on se heurte à des calculs très lourds dès lors que l'on prend en compte les trois dimensions de l'espace. D'un point de vue expérimental, la fabrication des cristaux photoniques est confrontée principalement à deux exigences : un fort contraste $\mathrm{d}^{\prime}$ indice $^{4}$ et une structuration de période équivalente à la longueur d'onde étudiée. C'est à cause de cette dernière contrainte que les premières structures tridimensionnelles ont été réalisées pour le domaine des micro-ondes. En ce qui concerne l'optique, la période requise (inférieure au micron) est une contrainte majeure, et les premières réalisations ont dû attendre les récents progrès accomplis dans les techniques lithographiques, et se contenter d'une structuration limitée aux deux dimensions du plan [10]. À l'heure actuelle, mis à part des assemblages de type tas de bois [11], très lourds à mettre en œuvre, seules les opales ${ }^{5}$ constituent des structures véritablement tridimensionnelles $[12,13]$, mais la régularité à grande échelle reste encore un problème.

Pour ces raisons, de plus en plus de groupes s'orientent désormais vers des matériaux alternatifs, pour lesquels les contrastes d'indices accessibles sont certes parfois moins élevés que dans le cas des semiconducteurs, mais suffisants pour des structures confinées verticalement par guidage conventionnel. Ils offrent souvent une plus grande souplesse de fabrication.

Le silicium nanoporeux appartient de toute évidence à cette catégorie : les indices accessibles en jouant sur la porosité sont certes notablement moins élevés que dans le cas du silicium massif, mais les procédés de fabrication sont simples et offrent la possibilité de différents types de structurations. La luminescence à température ambiante, dans certaines conditions, et la capacité à héberger des émetteurs, constituent d'autres propriétés enviables et ont été largement exploitées par le passé, en conjugaison des effets de filtrage et d'extraction apportés par des structures verticales de type microcavité [14].

Par contre, la structuration périodique de l'indice dans le plan, rendue possible par un procédé de gravure holographique relativement simple [15], n'a jamais fait l'objet d'études optiques, excepté en termes de diffraction par la surface, ignorant ainsi une opportunité de contrôler l'émission de lumière dans le plan, fléau des structures à émission de surface.

4. Le fort contraste d'indice est une condition nécessaire pour que la bande interdite soit commune à un maximum de directions.

5. Les opales sont, à l'état naturel, des pierres dont les reflets irisés sont dus aux interférences produites par l'empilement compact des microbilles siliceuses qui les composent. Elles constituent le seul exemple de cristal photonique tridimensionnel naturel. On sait réaliser ces empilements de façon artificielle et contrôlée. 
C'est à cette propagation dans le plan qu'est consacré cet ouvrage, et plus particulièrement aux possibilités de la contrôler par une structuration périodique. Avec cette perspective, nous avons choisi dans un premier temps de structurer verticalement le matériau, afin de donner lieu à un transport de lumière efficace dans le plan, par effet de guidage, puis nous avons réalisé une structuration périodique limitée, pour simplifier le problème, à une seule direction du plan, direction selon laquelle nous avons étudié la propagation de lumière guidée.

Dans le chapitre 2, nous formulerons quelques rappels d'optique des milieux matériels structurés. Nous insisterons particulièrement sur la notion de mode guidé, qui interviendra de façon omniprésente dans l'ensemble de l'ouvrage, ainsi que sur l'apparition de bande interdite photonique résultant d'une structuration périodique.

Le troisième chapitre sera consacré au silicium poreux en général. L'accent sera mis particulièrement sur ses propriétés optiques et son aptitude à la structuration.

Le chapitre 4 décrira les méthodes expérimentales utilisées à la fois pour l'élaboration et pour les caractérisations optiques. Un accent particulier sera mis sur la configuration expérimentale utilisée en optique guidée pour des caractérisations, soit en lumière blanche, soit en exploitant l'émission intrinsèque du matériau.

Le chapitre 5 sera le point de départ de notre étude : à titre préliminaire, nous nous intéresserons à l'émission de lumière, dans le cadre général des structures planaires, l'idée sous-jacente étant la caractérisation de l'émission intrinsèque du silicium, le procédé de fabrication par gravure pouvant faire craindre (ou espérer) une disproportion entre le nombre de dipôles horizontaux et verticaux, ces derniers n'intervenant que dans l'émission dans le plan. Une méthode classique de calcul de l'émission spontanée sera d'abord présentée, puis nous détaillerons les mesures d'émission verticale réalisées sur des échantillons de silicium nanoporeux bénéficiant d'une structuration uniquement verticale. Les propriétés de l'émission intrinsèque, les effets en termes de redistribution spectrale et angulaire, efficacité d'extraction, seront également analysés et discutés.

Le sixième chapitre sera consacré à la réalisation et à l'étude de structures permettant un guidage dans le plan. Nous évoquerons les différentes sources de pertes susceptibles de limiter le guidage et proposerons une méthode numérique générale permettant de les évaluer quantitativement. Deux types de structures guidantes, exploitant soit un guidage conventionnel à saut d'indice soit un guidage par bande interdite photonique, seront décrits et analysés en termes de gamme spectrale, de pertes, et d'émission guidée.

Enfin, dans le chapitre 7, nous utiliserons cette propagation guidée pour sonder une structuration périodique de l'indice dans le plan. Nous commencerons par exposer les points clés de la méthode des modes couplés, puis nous analyserons les mesures de transmission réalisées en lumière blanche et nous nous intéresserons aux conséquences en termes d'émission de surface.

L'ensemble des résultats obtenus nous permettra d'évaluer les potentialités du silicium nanoporeux en tant que matériau à bande interdite photonique, tant d'un point de vue fondamental qu'appliqué. 


\section{Optique dans les milieux matériels}

Nous rappelons dans ce chapitre les principes de base qui gouvernent la propagation des ondes électromagnétiques dans les milieux diélectriques. Nous traitons également deux situations particulières : la propagation guidée et la propagation dans un milieu périodique.

\section{Milieu homogène}

\subsection{Ondes planes}

Comme tout phénomène électromagnétique en général, l'optique est gouverné par les quatre équations de Maxwell [16]. Dans un milieu homogène, isotrope, non-magnétique, de constante diélectrique complexe $\epsilon$ et en l'absence de sources, elles s'écrivent en unités SI

$$
\begin{array}{ll}
\nabla \wedge \mathbf{E}=-\mu_{0} \frac{\partial \mathbf{H}}{\partial t}, & \nabla \cdot \mathbf{E}=0 \\
\nabla \wedge \mathbf{H}=\epsilon_{0} \epsilon \frac{\partial \mathbf{E}}{\partial t}, & \nabla \cdot \mathbf{H}=0
\end{array}
$$

où l'on désigne par $\mathbf{E}$ et $\mathbf{H}$ les champs électrique et magnétique, et où $\epsilon_{0}$ et $\mu_{0}$ représentent la permittivité et la perméabilité magnétique du vide. En combinant ces équations, on montre (i) que chacun des champs vérifie une équation d'onde, (ii) qu'ils sont reliés par une relation très simple. Aussi, nous nous bornerons à exprimer les relations à venir en fonction du champ électrique, dont l'équation d'onde

$$
\nabla^{2} \mathbf{E}-\mu_{0} \epsilon_{0} \epsilon \frac{\partial^{2} \mathbf{E}}{\partial t^{2}}=0
$$

admet comme solutions des ondes planes de la forme ${ }^{1}$

$$
\mathbf{E}(\mathbf{r}, t)=\mathbf{E} \exp [\mathrm{i}(\mathbf{k} \cdot \mathbf{r}-\omega t)],
$$

1. Dans cette expression ainsi que dans celles qui suivent, le champ E est une grandeur complexe, dont la partie réelle constitue le champ physique. 
de longueur d'onde dans le vide $\lambda=2 \pi c / \omega$, de fréquence $v=\omega / 2 \pi$, et dont le vecteur d'onde $\mathbf{k}$ est défini de telle sorte que $(\mathbf{E}, \mathbf{H}, \mathbf{k})$ est un trièdre direct et

$$
k^{2}=n^{2} \frac{\omega^{2}}{c^{2}}
$$

où $n \equiv \sqrt{\epsilon}$ et $\mu_{0} \epsilon_{0} c^{2} \equiv 1$. En outre, la moyenne temporelle du flux d'énergie de cette onde à travers une surface fermée est donné par le flux à travers cette même surface de la partie réelle $\mathbf{P}$ du vecteur de Poynting complexe, soit

$$
\mathbf{P}=\frac{1}{2} \operatorname{Re}\left(\mathbf{E} \wedge \mathbf{H}^{*}\right)
$$

Les ondes planes revêtent une importance particulière dans la mesure où elles constituent une base commode sur laquelle peut être développée toute onde électromagnétique. En outre, les équations qui régissent leur comportement à l'interface de deux milieux sont simples et peuvent être résolues rigoureusement.

\subsection{Interface de deux milieux homogènes}

Considérons l'interface entre deux milieux d'indices $n_{1}$ et $n_{2}$, telle qu'elle est représentée sur la figure 1 . Dans la mesure où notre système est invariant selon les deux directions $\hat{\mathbf{x}}$ et $\hat{\mathbf{y}}$, les conditions aux limites doivent être satisfaites à tout instant et sur toute l'interface. Ceci impose les mêmes variations spatiale et temporelle du champ électrique en tout point du plan $x y$, donc la conservation des composantes tangentielles des vecteurs d'onde. Aussi, nous pouvons écrire l'amplitude du champ électrique sous la forme $E(\mathbf{r}, t)=E(z) \exp \left[i\left(k_{x} x+k_{y} y-\omega t\right)\right]$, et dans chaque milieu $i$, on montre facilement que $E(z)$ peut s'écrire comme la somme de deux ondes se propageant dans le sens $+z(\uparrow)$ ou $-z(\downarrow)$, selon

$$
E_{i}(z)=E_{i \uparrow} \exp \left(+\mathrm{i} k_{z, i} z\right)+E_{i \downarrow} \exp \left(-\mathrm{i} k_{z, i} z\right)=\left(\begin{array}{c}
E_{i \uparrow} \\
E_{i \downarrow}
\end{array}\right) \text {. }
$$

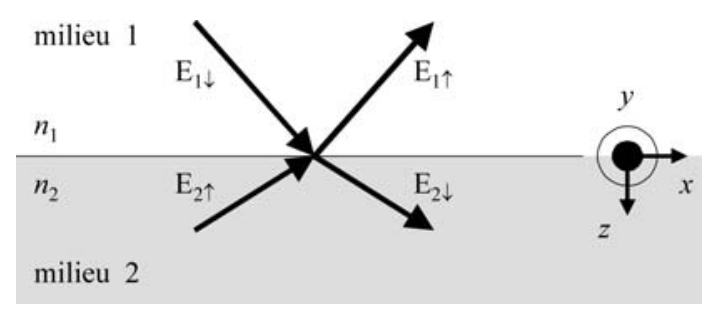

Figure 1. Ondes planes (chaque flèche représente le vecteur d'onde) de part et d'autre d'une interface. Les amplitudes des champs électriques associés sont indiquées près de chaque flèche. Dans le cas présent, $n_{1}>n_{2}$.

[Plane waves (arrows represent wave vectors) at the interface between two semi-infinite media. Corresponding amplitudes of fields are indicated close to each arrow. In the present case, $n_{1}>n_{2}$.] 
Les conditions de continuité des champs électromagnétiques permettent de relier les valeurs des amplitudes de part et d'autre de l'interface. En polarisation TE (lorsque E est le long de $\hat{\mathbf{y}}$ ),

$$
\left\{\begin{array}{rl}
\left(E_{1 \uparrow}+E_{1 \downarrow}\right) & =\left(E_{2 \uparrow}+E_{2 \downarrow}\right) \\
k_{z, 1}\left(E_{1 \uparrow}-E_{1 \downarrow}\right) & =\left(E_{2 \uparrow}-E_{2 \downarrow}\right) k_{z, 2}
\end{array},\right.
$$

et en polarisation TM (lorsque E est dans le plan $x z$ ),

$$
\left\{\begin{array}{rl}
k_{z, 1} / k_{1}\left(E_{1 \uparrow}+E_{1 \downarrow}\right) & =\left(E_{2 \uparrow}+E_{2 \downarrow}\right) k_{z, 2} / k_{2} \\
k_{1}\left(E_{1 \uparrow}-E_{1 \downarrow}\right) & =\left(E_{2 \uparrow}-E_{2 \downarrow}\right) k_{2}
\end{array} .\right.
$$

Étant donné l'équation (2.4), il apparaît que les couples $\left(E_{1 \uparrow}, E_{1 \downarrow}\right)$ et $\left(E_{2 \uparrow}, E_{2 \downarrow}\right)$ sont liés de façon biunivoque par l'équation (2.7) (ou (2.8), suivant le polarisation).

\section{Généralisation à des structures multicouches}

Les équations $(2.7,2.8)$, écrites sous forme matricielle, constituent les briques majeures du formalisme dit des matrices de transfert. Cette méthode analytique est utilisée pour calculer le champ électrique en tout point d'une structure multicouche, au moyen d'une succession d'opérations matricielles élémentaires².

Outre sa rigueur, la puissance de cette méthode réside dans sa généralité : aucune restriction n'est faite sur les valeurs possibles du vecteur d'onde ou des indices, si bien qu'elle peut décrire une grande variété de situations. Très efficace pour calculer la réflectivité, la transmission de structures multicouches (réflecteurs de Bragg, microcavités, etc.), nous verrons qu'elle peut s'adapter à des situations moins conventionnelles, pour calculer par exemple la modification de l'émission d'un dipôle dans une structure arbitraire ( $c f . \S 1.2$. ., Chap. 5) ou les fuites d'un mode de guide (cf. § 2., Chap. 6).

\section{Propagation guidée}

\subsection{Réflexion totale}

Les relations de continuité ont pour conséquence que les champs ne dépendent de l'indice du milieu que par le biais de la composante du vecteur d'onde qui est normale à l'interface. Dans la configuration de la figure 1, en posant $k_{0} \equiv \omega / c$, d'après l'équation (2.4), elle vérifie

$$
\beta^{2}+k_{z, i}^{2}=k_{0}^{2} n_{i}^{2},
$$

où nous avons désigné par $\beta$ la composante tangentielle du vecteur d'onde (aussi appelée constante de propagation) pour nous conformer aux usages de l'optique

2. L'ouvrage de Yeh [4] est une référence en la matière. 
guidée. Il apparaît en particulier que si $\beta>k_{0} n_{i}$, alors $k_{z, i}$ est imaginaire pur, ce qui interdit la propagation de l'onde suivant $\hat{\mathbf{z}}$ dans le milieu d'indice $n_{i}$.

Lorsqu'une onde plane attaque l'interface d'un milieu semi-infini dans lequel sa constante de propagation ne l'autorise pas à se propager, elle subit une réflexion totale. Dans le milieu «interdit », le champ électromagnétique décroît exponentiellement sur une profondeur de même ordre de grandeur que la longueur $\mathrm{d}$ 'onde ; l'onde est dite évanescente et le transport d'énergie se fait uniquement au voisinage de l'interface, parallèlement à celle-ci.

\subsection{Notion de mode guidé}

Le guidage est un moyen efficace de transporter de l'énergie, en confinant la lumière dans un milieu, grâce à des réflexions totales contre des matériaux voisins d'indice plus faible. Néanmoins, la condition de réflexion totale n'est pas suffisante pour permettre le guidage : on montre facilement que les conditions de continuité des champs aux interfaces ne peuvent être vérifiées que pour un ensemble discret et fini de valeurs de $\beta$ qui constituent les modes du guide. Dans la mesure où chaque mode guidé se comporte comme une onde plane dans la direction longitudinale du guide, on a coutume de donner pour chaque mode son indice effectif $n_{\text {eff, }}$ défini $\operatorname{par} \beta=k_{0} n_{\text {eff. }}$.

En outre, chaque mode possède sa propre distribution de champ électrique, comme l'illustre la figure 2 , sur laquelle est schématisé un guide planaire asymétrique, d'épaisseur $e_{2}$, avec $n_{1}<n_{3}<n_{2}$.

\subsection{Dispersion}

Lorsque l'étude d'une structure guidante couvre une large gamme spectrale, il est utile de considérer la dispersion des modes. La courbe de dispersion, calculée pour le guide de la figure 2 est représentée sur la figure 3. Malgré les valeurs spécifiques des indices, cette figure garde un caractère de généralité. Il apparaît notamment que les modes appartiennent à une région limitée par les droites $\omega=c \beta / n_{2}$ et

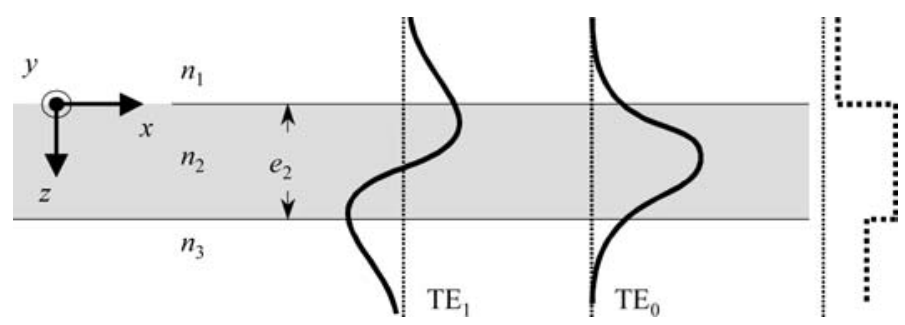

Figure 2. Vue schématique d'un guide d'onde planaire. La distribution du champ électrique des deux premiers modes $\mathrm{TE}_{0}$ et $\mathrm{TE}_{1}$ est reportée, ainsi que le profil d'indice (en pointillés). [Scheme of a planar waveguide. The distribution of electrical field for the two first so-called $\mathrm{TE}_{0}$ et $\mathrm{TE}_{1}$ modes is plotted, as well as the index profile (dotted line).] 


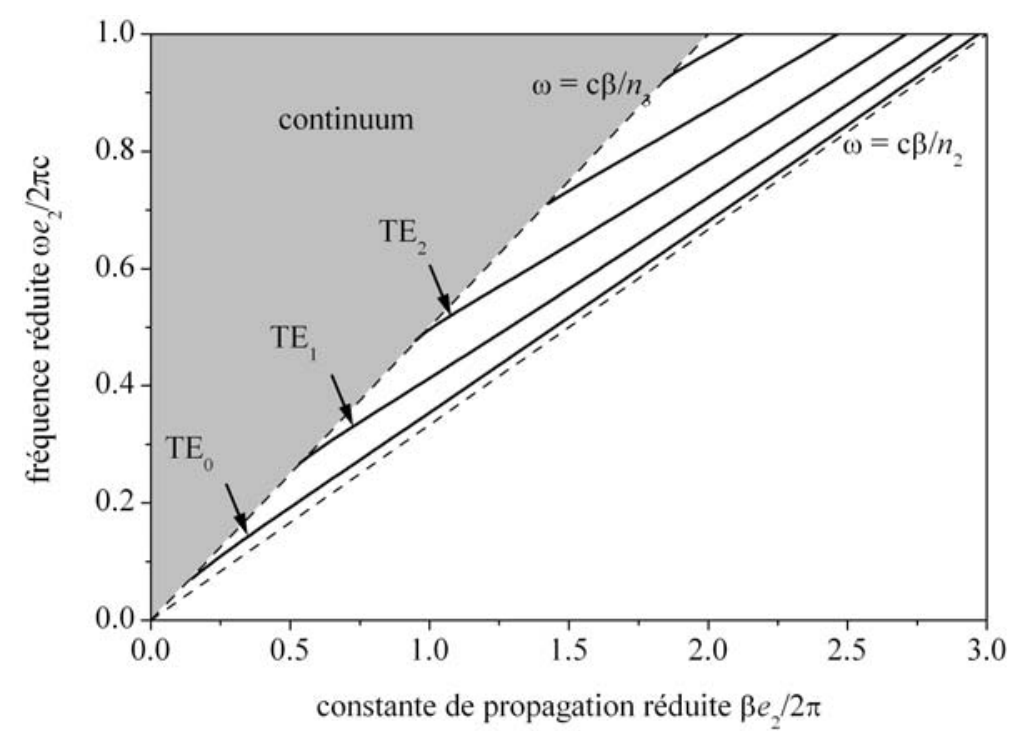

Figure 3. Dispersion d'un guide d'onde planaire asymétrique tel que celui qui est dessiné sur la figure 2. Les indices choisis ici sont $n_{1}=1, n_{2}=3, n_{3}=2$, et seule la polarisation TE est représentée. Les premiers modes guidés sont indiqués par des flèches. Les droites en pointillés indiquent les limites du domaine d'existence des modes guidés.

[Dispersion of a typical planar asymmetric waveguide, as described in Figure 2. Index values are $n_{1}=1, n_{2}=3, n_{3}=2$, and only TE polarization is plotted. The first guided modes are indicated by arrows. Guided modes can only exist between the two dotted lines.]

$\omega=c \beta / n_{3}$, qui correspondent respectivement à une propagation rasante dans la couche guidante, et à la limite de réflexion totale contre le matériau environnant d'indice $n_{3}$. La figure met clairement en évidence l'augmentation progressive du nombre de modes pouvant être guidés dans la structure, lorsque la fréquence augmente. Quant à la zone grisée, elle correspond à un continuum de modes dits rayonnants qui, en l'absence de confinement par réflexion totale sur les deux interfaces, se propagent au-delà de la couche guidante.

\subsection{Relations d'orthogonalité}

Il est possible de montrer que dans le cas d'un guide non absorbant, les modes guidés sont orthogonaux aux modes du continuum et orthogonaux entre eux [17]. Pour notre guide planaire, cette dernière propriété s'écrit

$$
\int_{-\infty}^{+\infty}\left[\mathbf{E}_{i} \wedge \mathbf{H}_{j}^{*}\right] \cdot \hat{\mathbf{x}} \mathrm{d} z=\int_{-\infty}^{+\infty}\left[\mathbf{E}_{j}^{*} \wedge \mathbf{H}_{i}\right] \cdot \hat{\mathbf{x}} \mathrm{d} z=0, \quad i \neq j,
$$

où $i$ et $j$ indexent les modes guidés. 


\subsection{Couplage par la tranche}

Le moyen le plus conventionnel pour injecter de la lumière dans un guide consiste à focaliser un faisceau sur la tranche du guide. Il s'agit d'un processus éminemment complexe (les effets de bords jouent ici un rôle majeur) ; on retiendra cependant que

- l'ouverture angulaire du faisceau incident doit être inférieure à l'ouverture effective du guide ;

- la distribution de champ de l'onde incidente est décomposée sur la base des modes (guidés et radiatifs) du guide; dit autrement, le couplage avec un mode sera d'autant plus efficace que la figure projetée sur la tranche du guide correspondra à son profil de puissance transportée.

\section{Bande interdite photonique}

Dans le cas d'un milieu dont la constante diélectrique $\epsilon(\mathbf{r})$ varie de façon périodique (on emploie alors le terme de cristal photonique), les équations de Maxwell se réduisent à une équation aux valeurs propres [3] en $\mathbf{H}$, similaire à l'équation de Schrödinger que vérifie la fonction d'onde d'un électron dans le potentiel périodique d'un réseau cristallin d'ions.

Cette analogie permet l'utilisation des outils cristallographiques développés pour la physique du solide : on montre notamment que les solutions sont des ondes de Bloch [18]. Mais l'intérêt des milieux périodiques réside sans conteste dans l'apparition possible de plages d'énergie appelées bandes interdites photoniques pour lesquelles la propagation des photons est interdite.

\subsection{Milieu périodique unidimensionnel}

Le milieu périodique unidimensionnel est un cas d'école. Il a été étudié très tôt : Kossel [19] a étudié ses similitudes avec la physique du solide en 1966, et Yeh et al. [20] l'ont formulé en terme de matrices de transfert quelques années plus tard. Il a le mérite d'illustrer la notion de bande interdite photonique de façon particulièrement intuitive, $d$ 'autant que les calculs peuvent être menés au moyen de méthodes numériques relativement rudimentaires.

Nous raisonnerons dans un premier temps sur une structure finie, que l'on appelle miroir de Bragg : il s'agit d'un empilement périodique de couches diélectriques d'indices $n_{1}$ et $n_{2}$ de période $\Lambda$, comme le montre la figure 4 a. Pour une onde plane en incidence normale (selon l'axe $\hat{\mathbf{z}}$ sur la figure), on montre que les réflexions multiples sur les interfaces successives sont en phase si les couches ont une épaisseur optique d'un quart de longueur d'onde. Cela se traduit dans la structure par l'établissement d'une onde stationnaire dont l'enveloppe décroît en profondeur. Expérimentalement, cette situation donne lieu à la réflexion quasitotale de l'onde incidente, dans une gamme finie de fréquences qui constitue la bande interdite, comme l'illustre la figure $4 \mathrm{~b}$. On montre en outre que la largeur de la bande interdite est proportionnelle au contraste d'indice $n_{1}-n_{2}$. 


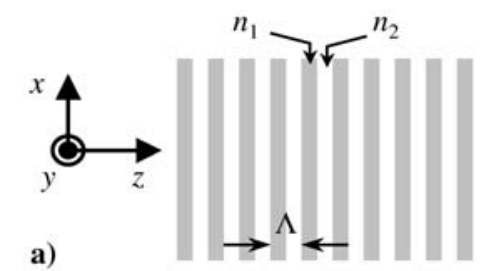

b)

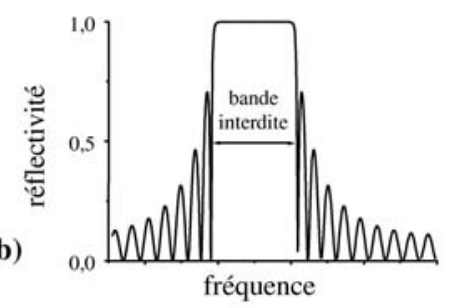

Figure 4. (a) Schéma d'un milieu diélectrique périodique fini. (b) Spectre de réflectivité typique d'un miroir de Bragg faisant apparaître une bande interdite.

[(a) Scheme of a finite periodic dielectric medium. (b) Typical reflectance spectrum of a Bragg mirror, emphasizing the stopband.]

Dans le cas d'une structure infinie, nous serions dans la gamme énergétique où les solutions de l'équation aux valeurs propres sont évanescentes, ce qui interdit la propagation d'énergie.

Sous une incidence oblique, l'épaisseur optique est modifiée, et la bande interdite est décalée à des fréquences plus élevées. En outre, sa largeur change généralement, car les relations de continuité aux interfaces dépendent de l'incidence et de la polarisation. Autrement dit, à fréquence donnée, la bande interdite possède une certaine acceptance angulaire, d'autant plus importante que le contraste d'indice est élevé.

Ce type de structuration périodique unidimensionnelle est aussi connu en optique guidée sous le nom de réseau de Bragg. Le réseau est alors gravés transversalement à la direction de guidage. De tels dispositifs sont utilisés comme filtres, et interviennent notamment dans les systèmes commerciaux de multiplexage en longueur d'onde (ou DWDM pour Dense Wavelength Division Multiplexing) [21].

\subsection{Généralisation à plusieurs dimensions}

Le cas d'un milieu périodique le long de plusieurs directions est plus complexe, même si les principes qui gouvernent l'ouverture de bande interdite sont les mêmes.

Tout l'intérêt réside dans la possibilité d'obtenir une bande interdite pour toutes les directions de propagation. À deux dimensions, on retiendra que la structure la plus simple possédant une bande interdite complète, c'est-à-dire pour toutes les directions et pour les deux polarisations est le réseau triangulaire de cylindres [23], comme l'illustre la figure 5. Elle nécessite cependant un rapport d'indice supérieur à 3. Des structures plus complexes, exploitant une symétrie d'ordre plus élevé, peuvent se contenter d'un rapport d'indice beaucoup plus faible, rendant possible l'utilisation de silice [24]. À trois dimensions, Ho et al. [25] ont été les premiers à montrer théoriquement que la structure diamant était un bon candidat. On signalera également la Yablonovite, nom donné à la structure suggérée peu de temps après par Yablonovitch et al. [26], et qui permet d'obtenir 


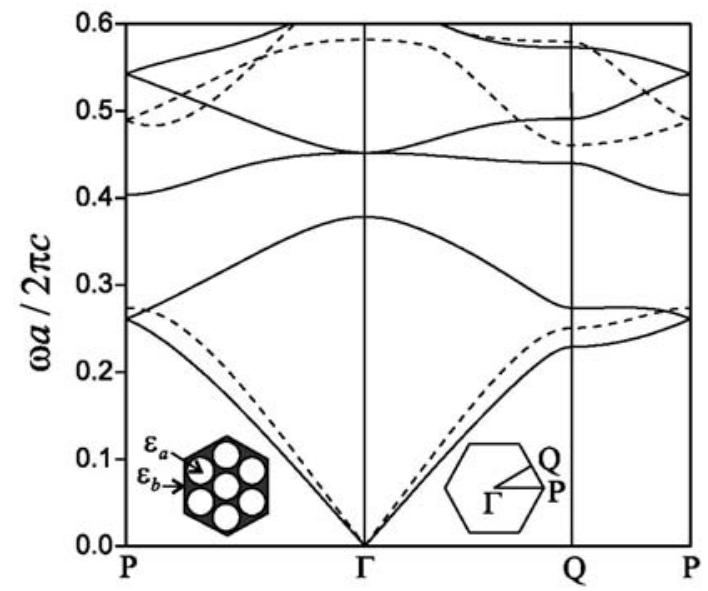

Figure 5. Exemple de structure de bandes photoniques bidimensionnelle. Le milieu est constitué d'un réseau triangulaire de cylindres infinis. La bande interdite absolue (à la fois pour les polarisations $\mathrm{TE}$, en train plein et TM, en trait pointillé) apparaît clairement pour $\omega a / 2 \pi c \simeq 0,4$. $\mathrm{D}^{\prime}$ après [22], avec la permission de D. Cassagne.

[Example of two-dimensional photonic band structure. The medium is a triangular lattice of infinite cylinders. The absolute photonic bandgap (in the mean time for the TE (solid line) and TM (dotted line)) appears clearly at $\omega a / 2 \pi c \simeq 0.4$. From [22], with the permission of D. Cassagne.]

une structure cubique à faces centrées par une astucieuse séries de perçages bien orientés.

Dans ce chapitre, nous avons vu brièvement les principes généraux qui gouvernent la propagation des ondes électromagnétiques dans les milieux matériels. Nous avons insisté sur deux configurations très différentes, qui ont cependant comme point commun d'être capables, au moins dans une région limitée de l'espace, de s'opposer sous certaines conditions à la propagation de lumière : par réflexion totale pour le guide d'onde ou par effet de bande interdite photonique pour le cristal photonique. Chaque fois, la dispersion des photons s'en trouve fortement affectée. 


\section{Présentation du silicium poreux}

Nous décrivons brièvement dans ce chapitre la formation du matériau, ainsi que ses principales propriétés ${ }^{1}$, notamment optiques. Nous insistons ensuite tout particulièrement sur les différents moyens offerts quant à sa structuration.

L'histoire du silicium poreux commence il y a presque un demi-siècle lorsque Uhlir [32] puis Turner [33] observent un film coloré de nature inconnue à la surface de leurs échantillons, en mettant au point une méthode électrochimique pour le polissage du silicium. Quelques années plus tard, les travaux de Memming et Schwandt [34] puis de Theunissen [35], montrent que sa formation résulte d'une dissolution localisée du silicium et non d'un dépôt en surface.

Dans les années qui suivent, l'étude du matériau est notamment motivée par ses potentialités concernant la réalisation de structures de type silicium sur isolant [36], mais c'est en 1990 que la découverte de la luminescence du silicium poreux à température ambiante par Canham [37] a l'effet d'une bombe : les potentialités en terme d'applications optoélectroniques, interdites au silicium en raison de son gap indirect, génèrent une croissance exponentielle du nombre de publications sur le sujet.

\section{Formation}

\subsection{Conditions de formation}

Le silicium poreux est obtenu par attaque électrochimique d'un substrat monocristallin de silicium dans une solution à base d'acide fluorhydrique (HF). Le silicium constitue l'anode, tandis qu'un métal inerte (platine) immergé dans l'électrolyte joue le rôle de la cathode. Les principaux paramètres entrant en jeu sont :

- le dopage du substrat de silicium,

- le dosage de la solution en HF,

- la densité de courant d'anodisation,

- l'illumination éventuelle.

La formation d'une couche poreuse n'est possible que dans un régime électrochimique particulier, fondamentalement différent du régime d'électropolissage.

1. Une description plus complète des processus de formation du matériau peut être trouvée dans le recueil des cours donnés à l'école des Houches [27]. Quant aux propriétés du matériau, elles font l'objet de nombreux articles de revue [28-30] et d'un ouvrage spécialisé [31]. 


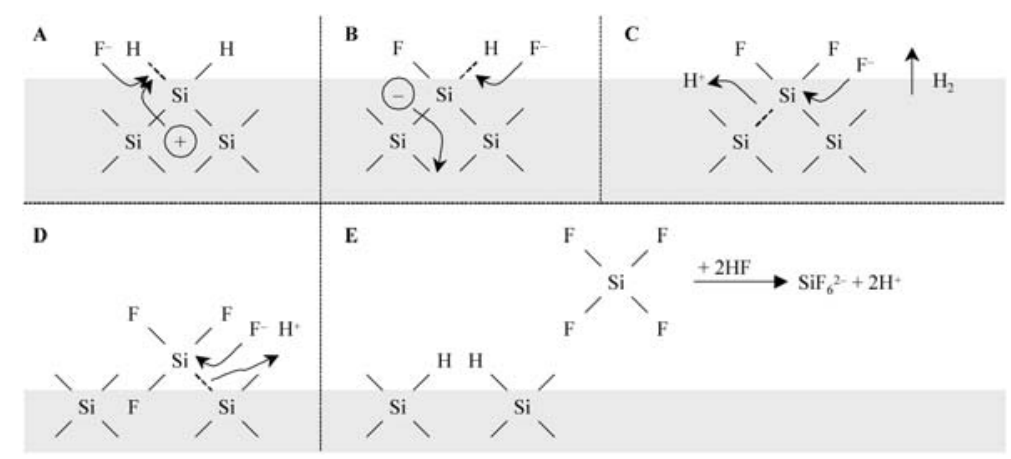

Figure 6. Étapes successives du modèle de dissolution du silicium poreux proposé par Lehmann et Gösele [38]. La région grisée représente le silicium massif. La capture d'un trou dans la première réaction justifie le rôle critique joué par le dopage du silicium.

[Successive steps of the dissolution model proposed by Lehmann and Gösele [38]. Bulk silicon is represented in grey.]

\subsection{Réactions mises en jeu}

La dissolution électrochimique du silicium est un processus d'oxydation. La séquence des différentes réactions chimiques et électrochimiques, proposée par Lehmann et Gösele [38] est reportée sur la figure 6. Il apparaît notamment que la première étape (A), qui correspond à la cassure d'une liaison $\mathrm{Si}-\mathrm{H}$ à la surface du silicium, nécessite la capture d'un trou $h^{+}$, ce qui justifie le rôle critique joué par le dopage du substrat. Les réactions électrochimiques (A et B), puis simplement chimiques (C-E) qui s'enchaînent permettent de briser progressivement les liaisons autour de chaque atome de surface, afin de le mettre en solution, sous forme d'une molécule $\mathrm{SiF}_{4}$. Le processus se répète sur les atomes suivants. La surface $\mathrm{du}$ cristal est donc alternativement recouverte de fluor (étape $\mathrm{C}$ ) et d'hydrogène (étape E). D'autres modèles ont été proposés; ils diffèrent quelque peu dans le détail des réactions, mais l'influence des paramètres extérieurs (trous, électrons), reste globalement la même [39].

\subsection{Formation des pores}

Si la dissolution, c'est-à-dire l'élimination de matière, est relativement bien comprise, les mécanismes qui sont responsables de la formations de pores sont d'une origine beaucoup plus controversée. Pourquoi l'attaque s'initie-t-elle à un endroit plutôt qu'un autre? Comment s'effectue la croissance des pores?

Aucune de ces questions n'a obtenu de réponse irréfutable. Les modèles proposés sont nombreux et variés [40], ils s'appuient tantôt sur des réactions chimiques, en raison de différences d'activité chimique en différents points de la surface du substrat, tantôt sur des phénomènes physiques, à cause de défauts électriques (effet de pointe) [41] dus à des irrégularité de la surface de silicium, ou bien à cause 
d'une instabilité du front de dissolution [42]. Tous convergent cependant vers la croissance d'un front de dissolution localisé au fond de la couche poreuse.

\subsection{Conséquences sur l'homogénéité des couches}

Nous venons de voir que le processus était limité au fond de la couche poreuse, seul endroit où coexistent le silicium, les ions fluor et les trous. Dans le cas d'une électrolyse pratiquée à densité de courant constante, on peut supposer en première approximation que les conditions électrochimiques ne varient pas au cours du temps ${ }^{2}$, et donc que la formation en régime constant donne lieu à une couche parfaitement homogène en profondeur. C'est effectivement ce qui est constaté expérimentalement : les couches font preuve d'une homogénéité très satisfaisante, tant dans le plan qu'en profondeur, et leur épaisseur est généralement proportionnelle à la durée pendant laquelle le courant a circulé.

\section{Propriétés}

\subsection{Propriétés structurales}

\section{Porosité}

La porosité est définie comme le pourcentage volumique d'air dans le matériau. Cette caractéristique macroscopique peut être mesurée par gravimétrie, de préférence sur des couches épaisses. Les valeurs accessibles en silicium poreux sont typiquement comprises typiquement entre 15 et $90 \%$.

Il est maintenant bien établi que la porosité dépend principalement de la densité de courant et du dosage de l'électrolyte. On retiendra qu'elle augmente lorsque la densité de courant augmente ou lorsque la concentration en HF diminue.

Nous ne ferons mention de la porosité de nos échantillons qu'à titre indicatif, tant les propriétés structurales et optiques peuvent différer, même à porosité identique.

\section{Morphologie}

La taille et la forme des pores sont essentiellement liées au dopage du substrat de départ. Quatre catégories de silicium poreux, illustrées sur la figure 7, sont donc traditionnellement distinguées, selon ce dopage ( $\rho$ désigne la résistivité, $N_{\mathrm{a} / \mathrm{d}}$ la concentration d'accepteurs/donneurs). Il s'agit :

- du type $p\left(\rho \sim 1 \Omega \mathrm{cm} ; N_{\mathrm{a}} \sim 10^{16} \mathrm{~cm}^{-3}\right)$ : la structure, de type éponge, est relativement isotrope (certes peu visible sur la Fig. 7a). Les cristallites, assimilables à des ellipsoïdes de taille nanométrique, sont fortement interconnectées ;

2. C'est l'appauvrissement de l'électrolyte, ou tout simplement la progression difficile des espèces chimiques dans le dédale de pores, qui est généralement redouté. 

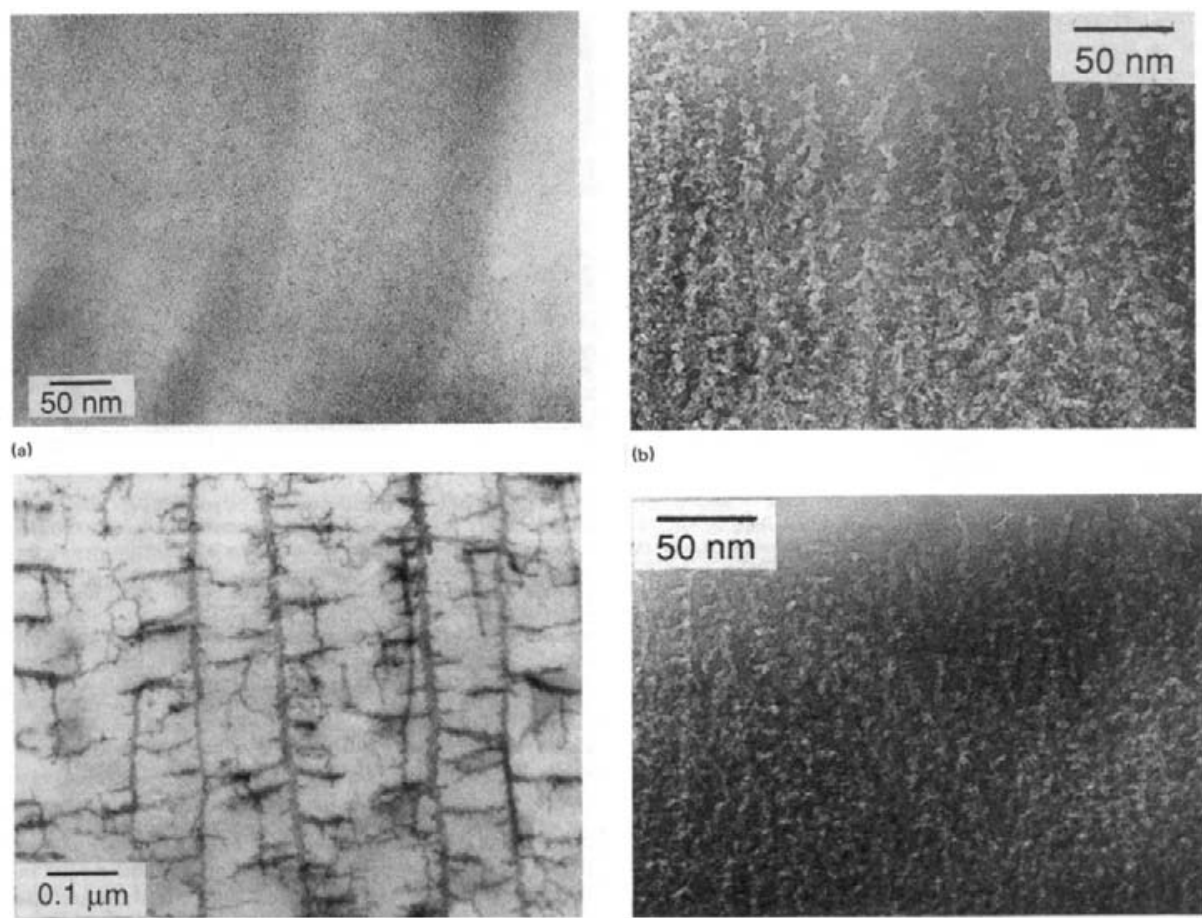

(b)

(c)

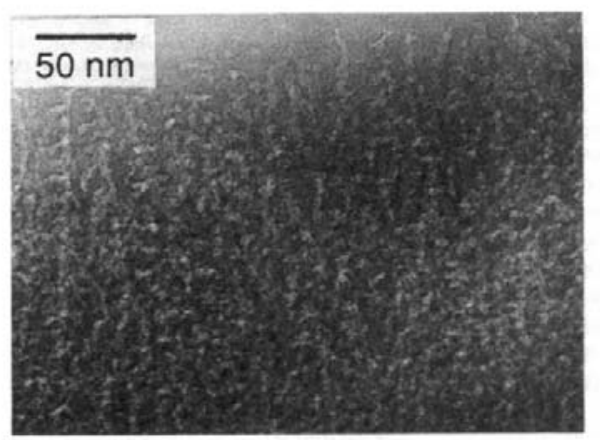

(d)

Figure 7. Clichés de microscopie électronique à transmission montrant les quatre types de silicium poreux : (a) type $p$ : structure en éponge ; (b) type $p^{+}$: structure colonnaire ; (c) type $n$ : macropores; (d) type $n^{+}$: également colonnaire. $\mathrm{D}^{\prime}$ après [40], avec la permission des auteurs.

[Scanning electron micrographs showing four different types of porous silicon: (a) $p$-type: sponge-like structure; (b) $p^{+}$-type: columnar structure; (c) $n$-type: macropores; (d) $n^{+}$-type: columnar as well. From [40], with the permission of the authors.]

- du type $p^{+}\left(\rho \sim 10^{-3} \Omega \mathrm{cm} ; N_{\mathrm{a}} \sim 10^{19} \mathrm{~cm}^{-3}\right)$ : la structure est fortement anisotrope (colonnaire), les pores cylindriques, $\mathrm{d}$ 'une taille de quelques dizaines de nanomètres, et possèdent de nombreux branchement latéraux (Fig. 7b) ;

- du type $n\left(\rho \sim 1 \Omega \mathrm{cm} ; N_{\mathrm{d}} \sim 10^{16} \mathrm{~cm}^{-3}\right)$ : la formation de la couche nécessite un apport de trous par illumination. À faible, épaisseur, la structure est similaire au type $p$. Au-delà d'une centaine de nanomètres, des macropores se forment (Fig. 7c);

- du type $n^{+}\left(\rho \sim 10^{-3} \Omega \mathrm{cm} ; N_{\mathrm{d}} \sim 10^{19} \mathrm{~cm}^{-3}\right)$ : la structure est assez semblable à celle du type $p^{+}$(Fig. $7 \mathrm{~d}$ ).

Les deux premiers types sont de loin les plus étudiés. Pour notre part, nous nous limiterons au cas du type $p$ nanoporeux, qui possède la propriété de luminescence. 


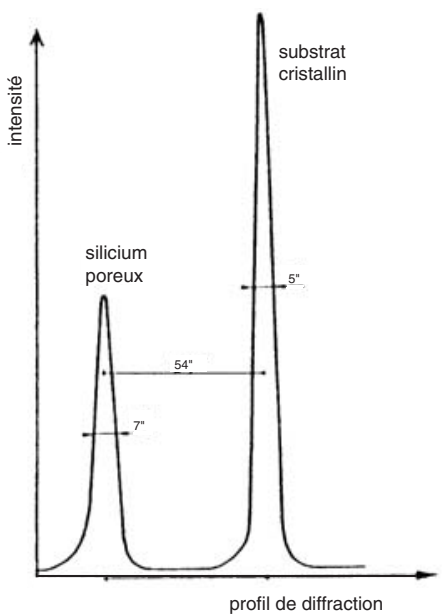

Figure 8. Profil de diffraction de rayons $X$ à haute résolution obtenu sur un échantillon de type $p^{+}$. D'après [43], avec la permission des auteurs.

[High resolution $\mathrm{X}$-ray diffraction profile measured on a $p^{+}$-type porous silicon sample. From [43], with the permission of the authors.]

\section{Cristallinité}

Le silicium poreux conserve après sa formation une cristallinité inattendue, proche de celle du substrat de départ.

Le profil historique de diffraction de rayons $\mathrm{X}$ à haute résolution, obtenu sur un échantillon de type $p^{+}$, est reproduit sur la figure 8 . Les largeurs des pics dus au silicium poreux et au substrat cristallin sont comparables. L'écart angulaire entre les pics indique une légère élongation $\left(10^{-4}\right)$ du paramètre de maille du réseau cristallin de la couche poreuse, dans une direction normale à la surface, attribué aux contraintes liées à la présence de liaisons $\mathrm{Si}-\mathrm{H}$ [43]. À noter que dans le cas du type $p$, la qualité cristalline est légèrement inférieure, et on mesure des dilatations de $l^{\prime}$ ordre de $10^{-3}$.

\subsection{Propriétés optiques}

\section{Luminescence}

La photoluminescence à température ambiante, découverte par Canham en 1990 [37], a déclenché l'intérêt que l'on connaît pour le silicium poreux. La figure 9, tirée de l'article original, illustre l'émission rouge, donc à des énergies plus importantes que le gap du silicium massif $(1,1 \mathrm{eV})$, mesurée sur des échantillons de forte porosité.

Dès sa découverte, la luminescence a suscité un grand intérêt, notamment pour tenter d'expliquer son existence. Pas moins d'une vingtaine de modèles ont été d'abord proposés. Aujourd'hui, bien qu'aucun de ceux qui sont encore d'actualité ( $c f$. la revue de Cullis et al. [29]) n'ait apporté de réponse irréfutable, c'est celui du confinement quantique, qui attribue la photoluminescence rouge ${ }^{3}$ au cristallites de silicium confinées qui est le plus souvent évoqué. Dans ce modèle (le premier

3. D'autres photoluminescences ont été mises en évidence, dans le bleu et dans le proche infrarouge. D'efficacité moindre, elles sont attribuées à des effets radicalement différents [28]. 


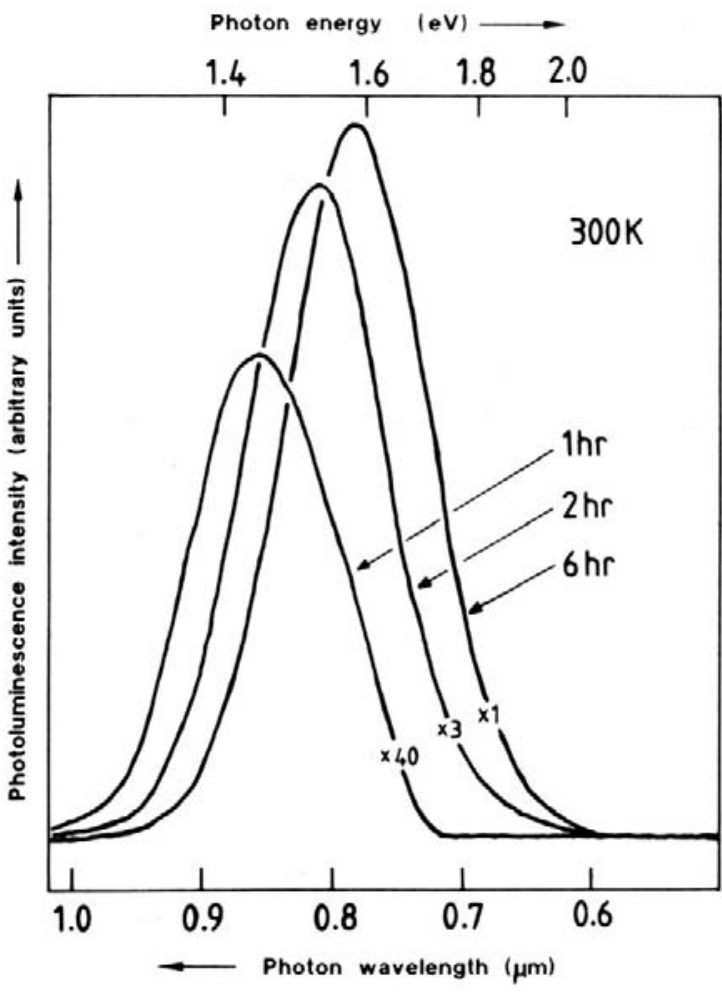

Figure 9. Spectre de photoluminescence à température ambiante tiré de l'article original de Canham [37] (avec la permission de l'auteur). Il s'agit d'un échantillon de type $p$, de porosité initiale $70 \%$, qui a séjourné après son anodisation pendant les durées indiquées dans une solution d'HF. On notera l'augmentation de l'intensité de luminescence ainsi que son décalage vers le bleu.

[Room-temperature photoluminescence spectrum, from the original paper by Canham [37] (with the permission of the author). The $p$-type sample, of initial porosity $70 \%$ was kept in a HF solution, during the times indicated close to each plot. Note the increase of luminescence intensity and the blue shift.]

suggéré $[37,38])$, le confinement quantique augmente l'énergie des porteurs, d'où le décalage énergétique. En outre, la localisation des porteur, conjuguée à la passivation de la surface des cristallite par des liaisons $\mathrm{Si}-\mathrm{H}$ ou $\mathrm{Si}-\mathrm{O}$ augmente de façon drastique la part relative des recombinaisons radiatives [44].

Une autre approche, faisant intervenir des composés luminescents à la surface des cristallites, a encore de nombreux partisans, elle bute cependant sur l'interprétation de nombreux résultats expérimentaux.

L'électroluminescence a également été mise évidence, lors de l'oxydation anodique du silicium poreux [45]. Malgré les progrès accomplis pour créer des contacts solides (métaux, polymères), les diodes réalisées restent handicapées par une faible efficacité et une durée de vie limitée, et leur utilisation en série paraît aujourd'hui sérieusement compromise [29].

Quoi qu'il en soit, rien n'a encore permis de trancher quant à la nature homogène (chaque émetteur posséderait une émission large) ou inhomogène (distribution d'émetteurs monochromatiques) de la raie d'émission du silicium poreux, même si on peut penser que l'émission est certainement inhomogène, dans le mesure où l'énergie d'excitation joue un rôle sélectif [46]. 


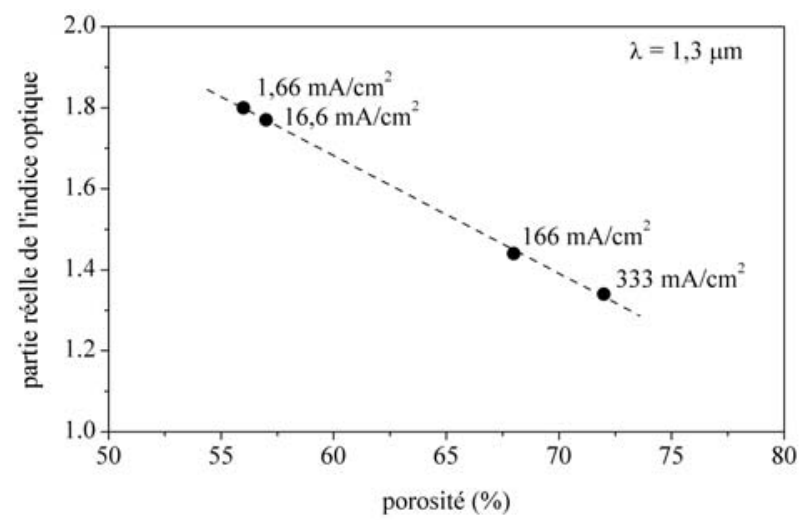

Figure 10. Variation de la partie réelle de l'indice du silicium poreux en fonction de la porosité de la couche. Valeurs mesurées à $\lambda=1,3 \mu \mathrm{m}$ sur des échantillons de type $p$, anodisés dans une solution contenant $35 \%$ de HF. Les densités de courant correspondantes sont indiquées en face de chaque point. La plage des indices accessibles (de 1,3 à 1,8 ici) est propre aux conditions expérimentales.

[Variation of the real part of the optical index of porous silicon versus porosity. Values measured at $\lambda=1.3 \mu \mathrm{m}$ on $p$-type samples, anodized in a $35 \% \mathrm{HF}$ solution. The corresponding current densities are indicated close to each datapoint. The accessible index range (here from 1.3 up to 1.8) depends on the experimental conditions.]

\section{Indice optique}

L'indice optique du silicium poreux a été étudié bien avant sa luminescence. Dès 1984, Pickering et al. [47] mettent en évidence que le silicium nanoporeux peut être décrit dans la gamme des longueurs d'ondes optiques par un indice optique moyen, en raison de la taille nanométrique des hétérogénéités qui le constituent.

L'indice du silicium poreux est un compromis entre celui du silicium massif $(\operatorname{Re}(n) \approx 3,5)$ et celui de l'air $(\operatorname{Re}(n)=1)$, une augmentation de la porosité se traduisant par une diminution de l'indice, comme l'illustre la figure 10. Néanmoins, il serait vain de vouloir prédire avec précision l'indice d'une couche poreuse connaissant sa porosité ; de nombreux modèles plus ou moins élaborés (LorentzLorenz, Maxwell-Garnet, etc.) existent pour décrire ce genre de milieux hétérogènes, mais leurs résultats assez décevants dans le cas du silicium poreux [48] illustrent la complexité du matériau.

Il est en outre apparu, lors de mesures précises en géométrie guidée, que le silicium poreux était un matériau anisotrope uniaxe positif (l'indice extraordinaire $n_{\mathrm{e}}$ est supérieur à l'indice ordinaire $n_{\mathrm{o}}$ ), avec un axe optique perpendiculaire à la surface du substrat [49]. Le paramètre d'anisotropie $\delta$, défini par

$$
\delta=\left(n_{\mathrm{e}} / n_{\mathrm{o}}\right)^{2}-1
$$


prend dans le cas du silicium poreux de type $p$, pourtant catalogué comme isotrope $^{4}$, des valeurs de l'ordre de 0,05 . La polarisation de la photoluminescence qui en résulte a été attribuée à la forme ellipsoïdale des cristallites, avec une orientation préférentielle du grand axe perpendiculairement au plan [50,51].

Quant à la partie imaginaire de l'indice, ses valeurs indiquent des propriétés d'absorption proches de celles du silicium massif : comportement relativement métallique à grande énergie (au-delà du vert), et plutôt diélectrique dans le proche infrarouge.

La gamme intermédiaire (entre $1 \mathrm{et} 2 \mathrm{eV}$ ) a été très étudiée, en raison des réponses qu'elle pouvait apporter quant à l'origine de la luminescence [30]. On s'attendait notamment à observer un décalage du seuil d'absorption vers les hautes énergies, signature de l'augmentation (par confinement quantique) de la valeur du gap. Différentes études ont été menées, par mesures de transmission sur des couches auto-supportées [52], d'effet mirage [53], ou de photoconductivité [54], mais l'interprétation des mesures se heurte à la distribution de taille des cristallites (2-5 nm dans le cas du type $p$ ) : l'allure des spectres de transmission est dominée par l'influence des grosses cristallites, alors que les petites cristallites donnent la forme des spectres de photoluminescence.

\section{Méthodes de structuration}

Nous décrivons dans cette partie les différents moyens qui permettent de structurer le matériau à une échelle inférieure au micron, notamment de façon périodique afin de générer une bande interdite photonique pour les longueurs d'onde optiques.

\subsection{Structuration en profondeur}

\section{Principe}

La structuration en profondeur découle directement de la remarquable propriété décrite en $\S 1.4$. : lors de la formation de la couche, la porosité - et a fortiori l'indice - dépend principalement de la densité de courant qui circule. Il est alors facile de faire varier la porosité en profondeur en modifiant la densité de courant pendant l'anodisation. Comme l'illustre la figure 11, une succession de deux densités de courant différentes aboutit à l'anodisation successive de deux couches distinctes. À noter que la vitesse d'attaque augmente généralement avec le courant utilisé.

Une séquence périodique de courants permet donc de réaliser des multicouches périodiques à base de silicium, possédant des propriétés de bande interdite photonique (réflecteurs de Bragg [55] et microcavités [56]).

4. Dans le cas du silicium poreux de type $p^{+}$, le paramètre d'anisotropie est typiquement deux fois plus important [49]. 


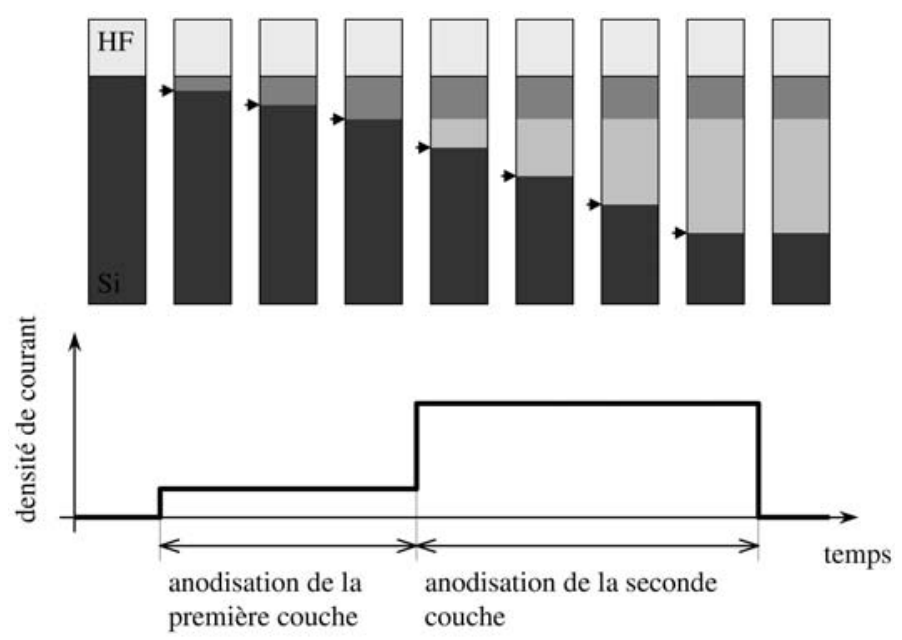

Figure 11. Illustration de la structuration en profondeur. La séquence de courants d'anodisation (en bas) forme successivement deux couches de porosité, donc d'indice différent. La progression de l'attaque aux instants correspondants est également schématisée (en haut). L'interface où se produit l'anodisation est indiquée par une flèche.

[Scheme of the structuration in depth. The sequence of current densities (bottom) etches successively different porosities, i.e., different optical indices. The successive steps of etching are also illustrated (top), and the arrow emphasizes the interface where the anodization takes place.]

On retiendra donc qu'il s'agit d'une formation en profondeur (c'est la couche la plus enterrée qui est formée la dernière), à opposer aux méthodes de croissance (dépôt, épitaxie, etc.).

\section{Limitations}

Nous l'avons vu en $\S 1.4$., les couches sont supposées homogènes en profondeur, et l'épaisseur proportionnelle à la durée d'anodisation. Les structures multicouches ayant une réponse optique très critique, elles constituent des configurations très exigeantes, et ces hypothèses trouvent leurs limites, notamment en raison de la finesse des couches.

En particulier, nous avons constaté lors de l'étude de multicouches que les indices et épaisseurs étaient légèrement différentes de celles qu'on aurait pu déduire des mesures sur des couches épaisses. De tels écarts ont été mis en évidence en étudiant la dispersion du mode d'une cavité luminescente, au moyen de mesures de photoluminescence résolues en angle, au moyen du montage qui sera détaillé en $\S 2.2$., chapitre 4 : les calculs, effectués en toute généralité, montrent en effet que si les couches fines qui constituent les couches du miroir de Bragg ont 
rigoureusement la même épaisseur optique ${ }^{5}$, alors le mode d'une cavité demionde a une énergie indépendante de sa polarisation, même à incidence non nulle (cf. la discussion sur la longueur d'onde de résonance des modes, page 52). De telles considérations nous ont permis de contrôler et de corriger les écarts [14].

\subsection{Structuration dans le plan}

\section{Principe}

Nous avons vu en $\S 1.2$. que l'anodisation d'un substrat de silicium en contact avec une solution d'acide fluorhydrique était conditionnée par la présence de trous. Elle ne nécessite cependant pas obligatoirement une circulation de courant, si l'on utilise par exemple des trous générés en illuminant le matériau [57,58].

Ce procédé est également applicable à partir de silicium massif, ou de couches de silicium poreux déjà formées lorsqu'elles sont encore dans leur électrolyte. L'illumination induit de façon localisée dans le plan une augmentation de la porosité, éventuellement jusqu'à l'élimination totale du silicium. Des réseaux de diffraction ont ainsi pu être gravés en balayant un faisceau focalisé sur une couche de silicium poreux [59]. Pour notre part, nous préférerons utiliser une structuration dite holographique, qui consiste à illuminer le matériau par une figure d'interférence, produite au moyen du montage qui sera décrit en $\S 1$ 1.3., chapitre 4, cette méthode étant beaucoup plus adaptée à la structuration périodique et régulière de grandes surfaces, jusqu'à des pas inférieurs au micron [15].

Excepté le type $p^{+}$, moins sensible, étant donné son fort dopage, aux trous générés optiquement, tous les types de silicium poreux se prêtent à la structuration holographique [60]. Nous nous sommes limité dans ce travail au cas du silicium de type $p$, qui permet de scinder l'élaboration en deux étapes : gravure des couches en profondeur, puis la structuration holographique.

\section{Limitations}

La principale limitation est liée au processus d'absorption de lumière responsable de la création des trous : la lumière, qui provient du dessus de la couche, a donc une action restreinte en surface, sur une certaine épaisseur. Les études précédentes, effectuées sur des structures de grande période, ont montré que la profondeur efficace de structuration était du même ordre que la longueur de pénétration de la lumière utilisée pour l'illumination [60], soit de l'ordre de deux microns, si l'on utilise la raie verte du laser à argon.

\subsection{Croissance contrôlée de macropores}

Le procédé mis au point par Grüning et al. [61] constitue un autre type de structuration particulièrement efficace, basé sur un contrôle de la nucléation et de la croissance des macropores obtenus sur un substrat de type $n$.

5. Ceci ne peut être vrai qu'à un seul angle. 


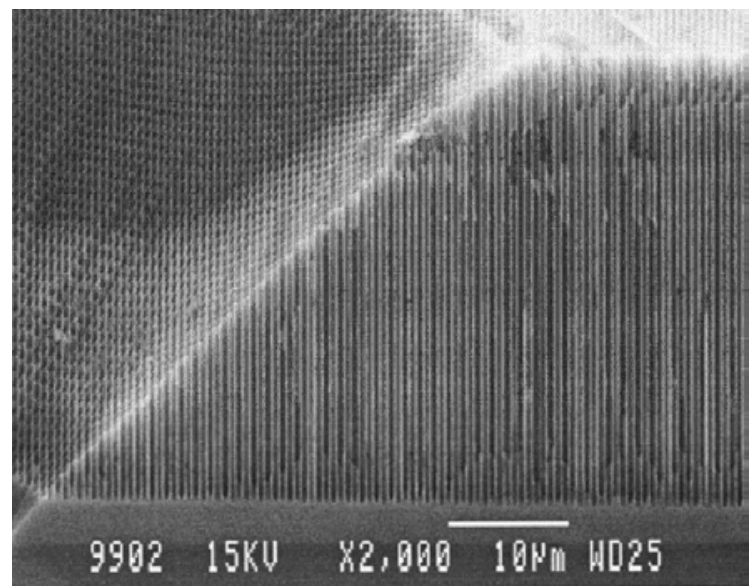

Figure 12. Cliché de microscopie électronique d'une structure de silicium macroporeux. Les pores ont été initiés par lithographie holographique dans un réseau triangulaire de $680 \mathrm{~nm}$ de période. Les pores cylindriques atteignent une profondeur de $50 \mu \mathrm{m}$. D'après [62] (tous droits réservés).

[Scanning electron micrograph of a macroporous silicon structure. Pores have been initiated by holographic lithography and are ordered in a triangular lattice of period $680 \mathrm{~nm}$. The depth of pores can be as large as $50 \mu \mathrm{m}$. From [62] (all rights reserved).]

Le substrat de départ est gravé par lithographie, de telle sorte qu'un réseau d'amorces (sous forme de pyramides inversées) soit créé en surface. Un contrôle fin des paramètres d'anodisation permet de restreindre la nucléation des macropores à ces amorces, puis de poursuivre la croissance des pores dans des conditions de courant et de tension constante (par asservissement $d^{\prime}$ une illumination en face arrière), de telle sorte que le diamètre ne change pas en cours d'anodisation.

En utilisant un procédé de lithographie holographique, la période des motifs a pu être abaissée sous la barre du micron [62], comme l'illustre la figure 12 tout en bénéficiant d'un rapport d'aspect et d'une qualité optique qui ont permis de mettre en évidence une bande interdite bidimensionnelle complète dans le proche infrarouge $(1,55 \mu \mathrm{m})$.

L'intérêt de ces structures réside cependant davantage dans le caractère idéal de leur géométrie bidimensionnelle qui se prête parfaitement à la confrontation avec les prévisions théoriques, que dans leurs applications potentielles, en raison de l'absence de confinement vertical.

Dans ce chapitre, nous avons donné une présentation volontairement large du silicium poreux. Pour la suite de cet ouvrage, basé sur du silicium nanoporeux de type $p$, nous retiendrons qu'il s'agit d'un matériau luminescent, homogène, dont l'indice peut prendre à volonté des valeurs comprises entre 1,2 et 1,8, et dont la transparence l'apparente à un matériau diélectrique dans le proche infrarouge. Nous avons vu en outre que ce matériau se prêtait à une structuration en profondeur ou dans le plan. 


\section{Méthodes expérimentales}

Nous détaillons dans ce chapitre les procédés expérimentaux utilisés à la fois pour la réalisation des échantillons (anodisation et structuration holographique) et pour les caractérisations optiques. Un accent tout particulier est mis sur la configuration expérimentale utilisée pour les caractérisations en optique guidée.

\section{1. Élaboration des échantillons}

\subsection{Préparation du substrat}

Nous avons utilisé des substrats de silicium monocristallin de type $p$, faiblement dopés au bore, de résistivité comprise entre 3 et $7 \Omega \mathrm{cm}$ et d'orientation cristallographique (100). Afin de permettre un bon contact ohmique en face arrière pour l'anodisation, une couche d'aluminium est évaporée à l'arrière du substrat; les opérations de nettoyage, évaporation et recuit sont effectués en salle blanche.

Nous avons occasionnellement utilisé des substrats de faible épaisseur (typiquement $100 \mu \mathrm{m})$ car ils permettent un clivage franc, seul garant de la qualité optique nécessaire pour mener à bien les mesures par la tranche, en géométrie guidée.

\subsection{Anodisation}

La solution électrolytique utilisée pour préparer les échantillons présentés dans ce manuscrit est un mélange d'acide fluorhydrique, d'eau et d'éthanol absolu, dans des proportions volumiques 35:35:30. La présence d'éthanol accroît le mouillage sur le silicium, tout en favorisant le dégagement de l'hydrogène gazeux généré par la réaction chimique.

L'anodisation est réalisée dans la cellule représentée schématiquement sur la figure 13. Les matériaux et la conception satisfont au cahier des charges particulièrement contraignant imposé par la nature hautement corrosive, volatile et toxique de la solution.

Aussi, la cellule est une enceinte fermée, usinée en polyéthylène (cestilene), d'une capacité de quelques millilitres d'électrolyte. Le substrat de silicium est plaqué verticalement contre un orifice circulaire, offrant une surface de $0,9 \mathrm{~cm}^{2}$ 


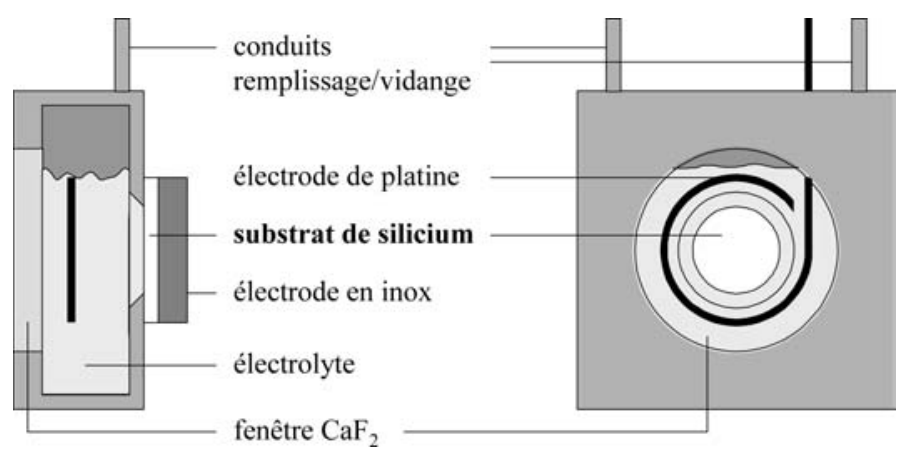

Figure 13. Cellule utilisée pour l'électrolyse. Les joints toriques, sur lesquels s'appuient la fenêtre et le substrat de silicium ne sont pas représentés.

[Electrochemical cell. O-rings (against the window and the silicon substrate) are not represented.]

au contact de l'électrolyte. Remplissage et vidange sont réalisés à travers deux conduits sur le dessus.

Pour permettre une illumination pendant les étapes de photodissolution, une ouverture circulaire en face avant de la cellule est équipée d'une fenêtre en fluorine $\left(\mathrm{CaF}_{2}\right)$, matériau inerte vis-à-vis de l'électrolyte. Ce composant répond en outre aux exigences de l'holographie, telles qu'elles sont décrites en $\S 1.3$.

Le contact dans l'électrolyte est pris au moyen d'un fil de platine rigide, et c'est une électrode en inox, appliquée contre la face arrière du substrat, qui permet le contact arrière. Le courant circule entre ces deux électrodes. Il est fourni par un galvanostat EG\&G PAR 273. Lors de l'anodisation de multicouches, le séquençage des courants est programmée par l'intermédiaire d'un micro-ordinateur. Les densités de courants $j$ utilisées sont typiquement comprises entre 10 et $400 \mathrm{~mA} / \mathrm{cm}^{2}$.

Après l'anodisation, l'électrolyte est vidangé et le rinçage est assuré par une circulation d'eau désionisée pendant cinq minutes. Lorsque la structure formée possède des zones de forte porosité, ce rinçage est suivi d'un rinçage à l'éthanol, puis au pentane. Cette méthode, proposée par Belmont et al. [63], permet d'accroître la stabilité mécanique des couches en diminuant les contraintes lors du séchage, qui est ensuite effectué sous flux d'azote.

La disposition verticale du substrat, assez peu courante, est nécessaire : elle permet de s'affranchir de toute perturbation de l'illumination frontale par l'interface agitée de l'électrolyte; en outre, les bulles d'hydrogène dégagées sont alors chassées hors du trajet du faisceau. Mais elle n'est pas sans inconvénient : nous avons en effet constaté que les anodisations pratiquées à la verticale donnaient systématiquement des échantillons peu homogènes. La raison de ce phénomène n'est pas clairement établie. Cependant, dans la mesure où les bulles générées par la réaction chimique s'évacuent verticalement, on peut craindre qu'elles induisent une convection de la solution devant et dans la couche poreuse, pouvant créer des conditions chimiques variables en différents points de l'échantillon. 


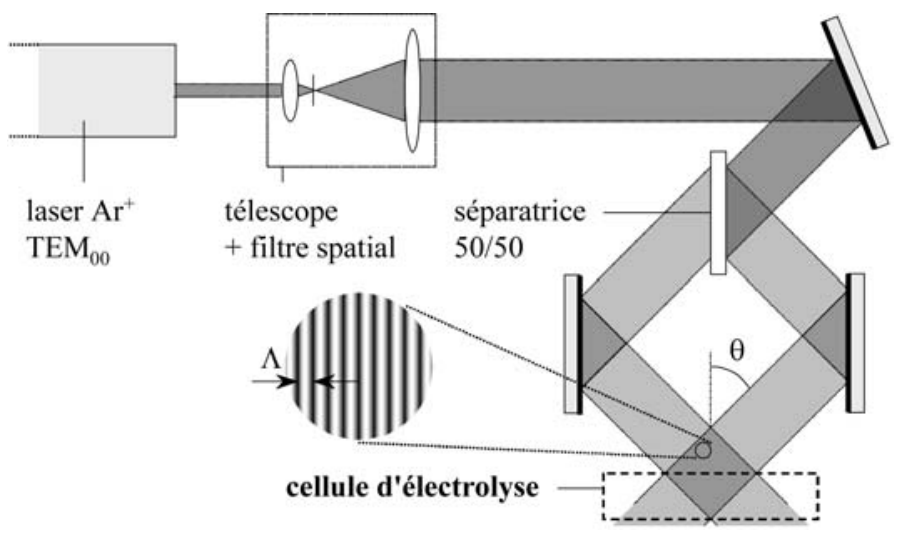

Figure 14. Schéma du montage utilisé pour la structuration holographique. Tous les faisceaux sont dans un plan horizontal. La polarisation est verticale. Les deux derniers miroirs de renvoi sont orientables afin de pouvoir ajuster l'angle $\theta$. La cellule d'électrolyse (en pointillés) est placé dans une zone où les faisceaux interfèrent.

[Experimental setup used for the holographic structuration. All beams are in the horizontal plane. The polarization is vertical. The angle $\theta$ can be easily changed by rotating the two last mirrors. The electrochemical cell (plotted in dotted line) takes place in the region where the beams interfere.]

Pour s'affranchir partiellement de ce problème, l'anodisation dans le noir est faite en maintenant la cellule à l'horizontale ; la cellule n'est redressée qu'au moment de la structuration holographique.

\subsection{Structuration holographique}

Le principe de la structuration holographique repose sur l'illumination de l'échantillon par une figure d'interférence. En particulier, la superposition de deux ondes planes cohérentes polarisées TE, de longueur d'onde $\lambda$, en vis-à-vis avec un angle $\mathrm{d}^{\prime}$ incidence $\theta$, donne lieu à des franges non localisées (plans parallèles d'égale intensité), comme l'illustre la figure 14 : l'intensité lumineuse est modulée sinusoïdalement avec une période

$$
\Lambda=\lambda /(2 \sin \theta) .
$$

La cellule électrolytique, représentée en pointillés, est placée dans cette distribution d'intensité, qui s'en trouve peu changée. En effet, si la réfraction dans les différents milieux traversés (fenêtre, électrolyte) modifie l'angle d'incidence des faisceaux, la période du motif, elle, n'est pas affectée, car le facteur $\sin \theta$ de l'équation (4.1) doit être remplacé dans un milieu d'indice $n_{i}$ par $n_{i} \sin \theta_{i}$ qui a la même valeur, selon les lois de Snell-Descartes ${ }^{1}$. En outre, si en toute rigueur les réflexions

1. Ceci n'est vrai que parce que les faisceaux sont symétriques par rapport à un plan perpendiculaire aux interfaces. 
sur les interfaces de la cellule produisent également des franges parallèles à ces interfaces, les coefficients de réflexion peu élevés contre l'échantillon donnent des franges peu contrastées, qui se décalent continûment au cours de la dissolution, si bien que leur intensité est uniformisée. Finalement, le seul effet notable est la chute d'intensité en profondeur de la couche, causée par l'absorption du faisceau par le matériau.

Le montage expérimental, schématisé sur la figure 14, utilise comme source lumineuse le mode TEM $_{00}$ de la raie verte $(\lambda=514,5 \mathrm{~nm})$ d'un laser Coherent Innova 90-6 à argon ionisé. Pour obtenir une figure d'interférence la plus proche du cas idéal qui vient d'être exposé, il convient de respecter les règles suivantes :

- les faisceaux doivent être assimilables à des ondes planes. L'expansion du faisceau gaussien au moyen d'un télescope permet de réduire le rayon de courbure du front d'onde, tout en produisant un étalement de la distribution transversale d'intensité. Dans notre cas, un grandissement de vingt fois produit un faisceau de diamètre $30 \mathrm{~mm}$, réduit à $20 \mathrm{~mm}$ par un diaphragme. En outre, nous veillons à n'utiliser que des composants optiques dont la planéité est garantie meilleure que $\lambda / 10$;

- les deux faisceaux doivent être cohérents. Ils sont obtenus dans notre montage par division d'amplitude, au moyen d'une lame séparatrice. Dans le montage tel qu'il est représenté, les longueurs des parcours des deux faisceaux sont quasiment identiques, et la surface à illuminer est de l'ordre du centimètre carré, si bien que la longueur de cohérence de quelques centimètres est suffisante. Pour un montage moins symétrique (avec plus de deux faisceaux, par exemple), ou pour illuminer de plus grandes surfaces, il sera nécessaire d'utiliser un étalon intra-cavité, afin de sélectionner une seule fréquence, et d'obtenir ainsi une longueur de cohérence décuplée ;

- le faisceau doit être exempt de tout défaut d'intensité, comme les franges, trous, ou anneaux, dus à la diffusion par des rayures, poussières, etc. L'utilisation d'un diaphragme en diamant ${ }^{2}$, de diamètre $10 \mu \mathrm{m}$ au foyer de la première lentille du télescope permet un filtrage spatial, en éliminant la contribution des défauts, qui est alors défocalisée hors de l'axe du faisceau. La propreté des composants est soigneusement contrôlée. Ils bénéficient généralement d'un traitement antireflet (excepté la fenêtre de la cellule, pour des raisons de compatibilité chimique avec l'électrolyte). Les composants plans (séparatrice et fenêtre) utilisent en outre des substrats en coin $\left(1^{\circ}\right)$, afin de dévier hors de l'axe initial du faisceau les réflexion sur la deuxième interface ;

- le déphasage entre les deux faisceaux doit être maintenu rigoureusement constant — à l'échelle de la longueur d'onde — pendant la durée de l'illumination. La fixation solide sur une table en marbre, montée sur amortisseurs, assure à l'ensemble du montage la rigidité et la stabilité nécessaires. La boîte à gants qui surmonte cette partie du montage le protège à la fois des poussières et des gradients thermiques.

2. Il s'agit en réalité d'une filière, fournie gracieusement par Patrick Alleysson, Société des Filières Balloffet, 01150 Lagnieu, France. 
Les échantillons sont typiquement illuminés pendant quelques minutes par deux faisceaux d'une densité de puissance de quelques milliwatts par centimètre carré.

Le principal avantage de cette méthode réside dans sa simplicité : la structuration ne nécessite pas de masque, la période du motif est facilement modifiable en changeant l'angle $\theta$, et un montage soigné permet de garantir une homogénéité parfaite sur de grandes surfaces. Une configuration plus complexe, utilisant davantage de faisceaux, permet d'envisager des motifs plus complexes, difficilement réalisables avec un masque. La limite inférieure de la période, d'après l'équation (4.1), est $\lambda / 2$ pour des faisceaux rasants. En pratique, l'ouverture angulaire maximale de la fenêtre nous limitera à une valeur légèrement supérieure ${ }^{3}$.

\subsection{Oxydation anodique}

L'oxydation anodique est réalisée après rinçage dans la même cellule électrochimique, en utilisant une solution molaire d'acide chlorhydrique, et une densité de courant constante de $1 \mathrm{~mA} / \mathrm{cm}^{2}$. La circulation de courant est maintenue jusqu'à ce que le contact entre la couche de silicium poreux et le substrat soit coupé [64]. Ceci se manifeste par une brusque envolée de la tension à appliquer, et nous choisissons de couper à la valeur de $10 \mathrm{~V}$.

\section{Caractérisations optiques par la surface}

\subsection{Mesures de réflectivité}

La réflectivité des échantillons a été mesurée perpendiculairement à leur surface, de l'ultraviolet au proche infrarouge $(0,250-2,5 \mu \mathrm{m})$, au moyen d'un spectrophotomètre double faisceau Perkin-Elmer Lambda 9 équipé d'un accessoire de réflectivité absolue. Un spectre typique, faisant apparaître les franges d'interférence dues à l'épaisseur finie de la couche, est tracé sur la figure 15a. Ces mesures sont effectuées de façon quasi-systématique, car elles permettent une caractérisation précise et non destructive des couches poreuses.

La méthode que nous utilisons consiste à ajuster sur le spectre expérimental un spectre théorique calculé rigoureusement en utilisant une méthode à base des matrices de transfert évoquée en $\S 2$., chapitre 2. Les principaux paramètres à ajuster sont l'épaisseur et les valeurs d'indice complexe de la couche en plusieurs points dans toute la gamme spectrale étudiée, le programme se chargeant de l'interpolation. Les valeurs d'indice du substrat de silicium sont issues de la littérature [65]. La courbe tracée en pointillés sur la figure 15a illustre la précision d'ajustement réalisé sur un spectre de réflectivité de couche simple. La dispersion des parties réelle et imaginaire de l'indice est également reportée respectivement sur les figures $15 \mathrm{~b}$ et $15 \mathrm{c}$.

3. En réalité, une cellule à fenêtres latérales, perpendiculaires à l'échantillon permettrait d'atteindre la valeur $\lambda / 2 n$, où $n$ est l'indice de l'électrolyte. Nous n'avons pas eu la nécessité de descendre aussi bas. 


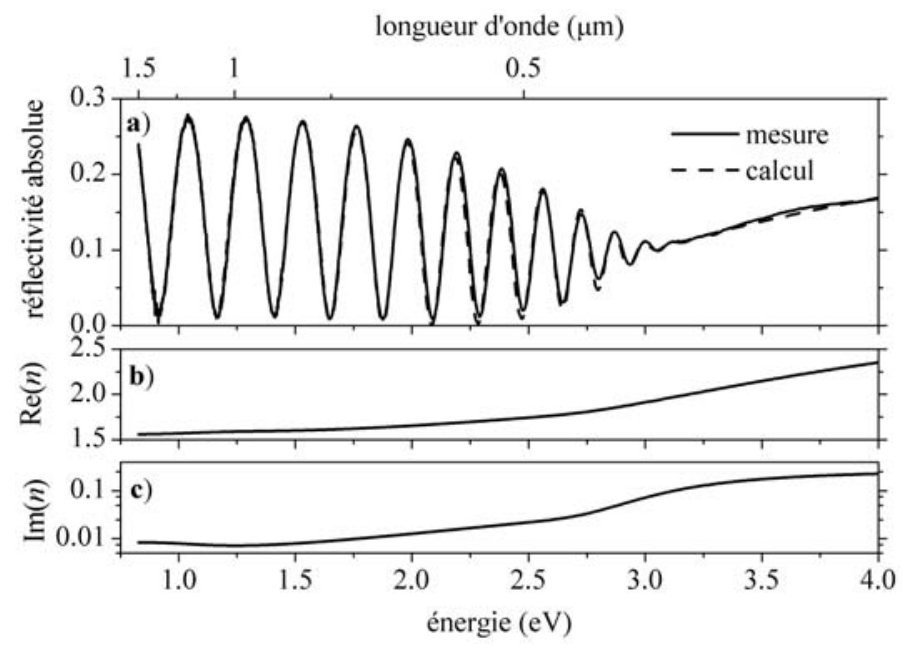

Figure 15. (a) Spectre de réflectivité d'une couche simple de silicium poreux. Le spectre théorique (en pointillés) a été ajusté sur le spectre expérimental (en trait plein). (b) Valeur de la partie réelle et (c) imaginaire de l'indice utilisées dans l'ajustement. Le calcul utilise une épaisseur de couche de 1,52 $\mu \mathrm{m}$.

[(a) Reflectance spectrum of a single porous silicon layer. The theoretical spectrum (dotted line) has been fitted to the experimental spectrum (solid line). (b) Value of the real and (c) imaginary part of the index used for the fit. The calculation considers a layer of thickness $1.52 \mu \mathrm{m}$.]

L'intérêt de cet ajustement réside dans son caractère unique. En effet, si c'est le produit de l'épaisseur par la partie réelle de l'indice qui détermine l'interfrange, ce sont les indices des matériaux (couche et substrat) qui détermineront seuls le contraste et le niveau moyen de réflectivité. En particulier, la zone où les franges disparaissent (au-delà de $3 \mathrm{eV}$ dans notre exemple) à cause de l'absorption qui ne permet plus à la lumière d'atteindre l'interface inférieure de la couche, la réflectivité est simplement déterminée par les lois de Fresnel, à l'interface d'un milieu semi-infini. De telles considérations permettent de se convaincre des rôles indépendants joués par l'épaisseur et l'indice. Avec en outre comme hypothèse que l'indice varie de façon monotone dans la gamme considérée, nous avons pu vérifier qu'un seul jeu de paramètres permettait un ajustement correct.

Cette méthode est également applicable à des structures plus complexes, composées de multicouches, même si l'ajustement d'une courbe calculée est plus délicat, tant le nombre de paramètres intervenant peut s'accroître. Il est également possible de prendre en compte la rugosité que l'on rencontre parfois à l'interface inférieure de certains types de couches [66] (la valeur de la rugosité quadratique moyenne est alors introduite et elle intervient dans les coefficients des matrices de transfert aux interfaces [67]) ainsi que d'éventuelles inhomogénéités d'épaisseur. 


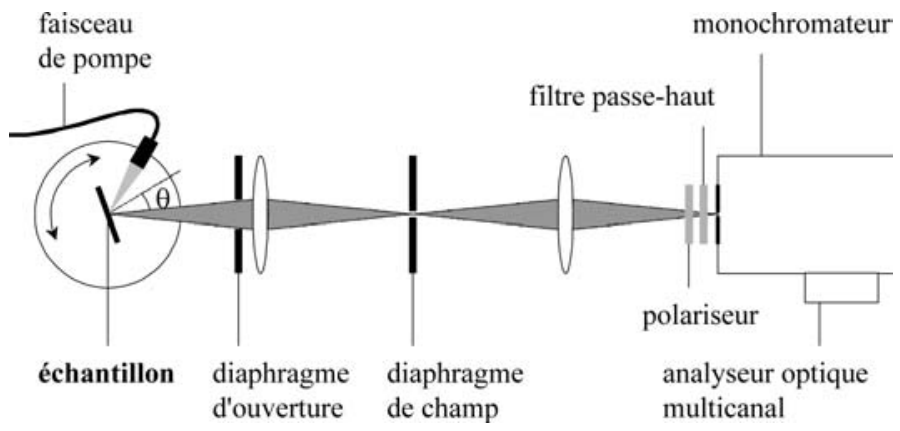

Figure 16. Montage utilisé pour les mesures de photoluminescence résolues en angle. Le faisceau de pompe est fixe par rapport à l'échantillon lorsque l'on modifie l'angle $\theta$.

[Experimental setup used for angle-resolved photoluminescence measurements. The angle of the pumping beam remains unchanged when the angle $\theta$ is modified.]

\subsection{Mesures de photoluminescence}

Pour les mesures de de photoluminescence (PL) émise par la surface des échantillons, nous avons utilisé le montage schématisé sur la figure 16. Son principal intérêt réside dans le contrôle de l'angle et de l'ouverture angulaire de collection, tout en garantissant des conditions de pompage constantes.

L'échantillon est placé sur un support orientable. Le faisceau de pompe, fourni par le laser argon décrit précédemment, est transporté par une fibre optique multimode dont la sortie est solidaire du porte-échantillon, et focalisé sur une tâche d'environ $1 \mathrm{~mm}^{2}$ à la surface de l'échantillon. À noter que le faisceau issu de la fibre multimode a perdu toute polarisation. La tâche lumineuse est d'abord imagée sans grandissement sur un diaphragme de champ, lui-même conjugué de la fente d'entrée du monochromateur. Cette image intermédiaire n'apporte rien à la mesure (la fente du monochromateur est beaucoup plus restrictive), mais doit être vue comme un report dans un plan plus dégagé de la fente du monochromateur peu accessible autrement. Le contrôle de la région analysée est ainsi facilité. Un filtre passe-haut (gélatine Kodak 23A) permet d'éliminer la lumière parasite provenant $\mathrm{du}$ faisceau de pompe, et la polarisation adéquate est sélectionnée par à un film polarisant Polaroid HN 38.

L'analyse spectrale est confiée à un monochromateur Chromex 250IS équipé d'un réseau de 80 traits par millimètre, blazé à $870 \mathrm{~nm}$, offrant une résolution de l'ordre du nanomètre. L'acquisition se fait via un analyseur optique multicanal Princeton 512TKB, basé sur un dispositif à couplage de charges (CCD) en silicium, composé d'une matrice de 512 pixels de côté, refroidi à $-50{ }^{\circ} \mathrm{C}$ par un élément Peltier. Un micro-ordinateur permet de collecter les données.

On utilisera soit la raie verte $(514,5 \mathrm{~nm})$ soit la raie bleue $(457,9 \mathrm{~nm})$ du laser argon. La densité de puissance de pompage est de l'ordre de quelques milliwatts par millimètre carré. On travaillera avec une ouverture angulaire de $1^{\circ}$ de demi-angle 
au sommet. Une accumulation pendant quelques secondes suffit généralement à rendre le rapport signal/bruit acceptable.

\section{Caractérisations optiques en géométrie guidée}

Le principe des mesures en géométrie guidée est relativement simple : il s'agit d'introduire la lumière dans une structure guidante, puis de la collecter en fin de parcours, afin d'en réaliser l'analyse spectrale. Dans la pratique, les difficultés sont liées non seulement à la taille microscopique (au moins le long d'une direction) de l'objet à étudier, mais aussi à l'étendue spectrale de la lumière à utiliser.

Nous détaillons ici un ensemble de configurations qui permettent une étude spectrale de structures guidantes. Nous proposons l'introduction de lumière selon deux procédés : l'injection de lumière blanche par la tranche ou l'excitation d'un émetteur interne. La sélectivité de la collection, ainsi que les méthodes d'acquisition du signal sont ensuite décrites. Enfin, nous discutons les conséquences de la propagation simultanée de plusieurs modes guidés dans la structure.

\subsection{Cahier des charges}

L'absorption du silicium poreux implique d'importantes restrictions du point de vue des longueurs d'ondes envisageables : la longueur des échantillons étant de l'ordre du millimètre, il n'est pas possible de travailler dans le visible. Comme nous souhaitons en outre sonder une grande plage spectrale, nous utiliserons la quasi-totalité du proche infrarouge $(0,7 \mu \mathrm{m}<\lambda<1,6 \mu \mathrm{m})$, ce qui limite fortement l'éventail de détecteurs utilisables.

L'épaisseur des échantillons est moins critique : dans le mesure où les contrastes d'indice accessibles ne sont pas colossaux, il n'est pas utile de descendre au-dessous d'une épaisseur d'un micron pour avoir une structure monomode (ou tout au moins faiblement multimode). L'injection de lumière est délicate, mais tout à fait possible avec une bonne mécanique de précision.

Enfin, signalons que les guides ne sont pas confinés latéralement. Ceci facilite l'injection de la lumière, mais le faisceau guidé qui diverge (dans le plan du guide) doit être entièrement collecté en sortie. Nous verrons par la suite que cette caractéristique géométrique n'est pas contraignante, que notre configuration expérimentale l'utilise avec profit.

\subsection{Injection de lumière blanche}

\section{Description du montage}

Notre source de lumière blanche est une simple ampoule à filament de tungstène : le spectre d'émission, proche de celui du corps noir, couvre une large gamme, du visible à l'infrarouge. 


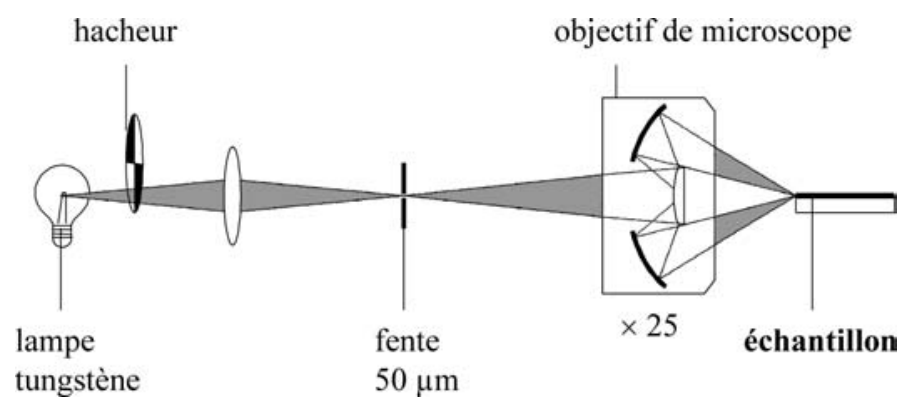

Figure 17. Montage pour l'injection de lumière blanche dans le guide d'onde. Le filament, la fente et la tranche de l'échantillon sont rectilignes et perpendiculaires au plan de la figure, qui n'est pas à l'échelle.

[Experimental setup for the injection of white light in the waveguide. The filament, the slit, and the sample edge are rectilinear and perpendicular to the plane of the figure, which is not to scale.]

Le montage est représenté sur la figure 17. Le filament, qui a été choisi très fin et rectiligne, est parallèle à la tranche horizontale du guide (ces deux objets sont donc perpendiculaires au plan de la figure). L'image du filament est projetée sur une fente également horizontale de hauteur $50 \mu \mathrm{m}$. Ceci constitue une source de dimension réduite, qui est à son tour imagée sur la tranche du guide au moyen d'un objectif de microscope ${ }^{4}$ de grandissement 25 (ouverture numérique 0,4). L'image projetée, longiligne, d'environ $2 \mu \mathrm{m}$ de hauteur, illumine la tranche sur une longueur de $40 \mu \mathrm{m}$. La modulation de l'intensité, réalisée au moyen d'un hacheur, est nécessaire au traitement du signal, comme il en sera discuté en § 3.4.

Comme il est indispensable que le couplage soit réalisé avec une efficacité similaire dans toute la plage spectrale, nous avons utilisé un objectif de microscope de type Cassegrain, que l'utilisation de miroirs métalliques rend rigoureusement achromatique. La grande distance de travail (ici 14,5 mm) est un autre avantage particulièrement appréciable dans notre cas. Quant à l'aveuglement axial dû à l'ombrage créé par le petit miroir convexe, il constitue le seul défaut notable, et nous avons pu nous en accommoder.

\section{Efficacité du couplage}

Malgré la forme peu conventionnelle du faisceau incident, cette configuration permet un couplage efficace dans un guide plan. Expérimentalement, l'observation de la tranche du guide illuminée en sortie montre que la lumière se propage effectivement dans le plan du guide, mais uniquement autour de deux directions obliques symétriques, comme l'illustre la figure 18a.

4. Pour obtenir effectivement le grandissement nominal, la distance standard de $150 \mathrm{~mm}$ a été respectée entre la fente et la bague de l'objectif. 


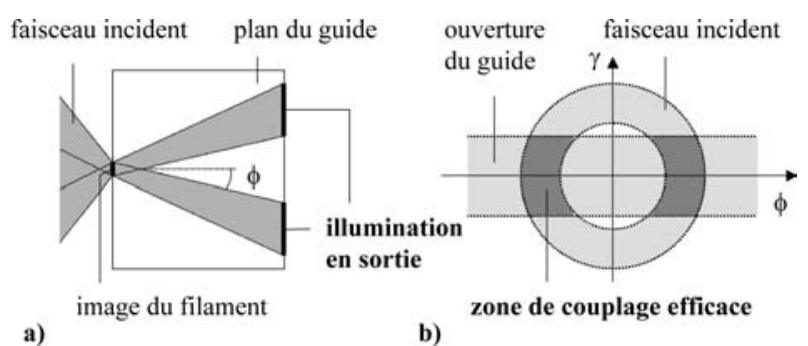

Figure 18. (a) Propagation des faisceaux guidés vue du dessus du guide. (b) Ouvertures respective du faisceau incident et du mode guidé (vue frontale). Le couplage n'est possible que dans les zones où il y a recouvrement.

[(a) Top view of the propagation of the guided beams. (b) Respective apertures of the incident beam and of the guided mode (front view). Coupling is possible in regions of overlapping.]

Comme nous l'avons vu en $\S 3.5$., chapitre 2 , le couplage de la lumière par la tranche est un phénomène complexe. On peut cependant expliquer qualitativement l'effet observé en considérant à la fois la géométrie du faisceau incident, compris entre deux cônes coaxiaux, et l'ouverture angulaire du mode guidé, restreinte verticalement. La figure $18 \mathrm{~b}$ illustre les deux géométries, où nous avons noté $\phi$ l'azimut (dans le plan du guide) et $\gamma$ la déclinaison (angle vertical). Le couplage à l'intérieur du guide n'est possible que dans la zone de recouvrement des deux ouvertures. Une ouverture de mode telle que celle dessinée permet d'envisager un couplage limité à des valeurs d'azimut non nulles et symétriques. Aucune lumière ne peut se propager à azimut nul, car les trop fortes déclinaisons imposées par l'ouverture de l'objectif sont alors hors de l'ouverture du mode.

Expérimentalement, nous avons constaté des efficacités globales de couplagecollection tout à fait raisonnables, de l'ordre de la dizaine de pour cent.

L'injection de lumière blanche permet d'exploiter pleinement la large gamme spectrale offerte par un filament de tungstène. Cependant, l'injection par la tranche n'est pas sans inconvénient. La principale limitation est d'ordre pratique : la qualité des interfaces qui est requise est difficile à obtenir, et c'est pour cette raison qu'il est difficile d'obtenir par clivage des échantillons de longueur inférieure au millimètre. $\mathrm{D}^{\prime}$ un point de vue mécanique, le couplage réclame en outre une extrême précision. Le principal risque réside dans des conditions de couplage peu reproductibles et parfois peu stables dans le temps.

\section{3. Émetteur interne}

L'utilisation d'un émetteur intégré à la structure à étudié est une méthode qui a fait ses preuves pour la caractérisation de structures guidantes à bande interdite photonique [68]. Elle permet de s'affranchir de l'injection de lumière qui peut être problématique, notamment lorsque l'épaisseur des couches guidantes est très faible. 


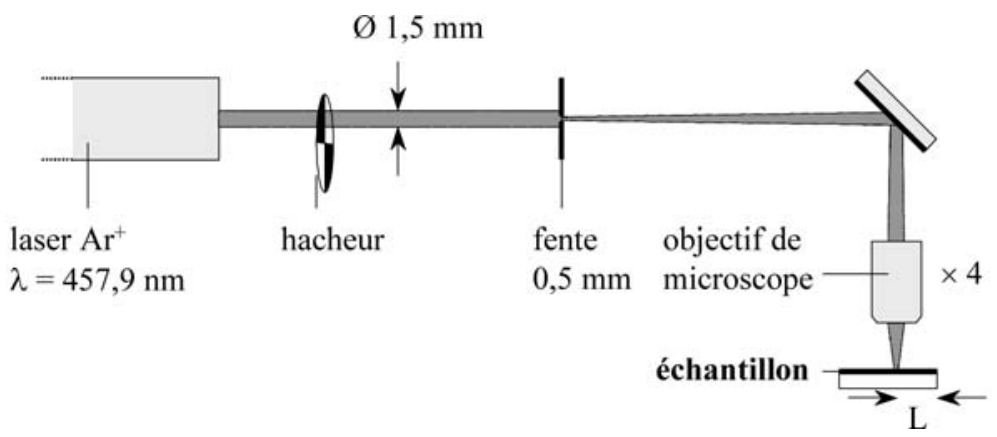

Figure 19. Montage utilisé pour un pompage localisé d'émetteurs intégrés à la structure. [Experimental setup used for a localized pumping of emitters integrated within the structure.]

Il s'agit donc d'utiliser une structure guidante qui possède en outre des propriétés de photoluminescence. L'émetteur peut être le matériau lui-même, comme dans notre cas, mais l'insertion d'un autre système (molécules de colorant, puits ou boîtes quantiques) est également possible. En sélectionnant spatialement une zone au moyen du faisceau de pompe, on crée une source localisée. Si la densité d'émetteurs est constante dans le plan, déplacer le faisceau de pompe revient à déplacer virtuellement l'émetteur.

Nous utilisons le montage qui est présenté sur la figure 19. La pompe est la raie bleue $(\lambda=457,9 \mathrm{~nm}) \mathrm{d}$ 'un laser à argon. Cette longueur d'onde permet un pompage efficace et superficiel en raison du fort coefficient d'absorption du silicium poreux. Le faisceau, modulé par un hacheur, est envoyé sur une fente horizontale de $500 \mu \mathrm{m}$. L'image de cette fente est ensuite projetée au moyen d'un objectif de microscope standard de faible grandissement sur la surface de l'échantillon. On obtient ainsi une illumination de forme grosso modo rectangulaire, de dimensions $130 \mu \mathrm{m} \times 400 \mu \mathrm{m}$, la dimension la plus longue étant parallèle à la tranche de sortie, à une distance $L$.

La méthode consistant à focaliser directement le faisceau parallèle du laser n'a pas été retenue, car les densités de puissance obtenues causaient au matériau des dégâts irréversibles. Avec notre configuration, nous disposons d'un émetteur étendu (la mise au point se fait à l'œil nu), de telle sorte que nous bénéficions d'un niveau de signal confortable, malgré la densité de puissance inférieure à $1 \mathrm{~W} \mathrm{~cm}^{-2}$.

À noter que la grande étendue de l'émetteur (par rapport à la largeur de la tranche de sortie sur laquelle est réalisée la collection de lumière) ainsi que la divergence du faisceau seront la cause d'une atténuation de l'indice mesurée lorsque $L$ augmentera. Ce point, et surtout la correction à apporter pour s'en affranchir, seront détaillés dans l'annexe B. 


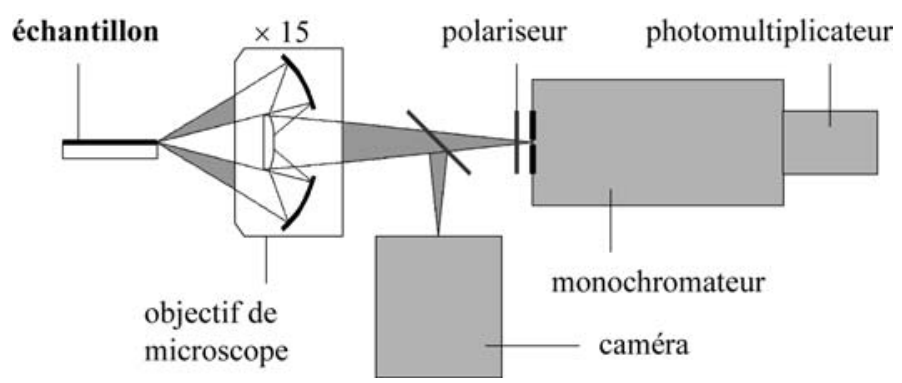

Figure 20. Montage de collection de la lumière en sortie du guide. La figure n'est pas à l'échelle.

[Setup for the collection of light outcoming from the waveguide. The figure is not to scale.]

\subsection{Mesure}

\section{Collection}

La lumière issue du guide est collectée grâce à un objectif de microscope Cassegrain de grandissement 15 (ouverture numérique 0,28), comme l'illustre la figure 20. À ce stade, on profite du fait que la lumière qui a été effectivement guidée peut être discernée spatialement de celle qui s'est propagée dans l'air ou dans le substrat. En effet, le confinement vertical qui prend fin en sortie du guide donne lieu à une importante divergence verticale du faisceau, si bien que cette lumière est vue comme étant émise par la tranche de sortie ${ }^{5}$. C'est ce faisceau que nous devons collecter.

Aussi, nous formons l'image de la tranche de sortie sur la fente d'entrée du spectromètre. Une lamelle de verre oblique, insérée sur le parcours lumineux, réfléchit environ un dixième de la lumière, afin de projeter simultanément une image identique 6 sur une caméra Hamamatsu C2400-03 à tube Vidicon, efficace à la fois dans le visible et dans le proche infrarouge $(0,4-1,8 \mu \mathrm{m})$.

Lorsque l'ensemble du montage utilise l'injection par une tranche et la collection par la tranche opposée, nous sommes dans une configuration dite end-fire, et le contrôle simultané par la caméra est indispensable car l'illumination de la tranche de sortie est le seul critère indiquant un couplage efficace à l'entrée. En outre, les positions relatives de la caméra, du spectromètre, et de la séparatrice sont réglées une fois pour toutes : on peut donc par la suite superposer virtuellement la silhouette de la fente du spectromètre sur l'image de la caméra, afin de contrôler précisément la région qui sera mesurée.

5. En comparaison, la lumière qui s'est propagée dans l'air provient du plan de la tranche d'entrée, et celle qui s'est propagée dans le substrat semble provenir d'un plan intermédiaire, en raison de la réfraction du faisceau.

6. En réalité, comme l'angle d'incidence de la lamelle est proche de l'angle de Brewster du verre, seule une des deux polarisations est réfléchie efficacement : TE lorsque l'axe de la caméra est orienté tel qu'il est dessiné sur la figure 20, TM lorsqu'il est perpendiculaire au plan de la figure. 


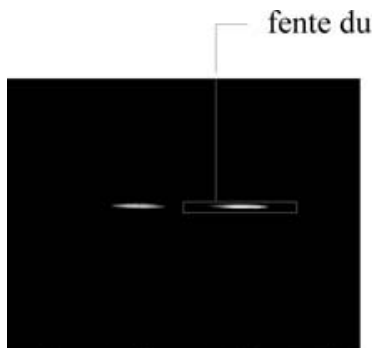

a)

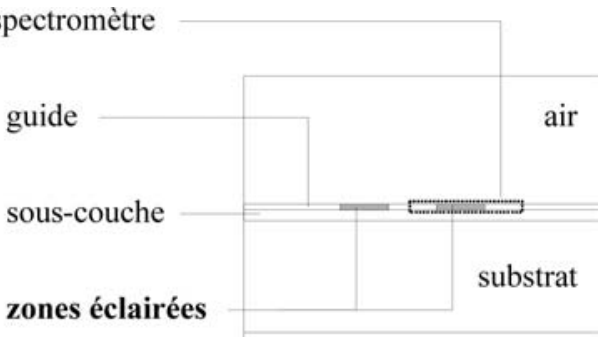

b)

Figure 21. (a) Image de la tranche de sortie du guide d'onde. Le rectangle horizontal représente la position relative de la fente du spectromètre. (b) Représentation schématique. [(a) Image of the exit edge of the waveguide. The white horizontal rectangle shows the relative position of the slit of the monochromator. (b) Schematic view.]

Une image typique enregistrée par la caméra en géométrie end-fire est reproduite sur la figure 21a. La couche guidante, horizontale, présente deux régions illuminées, en raison de la propagation oblique qui a été discutée en § 3.4. La position relative de la fente du spectromètre est indiquée par un rectangle blanc. Dans la mesure où les faisceaux guidés sont divergents, les zones illuminées peuvent être plus ou moins longues suivant la longueur du guide. Bien que réglable, la longueur de la fente nous limite cependant à l'une des deux régions.

La sélectivité spatiale est assurée à la fois en profondeur par la mise au point sur le plan de la tranche de sortie, et verticalement par la fente du spectromètre. À noter que sur l'image de la figure 21a, la restriction due à la faible épaisseur de la couche guidante peut paraître suffisante. Ce n'est pas toujours le cas : dans des conditions de couplage moins favorables, on peut observer une lumière résiduelle au-dessus du guide ou dans le substrat, et la fente permet de s'en affranchir.

En géométrie end-fire, l'efficacité du montage (abstraction faite du couplage) est directement reliée au recouvrement des ouvertures des deux objectifs (injection et collection) : dans le cas présent, nos objectifs ne sont pas identiques, mais le recouvrement des ouvertures respectives est de l'ordre de $70 \%$. Avec un émetteur interne, on ampute le signal de l'émission axiale; on peut néanmoins compter sur une efficacité de collection de quelques dizaines de pour cent.

\section{Analyse spectrale et acquisition}

L'analyse spectrale est réalisée au moyen d'un monochromateur Jobin-Yvon H20IR, de focale $200 \mathrm{~mm}$, d'ouverture $f / 4,2$, équipé d'un réseau de 600 traits par millimètre, offrant une résolution de $1 \mathrm{~nm}$, suffisante pour nos besoins. Le détecteur est un tube photomultiplicateur Hamamatsu, équipé d'une photocathode de InGaAs, et refroidi à $-80^{\circ} \mathrm{C}$ par une circulation d'azote liquide. On bénéficie ainsi simultanément d'une large plage spectrale (du visible au proche infrarouge) et d'une grande sensibilité. 
L'acquisition des spectres est pilotée par un micro-ordinateur, qui synchronise la rotation pas-à-pas du réseau, et l'acquisition du courant généré dans le photomultiplicateur, par l'intermédiaire d'un amplificateur à détection synchrone EG\&G PAR 5209 : le signal a été modulé (à environ $3 \mathrm{kHz}$ dans le cas présent) au moyen d'un hacheur placé soit devant le filament de tungstène, soit sur le parcours du faisceau de pompe, et la détection synchrone consiste à extraire du signal détecté la composante modulée à même fréquence (avec un déphasage adéquat); on parvient par ce moyen à isoler un signal noyé dans le bruit environnant (éclairage ambiant, etc.).

L'acquisition d'un spectre dure typiquement quelques minutes.

\subsection{Cas des guides multimodes}

Nous avons décrit les montages de ce chapitre en nous limitant à des considérations d'optique géométrique. Cependant, il convient de ne pas perdre de vue les propriétés de dispersion des modes guidés, particulièrement dans le cas de guides multimodes.

En effet, comme nous l'avons vu en $\S 3.2$., chapitre 2, chaque mode guidé est caractérisé par un indice effectif $n_{\text {eff }}$ qui traduit sa vitesse de phase. Lorsque plusieurs modes guidés se sont propagés sur une longueur $L$, chacun a subi un déphasage $k_{0} n_{\text {eff }} L$ qui lui est propre, si bien qu'en sortie du guide, on observe des effets analogues à ceux produits par un interféromètre de Mach-Zender à multiples voies ${ }^{7}$. Aussi, la superposition des ondes issues d'une paire de modes séparés d'un écart d'indice effectif $\Delta n_{\text {eff }}$, donne lieu à des franges d'interférences, sinusoïdales en fonction de l'inverse de la longueur d'onde et dont la période vaut

$$
\frac{1}{\lambda_{1}}-\frac{1}{\lambda_{2}}=\frac{1}{\Delta n_{\mathrm{eff}} L}
$$

Notons que nous avons supposé ici que les écarts $\Delta n_{\text {eff }}$ ne dépendaient pas de la longueur d'onde, ce qui n'est vrai qu'en première approximation. Il en est de même des indices des matériaux.

Des exemples de spectres perturbés par des franges d'interférences sont tracés sur la figure 22. Ils proviennent d'une série de mesures de transmission, réalisées sur un même guide $\mathrm{d}^{\prime}$ onde faiblement multimode, pour les deux polarisations TE et TM, et pour trois longueurs de propagation, indiquées sur chaque spectre. Étant donné la complexité du jeu de franges, nous avons eu recours à une transformation de Fourier discrète pour en extraire les différentes harmoniques. L'amplitude de la transformée de Fourier associée est tracée en face de chaque spectre. Nous avons également écrit les «fréquences » associées en termes d'écart d'indice effectif $\Delta n_{\text {eff }}$ associé, pour obtenir des tracés facilement comparables.

7. Il serait faux de parler d'interférence entre les modes guidés. En effet, les modes guidés étant orthogonaux (cf. § 3.4., Chap. 2), ils s'ignorent (comme les différentes voies d'un interféromètre); l'interférence n'a lieu qu'entre les ondes issues des différents modes guidés. 


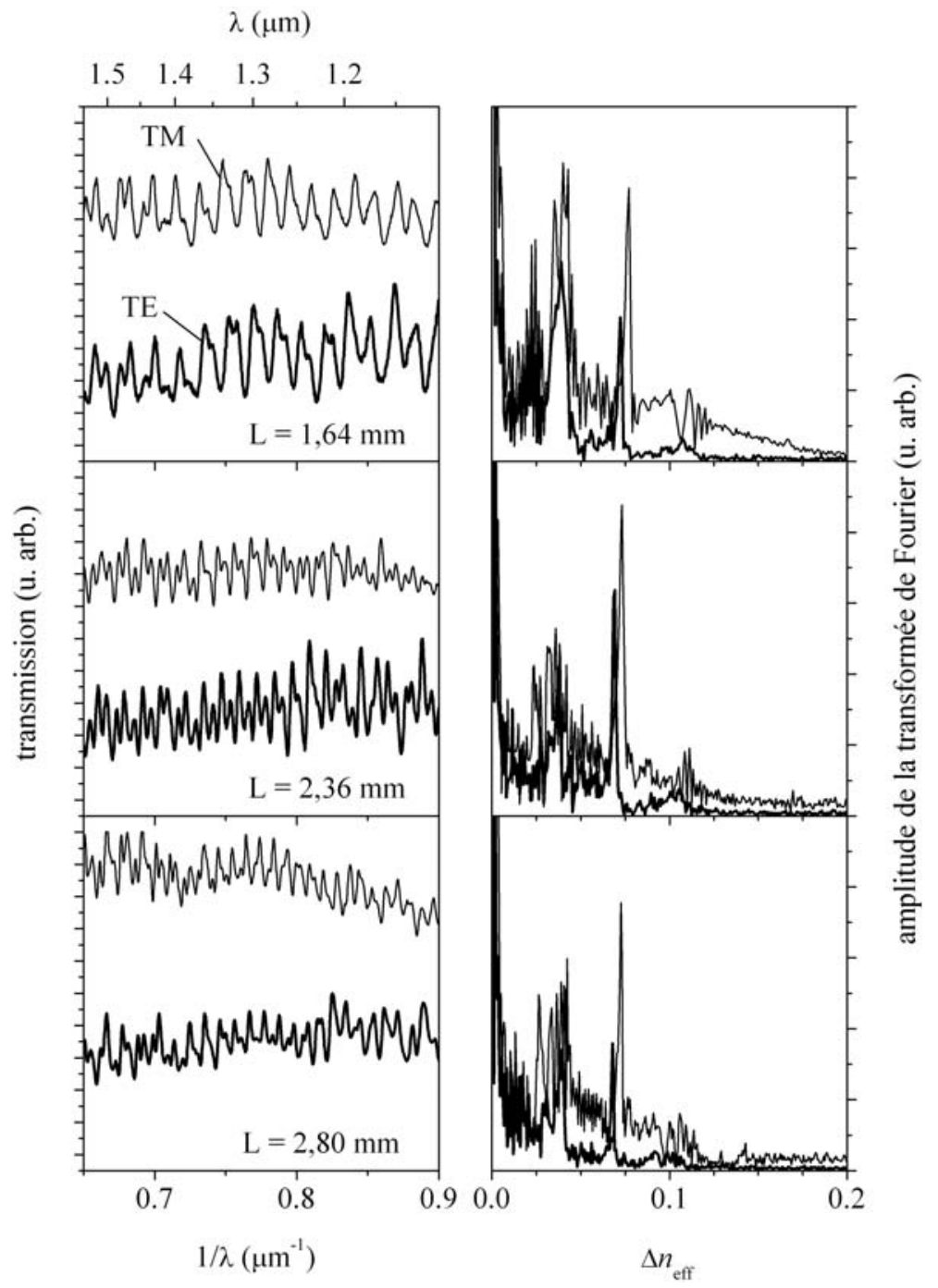

Figure 22. À gauche : spectres de transmission réalisés sur un même guide d'onde faiblement multimode, pour les deux polarisations TE et TM, et pour trois longueurs de propagation $L$ (indiquées sur chaque spectre). À droite : amplitude de la transformée de Fourier associée à chaque spectre. Les harmoniques associées sont exprimées en termes d'écart d'indice effectif $\Delta n_{\text {eff. }}$

[Left: transmittance spectra, measured on the same multimode waveguide, for both TE and TM polarisations, for three different propagation lengths $L$ (indicated close to each spectrum). Right: amplitude of the Fourier transform associated to each spectrum. The corresponding frequencies are given in terms of differences of effective index $\Delta n_{\text {eff.] }}$. 
On constate effectivement que malgré l'importante modification de l'allure des spectres lorsque la longueur de propagation augmente, la répartition des harmoniques reste parfaitement inchangée, confirmant qu'elle est associée à la structure modale du guide.

L'interprétation de ces mesures demeure néanmoins délicate, car rien ne permet d'affirmer que les différents couples de modes du guide sont représentés de façon exhaustive. À titre d'exemple, nous avons calculé que l'échantillon qui a servi aux mesures tracées sur la figure 22 possède dans cette gamme spectrale cinq modes guidés ${ }^{8}$; or, sur chaque figure, pour chaque polarisation, seuls trois pics sont clairement identifiables, et leurs valeurs permettent de les associer aux interférences dues aux trois modes d'ordres les plus bas. Notre guide possède donc cinq modes, mais seuls les trois premiers sont utilisés.

Inversement, dans nos conditions expérimentales (valeurs typiques de $\Delta n_{\mathrm{eff}}$, de $L$, et résolution spectrale), nous retiendrons qu'un spectre exempt de franges indique qu'un seul mode a transporté la lumière (sans pour autant que le guide soit monomode).

Dans ce chapitre, nous avons détaillé les méthodes expérimentales utilisées pour la formation et la structuration du silicium poreux. On retiendra leur caractère relativement simple et peu coûteux. Le facteur temps est également appréciable, puisque la durée totale de fabrication d'un échantillon ne dépasse généralement pas la demi-heure.

En dehors des techniques de caractérisation somme toute classiques comme les mesures de réflectivité ou de photoluminescence par la surface, nous avons présenté un banc expérimental satisfaisant aux contraintes propres à la géométrie guidée, mais permettant néanmoins — c'est moins courant — des mesures par la tranche de la transmission en lumière blanche ou de la photoluminescence.

8. Les paramètres du calcul sont $n_{1}=1 ; n_{2}=1,7 ; n_{3}=1,4 ; d=2,5 \mu \mathrm{m}$, avec les notations de la figure 2 . 


\section{Émission de structures planaires}

Dans ce chapitre, nous nous intéressons à l'émission du silicium poreux dans des structures planaires. Il s'agit dans un premier temps de caractériser l'émission intrinsèque du matériau d'un point de vue spectral et angulaire, afin d'évaluer la proportion relative des émetteurs orientés horizontalement et verticalement, ces derniers étant fortement impliqués dans l'émission dans le plan. Nous commençons par détailler une approche classique, proposée par Benisty et al. [69], permettant de calculer l'émission de dipôles dans un structure planaire arbitraire, puis nous la confrontons à des mesures de photoluminescence résolues en angle réalisées sur deux types de structures de silicium poreux : une couche simple, puis une microcavité.

\section{Calculs d'émission spontanée}

L'émission spontanée n'est pas une propriété immuable, mais dépend fortement de l'endroit où se trouve l'émetteur (atome, dipôle oscillant, etc.) [6]. De nombreux travaux sont menés pour étudier l'émission en milieu confiné; la motivation tient tant à une intérêt fondamental [70] que pratique, pour la réalisation de dispositifs émetteurs de lumière plus performants (lasers semiconducteurs à bas seuil [71], diodes électroluminescentes à haute brillance [72,73], sources à photon unique [74]).

\subsection{Hypothèses}

Les deux méthodes de calcul que nous présentons ici reposent sur les mêmes hypothèses, à savoir :

- la géométrie de la structure optique est supposée planaire, c'est-à-dire possédant une symétrie de translation continue le long du plan, ce qui autorise une description en termes d'ondes planes, dont la composante tangentielle du vecteur d'onde est notée $k_{\|}{ }^{1}$, les deux directions du plan étant équivalentes;

- l'émetteur est une assemblée de dipôles électriques, distribués de façon homogène dans le plan;

1. À noter que $k_{\|}$est équivalent au $\beta$ de l'optique guidée, simple question d'usage. 
- nous nous limitons à un régime de couplage faible : dans le cas d'une structure optique résonante telle qu'une microcavité, on suppose que les émetteurs qui résonnent à la même énergie que la cavité sont très minoritaires, et qu'ils possèdent en outre une durée de vie très longue. Ainsi, l'émission ne perturbe pas le mode optique de la cavité ;

- nous ne nous intéressons qu'à la puissance rayonnée en champ lointain (les effets de champ proche sont néanmoins rigoureusement pris en compte dans la structure).

\subsection{Méthode des termes sources}

La méthode des termes sources, proposée par Benisty et al. [69], repose sur un traitement classique de l'émission, au moyen de la méthode des matrices de transfert évoquée en $\S 2$., chapitre 2 (habituellement réservée au calcul de structures optiques passives), grâce à l'adjonction de termes sources.

Nous reprenons ici les étapes-clés de la méthode ${ }^{2}$. Nous donnons dans un premier temps la définition des termes sources, puis nous détaillons la résolution matricielle du champ électromagnétique. Enfin, nous donnons la relation entre champ électrique et puissance rayonnée.

\section{Définition des termes sources}

Les sources sont représentées par des discontinuités du champ électromagnétique que l'on introduit artificiellement dans le calcul, dans le plan des dipôles.

Dans un milieu infini d'indice $n_{i}$, l'émission en champ lointain d'un dipôle, selon son orientation ((v) pour un dipôle vertical, (h) pour un dipôle horizontal) et la polarisation considérée (TE ou TM), donne lieu à une puissance rayonnée par unité d'angle solide est donnée par l'une des quatre expressions suivantes :

$$
\begin{array}{ll}
\left(\frac{\mathrm{d} P}{\mathrm{~d} \Omega}\right)^{(\mathrm{v}), \mathrm{TE}}=0, \quad\left(\frac{\mathrm{d} P}{\mathrm{~d} \Omega}\right)^{(\mathrm{v}), \mathrm{TM}}=\frac{3}{8 \pi} \sin ^{2} \theta_{i}, \\
\left(\frac{\mathrm{d} P}{\mathrm{~d} \Omega}\right)^{(\mathrm{h}), \mathrm{TE}}=\frac{3}{16 \pi}, \quad\left(\frac{\mathrm{d} P}{\mathrm{~d} \Omega}\right)^{(\mathrm{h}), \mathrm{TM}}=\frac{3}{16 \pi} \cos ^{2} \theta_{i} .
\end{array}
$$

On notera que ces expressions sont normalisées, c'est-à-dire que chaque dipôle émet dans les $4 \pi$ stéradians une puissance totale unité, répartie éventuellement entre deux polarisations. En outre, les expressions données pour le dipôle (h) correspondent à une puissance rayonnée moyennée pour tous les azimuts possibles.

Les termes sources sont obtenus directement à partir des équations (5.1) comme la racine carrée de la puissance émise correspondante, pondérée par le signe adéquat déterminé par des considérations de continuité des champs. Leur expression, selon qu'elle s'applique à une onde se propageant dans le sens

2. Pour une description plus détaillée, ainsi qu'une analyse de quelques exemples, l'article original [69] est incontournable. 
$+z$ (symbolisée par $\uparrow$ ) ou $-z(\downarrow)$, est donnée par l'une des quatre relations suivantes :

$$
\begin{array}{ll}
A_{\downarrow, \uparrow}^{(\mathrm{v}) \mathrm{TE}}=0, & A_{\downarrow, \uparrow}^{(\mathrm{v}), \mathrm{TM}}=\sqrt{\frac{3}{8 \pi} \frac{k_{\|}}{k_{i}}} \\
A_{\downarrow, \uparrow}^{(\mathrm{h}) \mathrm{TE}}= \pm \sqrt{\frac{3}{16 \pi}}, & A_{\downarrow, \uparrow}^{(\mathrm{h}), \mathrm{TM}}= \pm \sqrt{\frac{3}{16 \pi}} \frac{k_{z, i}}{k_{i}} .
\end{array}
$$

L'expression des angles en termes de composantes du vecteur d'onde est un moyen commode pour traiter avec le même formalisme les ondes planes et évanescentes, étant entendu que $k_{\|}$prend toutes les valeurs permises par la couche de plus fort indice, et que c'est l'équation d'onde (Éq. (2.4)) qui décide de la valeur de $k_{z, i}$ : dans une couche non absorbante, $k_{z, i}$ vaut entre 0 et $n_{i} \omega / c$ pour une onde plane, et entre i0 et $+\mathrm{i} \infty$ pour une onde évanescente. On peut ainsi rendre compte en toute rigueur de la puissance rayonnée dans chacune des couches sans exception.

\section{Calcul du champ dans la structure}

Connaissant les termes sources, le champ électrique est calculé dans l'ensemble de la structure optique, qui est schématisée sur la figure 23, par la méthode des matrices de transfert, sachant que ce sont les valeurs à l'extérieur de la structure qui vont caractériser la puissance rayonnée.

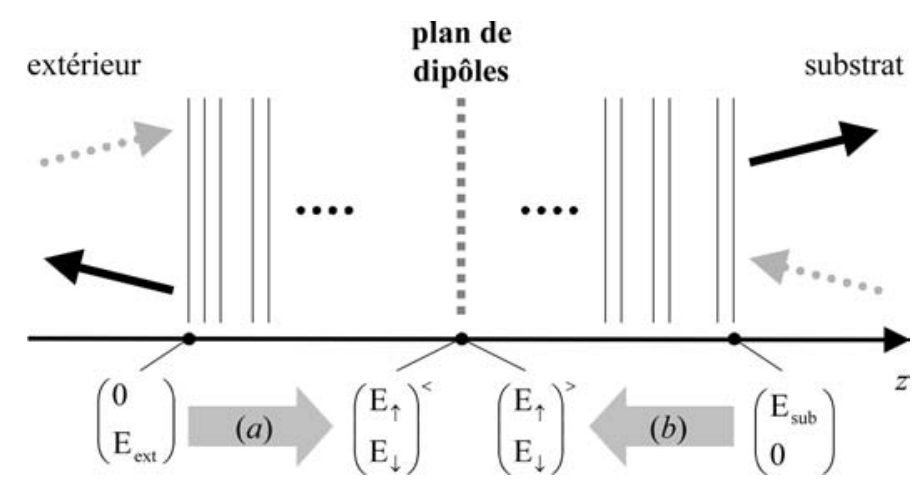

Figure 23. Représentations schématiques du plan de dipôles au milieu de la structure optique. Les ondes incidentes de part et d'autre de la structure ont une amplitude nulle (flèches en pointillés). Les amplitudes du champ en différents endroits sont également indiquées (en bas). Les termes sources introduisent une discontinuité du champ à la traversée du plan de dipôles.

[Scheme of the plane of dipoles within the optical structure. The incoming waves on both sides have an amplitude zero (dotted arrows). The amplitudes of field in several places are also indicated (bottom). The source terms give rise to a discontinuity of field at the dipole plane.] 
La localisation de l'émetteur impose en toute logique que le champ de part et d'autre de la structure soit restreint à des ondes fuyantes (schématisées par des flèches noires sur la Fig. 23). Les amplitudes associées sont notées $E_{\text {ext }}$ et $E_{\text {subr }}$, respectivement côté extérieur et côté substrat, et sont reliées au champ électrique de part et d'autre du plan de dipôles par les relations matricielles

$$
\left(\begin{array}{ll}
a_{11} & a_{12} \\
a_{21} & a_{22}
\end{array}\right)\left(\begin{array}{c}
0 \\
E_{\mathrm{ext}}
\end{array}\right)=\left(\begin{array}{c}
E_{\uparrow} \\
E_{\downarrow}
\end{array}\right)^{<}
$$

et

$$
\left(\begin{array}{ll}
b_{11} & b_{12} \\
b_{21} & b_{22}
\end{array}\right)\left(\begin{array}{c}
E_{\text {sub }} \\
0
\end{array}\right)=\left(\begin{array}{l}
E_{\uparrow} \\
E_{\downarrow}
\end{array}\right)^{>},
$$

où les éléments de matrice $a_{i, j}$ et $b_{i, j}$ sont connus et caractérisent la propagation dans chaque demi-structure, comme illustré sur la figure 23. Les termes sources, également connus, imposant la discontinuité

$$
\left(\begin{array}{c}
E_{\uparrow} \\
E_{\downarrow}
\end{array}\right)^{>}-\left(\begin{array}{c}
E_{\uparrow} \\
E_{\downarrow}
\end{array}\right)^{<}=\left(\begin{array}{c}
A_{\uparrow} \\
A_{\downarrow}
\end{array}\right),
$$

les champs à l'extérieur de la structure peuvent finalement être déterminés de façon absolue par

$$
E_{\mathrm{ext}}=\frac{b_{21} A_{\uparrow}-b_{11} A_{\downarrow}}{a_{22} b_{11}-b_{21} a_{12}},
$$

ainsi que par une expression similaire pour $E_{\text {sub }}$.

\section{Puissance rayonnée}

La normalisation des termes sources permet d'écrire la puissance rayonnée en champ lointain par unité de surface et d'angle solide dans le milieu extérieur (d'indice $n_{0}$ ) de façon particulièrement simple, à savoir

$$
\frac{\mathrm{d} P}{\mathrm{~d} \Omega \mathrm{d} s}=\left|E_{\mathrm{ext}}\right|^{2} \frac{n_{0} k_{z, 0}^{2}}{n_{i} k_{z, i}^{2}},
$$

où le second facteur prend en compte les modifications à la fois du flux du vecteur de Poynting et de l'angle solide entre le milieu de l'émetteur (indexé $i$ ) et milieu extérieur. À noter que le label «champ lointain » nécessite d'éliminer toute contribution des ondes évanescentes: celles-ci sont heureusement facilement identifiables en raison de la valeur imaginaire que prend alors $k_{z, 0}$. 


\section{Généralisation}

Cette méthode permet donc de calculer de façon rigoureuse l'émission en champ lointain d'une assemblée de dipôles électrique répartis dans une structure planaire arbitraire. Elle offre en outre une grande souplesse pour simuler les émetteurs des types les plus variés :

- une sommation adéquate de différents dipôles monochromatiques émettant à différentes longueurs d'onde permet de rendre compte de la forme spectrale voulue;

- le diagramme intrinsèque de l'émetteur est composé à partir des dipôles (h) et (v) (par exemple, l'émission d'un émetteur isotrope est obtenue en sommant la puissance émise par $\frac{2}{3}(\mathrm{~h})$ et celle émise par $\left.\frac{1}{3}(\mathrm{v})\right)$;

- une distribution en profondeur d'émetteurs incohérents est prise en compte au moyen d'une intégration du terme $\left|E_{\text {ext }}\right|^{2}$, qui se réduit généralement à une forme analytique.

On rappelle enfin qu'aucune restriction n'a été faite quant à la nature complexe des indices et vecteurs d'onde, si bien que les ondes atténuées ou évanescentes peuvent être traitées en tout généralité.

\subsection{Méthodes quantiques}

D’autres méthodes ont été développées, notamment dans le cadre des études menées sur le contrôle de l'émission spontanée dans les microcavités de semiconducteurs [71]. Elles sont généralement basées sur une description quantique de l'émission spontanée, considérant que celle-ci est stimulée par les fluctuations de point zéro du champ électromagnétique du vide.

Le calcul tel qu'il est proposé par Björk et Yamamoto fait également appel aux matrices de transfert. Les fluctuations du vide sont introduites sous la forme d'ondes incidentes de part et d'autre de la structure. Le champ résultant au niveau de l'émetteur donne directement la probabilité d'émission dans le mode considéré grâce à la règle d'or de Fermi, moyennant une opération de renversement du temps [71].

Nous avons peu utilisé cette méthode. Non pas à cause de sa nature peu intuitive, mais surtout en raison des limitations qu'elle impose dans le cas particulier des milieux à pertes, notamment quant à l'existence même de fluctuations $\mathrm{du}$ vide qui proviendraient d'un milieu semi-infini absorbant, par exemple ${ }^{3}$. En dehors de ce cas, nous avons cependant vérifié - les calculs sont strictement équivalents - qu'elle donnait des résultats strictement identiques à la méthode des termes sources.

3. Certaines publications récentes sur le sujet [75] montrent que ce problème est loin d'être trivial. 


\section{2. Émission dans une couche simple}

Une couche simple (sur son substrat de silicium) constitue la structure la plus simple que l'on puisse réaliser en silicium poreux. C'est sous cette forme que sont menées la plupart des études optiques. Nous allons cependant voir comment une étude résolue en angle permet d'apporter des informations relativement précises quant au diagramme d'émission intrinsèque du silicium poreux.

L'échantillon étudié est une couche d'une porosité de $72 \%$, d'une épaisseur de l'ordre de $3 \mu \mathrm{m}$, anodisée avec une densité de courant $j=333 \mathrm{~mA} / \mathrm{cm}^{2}$. L'indice attendu est de l'ordre de 1,3. Nous supposerons pour simplifier que l'intensité de pompage — donc la densité d'émetteurs — est homogène en profondeur ${ }^{4}$.

\subsection{Photoluminescence résolue en angle}

La série des spectres de photoluminescence obtenus au moyen du montage détaillé en $§ 2.2$., chapitre 4 est tracée pour les deux polarisations sur la figure 24 . Ils n'ont subi aucune normalisation, mais ont été corrigés par un facteur géométrique $(\cos \theta)$ de telle sorte que la surface d'échantillon analysée demeure identique quel que soit l'angle de vue ${ }^{5}$.

L'allure des spectres est classique : la bosse d'émission du silicium poreux est modulée par des franges d'interférences de type Fabry-Pérot dues à l'épaisseur finie de la couche. Lorsque l'angle augmente, les franges se décalent vers les hautes énergies, à cause de la diminution du déphasage entre les différentes ondes. En polarisation TE, les franges sont d'autant plus contrastées que l'angle augmente, en raison de l'augmentation des coefficients de réflexion, tendant vers l'unité en incidence rasante. En polarisation TM, 1'angle de $52^{\circ}$ correspondant à l'angle de Brewster de l'interface air/silicium poreux, celle-ci est traversée sans aucune réflexion, ce qui explique la disparition des franges au voisinage de cet angle. Mis à part les franges d'interférences, les séries de spectres des deux polarisations ne présentent pas de différence marquée.

\subsection{Diagramme d'émission}

Une caractérisation plus fine est obtenue en étudiant le diagramme d'émission à une longueur d'onde donnée, sur une grande plage angulaire. Le diagramme tiré des spectres de la figure 24 à $\lambda=0,7 \mu \mathrm{m}$ est tracé sur la figure 25 .

L'ajustement d'une courbe théorique sur les mesures en polarisation TE est particulièrement aisé : d'après les équations (5.2), l'émission ne fait intervenir que les dipôles (h), sans aucune dépendance d'angle ; la variation en fonction de l'angle n'est donc qu'un effet optique de la structure, dont les paramètres sont

4. En toute rigueur, l'absorption du faisceau de pompe favorise l'excitation de la partie supérieure de la couche, mais le calcul montre que la forme de l'émission résultante en est peu affectée.

5. Ceci est dû au fait que la zone émettrice projetée sur la fente du monochromateur est beaucoup plus grande que celle-ci. En fonction de l'orientation du porte-échantillon, le spectromètre voit une surface d'échantillon plus ou moins importante. 


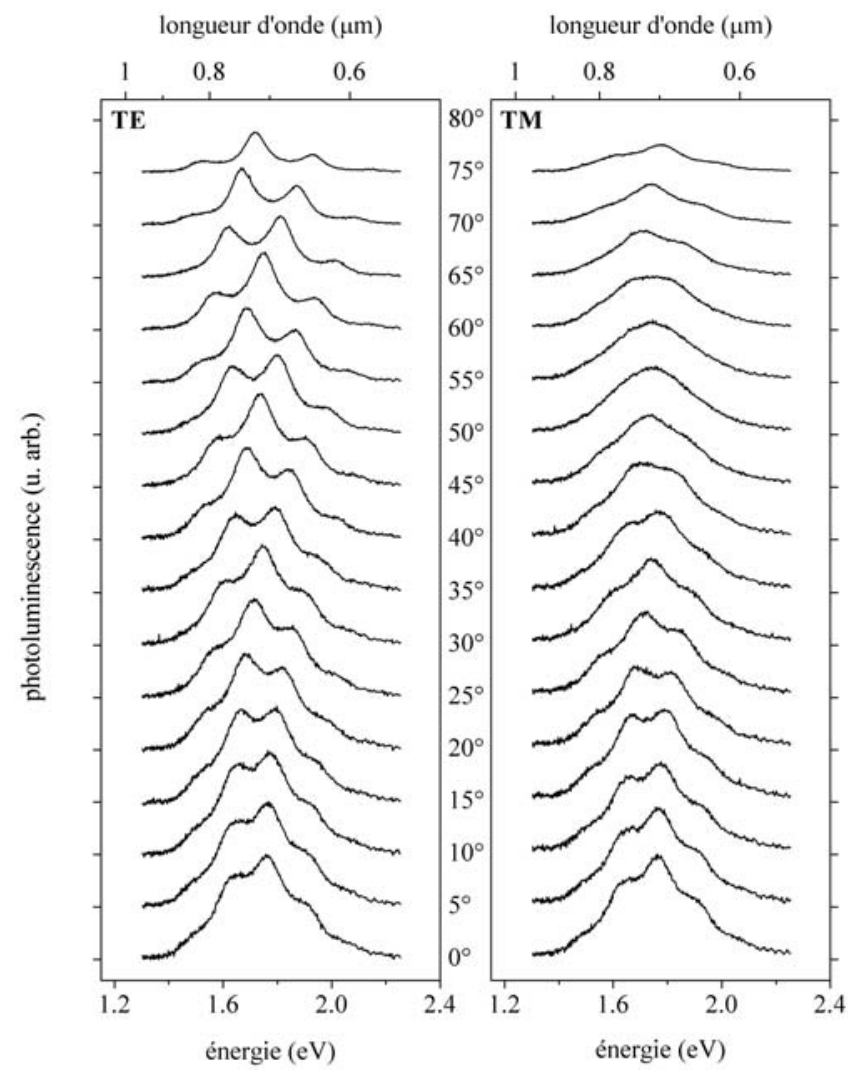

Figure 24. Série de spectres de photoluminescence obtenus à différents angles de collection pour une couche simple de silicium poreux (sur substrat), d'épaisseur $3 \mu \mathrm{m}$. Les intensités ne sont pas normalisées, mais elles ont été corrigées afin de compenser l'augmentation de surface d'échantillon analysée lorsque l'angle de collection augmente. Les spectres ont en outre été décalés verticalement pour une meilleure lisibilité.

[Set of photoluminescence spectra obtained at different collecting angles for a single porous silicon layer (on substrate) of thickness $3 \mu \mathrm{m}$. The intensities are not normalized, but have been corrected in order to compensate the increase of analyzed sample area when the collection angle increases. Spectra have been shifted vertically to be more legible.]

déterminés de façon d'autant plus précise que les franges sont marquées. Dans le cas présent, l'épaisseur de 3,11 $\mu \mathrm{m}$ et l'indice $n=1,30+0,005 \mathrm{i}$ constituent le seul couple de paramètres permettant de reproduire de façon satisfaisante les caractéristiques des franges apparaissant sur le diagramme expérimental, comme le montre la figure 25.

\subsection{Caractérisation de l'émission intrinsèque}

Le cas de la polarisation TM est légèrement plus complexe, mais surtout plus riche en informations quant à la distribution angulaire des dipôles. En effet, toujours d'après les équations (5.2), l'émission en incidence normale n'est due qu'aux 


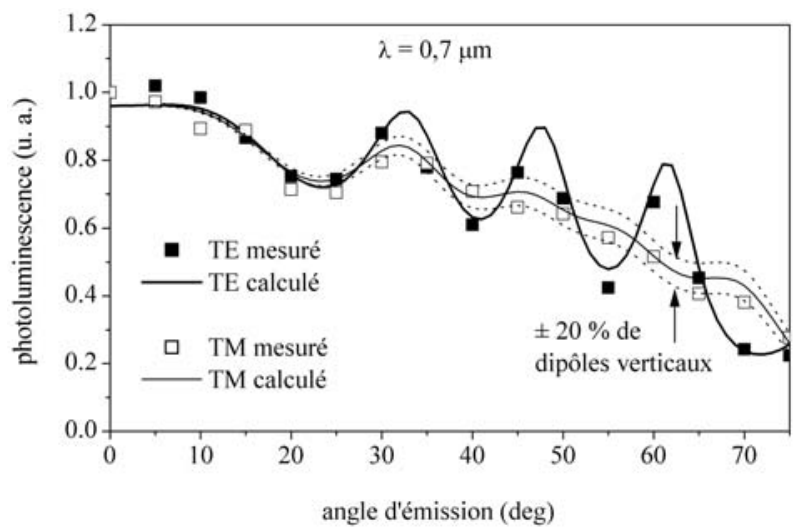

Figure 25. Diagrammes d'émission à $\lambda=0,7 \mu$ m obtenus, pour chaque polarisation, à partir de la série de spectres de la figure 24. Les carrés correspondent aux points expérimentaux, alors que le résultat du calcul est tracé en trait plein. L'émission TM résultant d'une tolérance de plus ou moins 20 \% de dipôles verticaux est indiquée par les courbes en pointillés.

[Emission pattern at $\lambda=0.7 \mu \mathrm{m}$ deduced, for each polarization, from the set of spectra of Figure 24. Squares correspond to experimental points, whereas results of calculation are plotted in solid lines. The TM intensities obtained taking into account a number of vertical dipoles of $\pm 20 \%$ are plotted in dotted lines.]

dipôles $(\mathrm{h})^{6}$; lorsque l'incidence augmente, ce sont les dipôles (v) qui prennent progressivement le relais. Les mesures en polarisation TM, sous une grande incidence sont donc une occasion unique de caractériser leur proportion. Le meilleur ajustement en polarisation TM est tracé sur la figure 25. Il a été obtenu avec une proportion $(\mathrm{v}):(\mathrm{h})=1: 2$, correspondant à une distribution isotrope des orientations. Cette valeur doit cependant être pondérée par l'incertitude de l'ajustement. Nous illustrons cette incertitude sur la figure 25 en reportant les courbes obtenues en s'accordant une tolérance de plus ou moins $20 \%$ sur le rapport (v):(h).

Des mesures plus précises ont été effectuées par Kovalev et al. [50] sur du silicium poreux de caractéristiques voisines. En excitant et en collectant l'émission par la tranche de l'échantillon, ils ont mis en évidence que l'émission par la tranche avait systématiquement une direction de polarisation privilégiée, perpendiculairement au plan de l'échantillon, dans des rapports de l'ordre de $20 \%$, quelle que soit la polarisation du faisceau de pompe. Ce phénomène est attribué à la forme ellipsoïdale des cristallites, avec une orientation préférentielle du grand axe perpendiculairement au plan [50,51].

Plus généralement, un phénomène de mémoire de polarisation a été mis en évidence : la polarisation de la photoluminescence est fortement corrélée à la

6. En tout rigueur, les dipôles (h) qui interviennent ici sont orthogonaux aux dipôles (h) de la polarisation TE. Cependant, étant donné la nature du matériau et le procédé de fabrication, ces deux directions ont de bonnes raisons d'être équivalentes. 


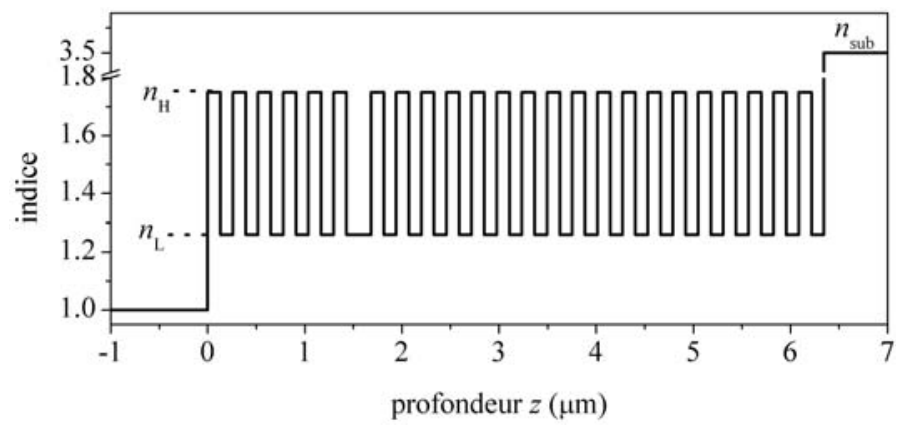

Figure 26. Profil d'indice de la microcavité. [Index profile of the microcavity.]

polarisation d'un faisceau qui a illuminé l'échantillon pendant son anodisation [76], et même, d'une façon plus dynamique, à la polarisation du faisceau de pompe [77].

Dans la mesure où ces effets restent relativement peu importants, nous nous en tiendrons à une description isotrope de l'émission du silicium poreux.

\section{Influence de la structure optique : exemple d'une microcavité}

Nous illustrons maintenant l'influence d'une structure optique sur la diagramme de rayonnement des dipôles dans le cas particulier d'une microcavité. Celle-ci est réalisée au moyen d'une séquence d'anodisations ( $c f$. 3.1., Chap. 3), à basse température pour limiter la rugosité des nombreuses interfaces [78]. La couche de porosité $70 \%\left(j=166 \mathrm{~mA} / \mathrm{cm}^{2}\right.$, indice $n_{\mathrm{c}}$, épaisseur $\left.e_{\mathrm{c}}\right)$ qui constitue la cavité demi-onde est encadrée de part et d'autre par des miroirs de Bragg, constitués d'une alternance de couches de porosité $57 \%\left(j=16,6 \mathrm{~mA} / \mathrm{cm}^{2}, n_{\mathrm{H}}, e_{\mathrm{H}}\right)$ et $70 \%(j=$ $\left.166 \mathrm{~mA} / \mathrm{cm}^{2}, n_{\mathrm{L}}, e_{\mathrm{L}}\right)$. La dissymétrie du nombre de périodes $(5,5$ au-dessus et 18 au-dessous) est prévue pour diriger préférentiellement l'émission vers l'extérieur, plutôt que dans le substrat.

\subsection{Photoluminescence résolue en angle}

La série des spectres de photoluminescence est représentée sur la figure 27. Comme précédemment, les spectres successifs ont été corrigés géométriquement et décalés verticalement, proportionnellement à l'angle de collection.

L'effet de filtrage de l'émission par la microcavité, à la fois d'un point de vue spectral et angulaire, est ici clairement mis en évidence : le mode de cavité, qui se décale vers les hautes énergies lorsque l'incidence augmente, restreint l'émission à une largeur spectrale de l'ordre de $30 \mathrm{meV}$, contre $400 \mathrm{meV}$ pour l'émission intrinsèque du silicium poreux. La bosse d'intensité qui apparaît à haute énergie, 


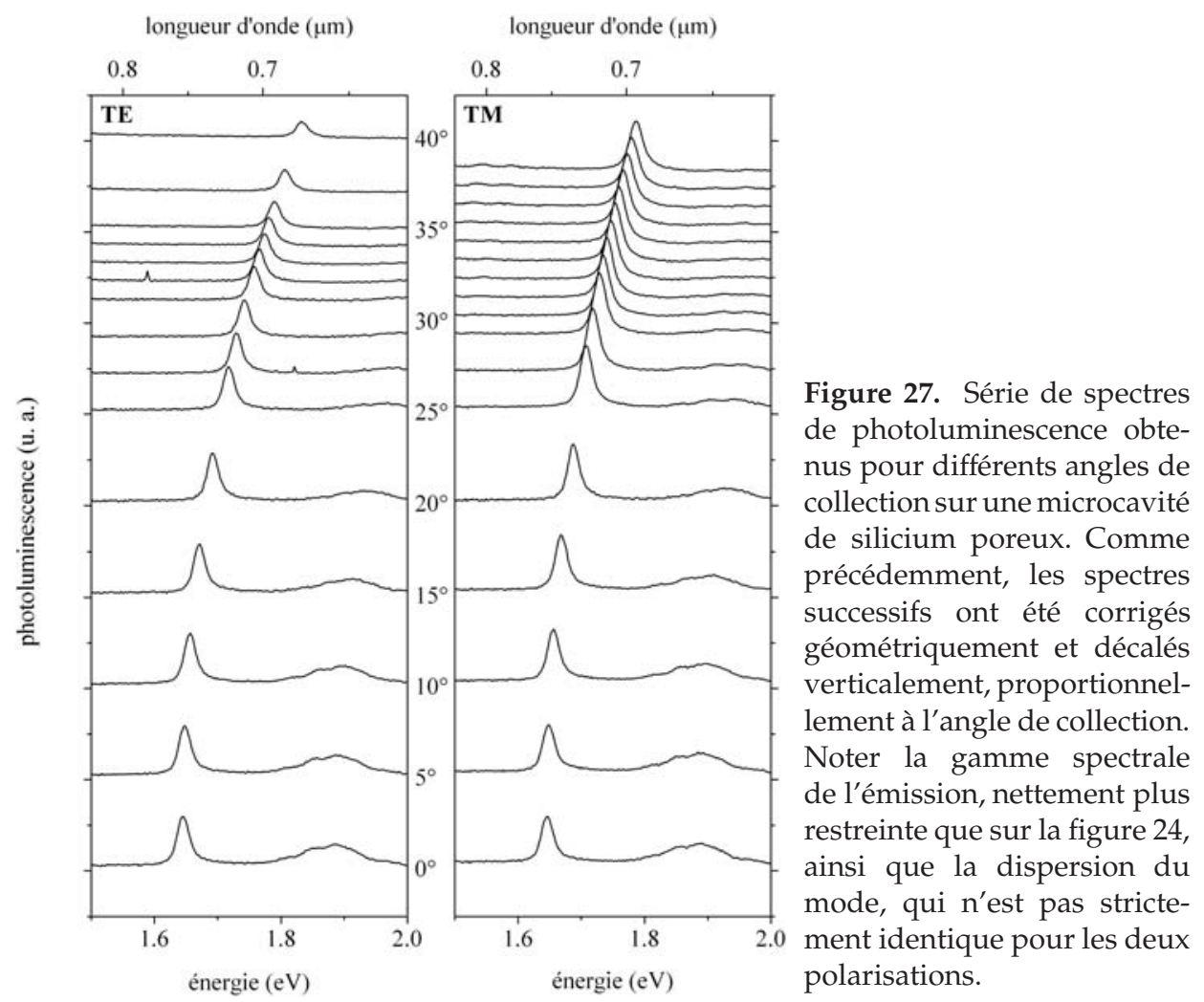

[Set of photoluminescence spectra obtained at different collecting angles for a porous silicon microcavity. Like previously, the spectra have been corrected by a geometrical factor, and shifted vertically, depending on the angle. Note the narrow spectral bandwidth, compared to Figure 24, and the mode dispersion, which is not the same for the two polarizations.]

surtout sous incidence normale, est une émission résiduelle qui n'est pas filtrée par les miroirs de Bragg, en raison de la largeur finie de la bande interdite.

\subsection{Différences liées à la polarisation}

Selon la polarisation, l'évolution angulaire des spectres diffère sur plusieurs points, que nous détaillons ici.

\section{Différences des longueurs d'onde de résonance des modes}

Bien que l'effet soit relativement discret, on peut remarquer sur la figure 27 que les modes ne suivent pas exactement la même dispersion ${ }^{7}$.

7. Le diagramme à longueur d'onde fixe (Fig. 28a) suffira à s'en convaincre. 
Dans le cas d'une microcavité demi-onde parfaite (couches des miroirs de Bragg d'épaisseurs optiques strictement équivalentes, et le double pour la cavité, ce qui n'est possible qu'à un seul angle), la longueur d'onde de résonance est simplement donnée par

$$
\lambda=4 n_{\mathrm{H}} e_{\mathrm{H}} \cos \theta_{\mathrm{H}}=4 n_{\mathrm{L}} e_{\mathrm{L}} \cos \theta_{\mathrm{L}}=2 n_{\mathrm{c}} e_{\mathrm{c}} \cos \theta_{\mathrm{c}},
$$

où $\theta_{\mathrm{H}, \mathrm{L}, \mathrm{c}}$ sont les angles de propagation dans chaque type de couche. Dès que l'angle change, elle perd toute expression analytique, et résulte d'un compromis entre les différentes valeurs d'indice et d'épaisseurs, faisant intervenir les déphasages résultant de la propagation dans chaque type de couches, ainsi que les coefficients de Fresnel à chaque interface. Étant donné que ces derniers ont une amplitude et une phase liées à la polarisation, la dépendance, à terme, de la longueur d'onde de résonance envers la polarisation n'est pas surprenante.

Par ailleurs, cet effet pourra être utilisé avec profit pour obtenir une caractérisation fine des multicouches ( $c f$. la discussion sur les limitations des multicouches, p. 23).

\section{Différences d'intensité}

L'évolution de l'intensité des spectres en fonction de l'angle diffère nettement suivant leur polarisation : diminution d'intensité en TE, semblant d'augmentation en TM. Cette observation est confirmée par le diagramme d'émission à $\lambda=0,7 \mu \mathrm{m}$, représenté sur la figure 28a : la polarisation TM bénéficie d'une émission plus intense d'environ $35 \%$.

Cependant, cet effet n'en reste pas moins cohérent avec l'émission intrinsèque relativement isotrope que nous avons caractérisée en $\S 2.3$. Pour preuve, l'ajustement ${ }^{8} \mathrm{~d}^{\prime}$ une courbe théorique, présentée en trait plein sur la même figure, montre une reproduction satisfaisante tant de la position angulaire que de la disproportion des lobes. Les émetteurs isotropes étaient supposés répartis de façon homogène sur toute l'épaisseur de la couche qui forme la cavité, ainsi que dans les couches de faible indice qui constituent les miroirs de Bragg.

En effet, dans notre structure, les couches de faible indice qui constituent à proportion de moitié les miroirs de Bragg ont été anodisées dans les mêmes conditions que la cavité. Sur l'ensemble des couches, environ une sur deux est donc susceptible d'émettre. La contribution de chaque couche à l'émission totale est tracée la figure 28b. On constate notamment que l'émission qui provient des couches supérieures (petits numéros sur la figure) est faiblement affectée par le filtrage optique de la structure. Ces couches contribuent donc fortement à l'émission à incidence faible, qui apparaît sur la figure 28a. Par contre, on constate que l'émission provenant des couches localisées au voisinage de la cavité participe à l'émission dans le mode de la cavité.

8. Les paramètres de l'ajustement sont : $n_{\mathrm{H}}=1,75+0,007 \mathrm{i} ; e_{\mathrm{H}}=0,132 \mu \mathrm{m} ; n_{\mathrm{L}}=1,26+0,007 \mathrm{i}$; $e_{\mathrm{L}}=0,127 \mu \mathrm{m} ; n_{\mathrm{c}}=n_{\mathrm{L}} ; e_{\mathrm{c}}=2 e_{\mathrm{L}} ; n_{\text {sub }}=3,5+\mathrm{i}$. 


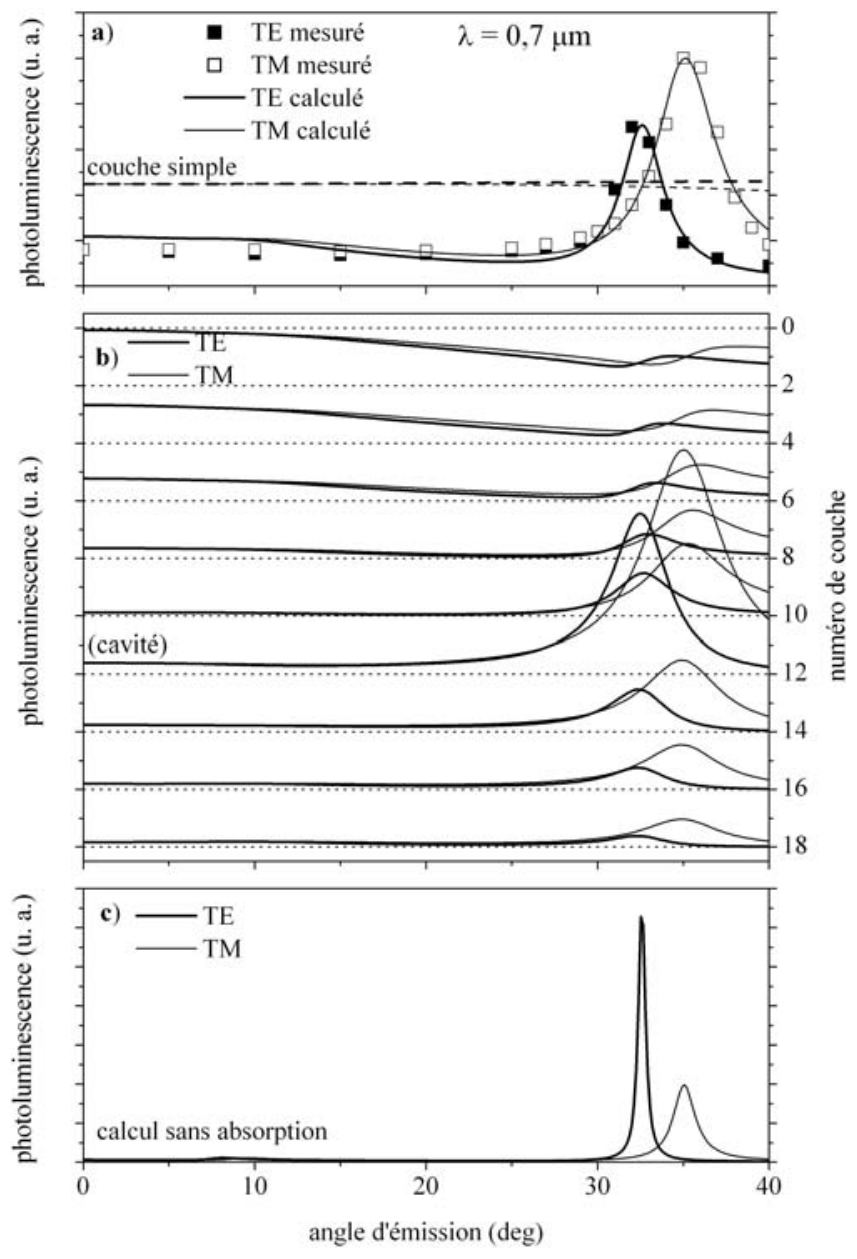

Figure 28. (a) Diagrammes d'émission à $\lambda=0,7 \mu \mathrm{m}$, obtenus pour chaque polarisation à partir de la série de spectres de la figure 27. Les carrés correspondent aux points expérimentaux, alors que l'ajustement théorique est tracé en trait plein. Le tracé en pointillés illustre l'émission d'un couche simple équivalente. (b) Contribution calculée des différentes couches (numéro indiqué à droite) à l'émission globale. Les spectres successifs ont été décalés pour une meilleure lisibilité. Étant donné leur contribution négligeable, les couches plus profondes n'ont pas été considérées. (c) Émission globale calculée en l'absence de pertes : c'est alors le mode TE qui possède la meilleure finesse.

[(a) Emission pattern at $\lambda=0.7 \mu \mathrm{m}$ deduced from the set of spectra of Figure 27. Squares correspond to experimental points, whereas the theoretical fits are plotted in solid lines. The dotted plots show the emission of a single layer of same thickness. (b) Calculated contribution of the different layers (numbers are indicated at right) to the total emission. Spectra have been shifted vertically. Due to their weak contribution, the deepest layers are not considered. (c) Total emission calculated without losses: in this case, the TE mode has the higher finesse.] 
Quant à la disproportion qui distingue les deux polarisations, elles est liée à l'absorption ${ }^{9}$, dont les effets sont décuplés à cause du grand nombre d'allers et retours imposés par la structure optique. L'absorption impose donc un seuil de réflectivité, au-delà duquel la finesse du mode reste constante, tandis que l'efficacité d'extraction se détériore rapidement. En fonction du nombre de périodes, ce seuil est atteint beaucoup plus tôt en polarisation TE, pour laquelle l'efficacité des miroirs de Bragg en incidence oblique est supérieure [4]. Dans le cas présent, le seuil a déjà été atteint en polarisation TE, ce qui justifie l'intensité plus faible. À titre de comparaison, la figure 28c illustre le diagramme d'émission calculé avec les mêmes paramètres que précédemment, mais sans absorption : le mode TE profite sans limitations de la meilleure réflectivité des miroirs.

Quoi qu'il en soit, les conséquences de l'absorption dans des structures confinées sont telles que sa prise en compte rigoureuse dans les calculs d'émission est une réelle nécessité.

\subsection{Optimisation}

En pratique, l'optimisation d'une microcavité est un compromis entre la finesse spectrale et l'efficacité d'extraction [72]. Sur la figure 28a, nous avons reporté le diagramme d'émission d'une couche simple, dont l'épaisseur est identique à la somme des couches émettrices : la redistribution spectrale induite par la microcavité apparaît nettement et le calcul montre même que notre configuration est optimale s'il s'agit de privilégier la finesse. À l'opposé, une diminution d'un facteur deux du nombre de couches du miroir de Bragg supérieur aboutit à l'efficacité d'extraction maximale (trois fois plus importante), pour une largeur certes deux fois plus importante.

Dans ce chapitre, nous avons présenté une méthode classique particulièrement intuitive et souple, permettant la modélisation en toute généralité de l'émission spontanée dans des structures planaires, même en présence d'absorption. Cette méthode nous a permis, à partir de mesures de photoluminescence résolues en angle réalisées sur une couche simple de silicium poreux, de caractériser l'émission intrinsèque du silicium poreux, dont nous retiendrons qu'elle est isotrope en première approximation. L'effet d'une structure optique sur la diagramme de rayonnement a été illustré dans le cas d'une microcavité. Nous avons mis en évidence une forte modification de la répartition à la fois spectrale et angulaire de l'émission. Nous avons également insisté sur les limitations imposées par la nature absorbante du matériau dans cette gamme spectrale.

Cependant, les effets positifs qui sont mis en évidence sur l'émission de surface ne doivent pas faire oublier qu'une bonne partie de la puissance émise reste piégée dans le plan de la structure. Cette remarque est une incitation aux études de la propagation dans le plan.

9. La valeur de 0,007 pour la partie imaginaire des indices indique une longueur effective de pénétration de l'ordre de $8 \mu \mathrm{m}$ à la longueur d'onde considérée. 


\section{Structures guidantes}

Dans la perspective du contrôle de la propagation lumineuse dans le plan, nous avons trouvé judicieux de favoriser dans un premier temps ce type de transport par un effet guidage au moyen d'une structuration verticale de l'indice. Nous dressons en premier lieu un inventaire des pertes susceptibles de limiter l'efficacité du guidage, puis nous proposons une méthode permettant de les évaluer numériquement. Enfin, deux types de structures guidantes en silicium poreux ont été élaborées et analysées.

\section{Différents types de pertes}

Les pertes optiques peuvent être attribuées à trois processus élémentaires, illustrés sur la figure 29 : absorption, diffusion et fuites (à noter que seul ce dernier type est spécifique à la géométrie guidée). Dans tous les cas, elles sont quantifiées par un coefficient $\alpha$, qui caractérise l'atténuation en $\exp (-\alpha x)$ de l'énergie transportée le long de $\hat{\mathbf{x}}$.

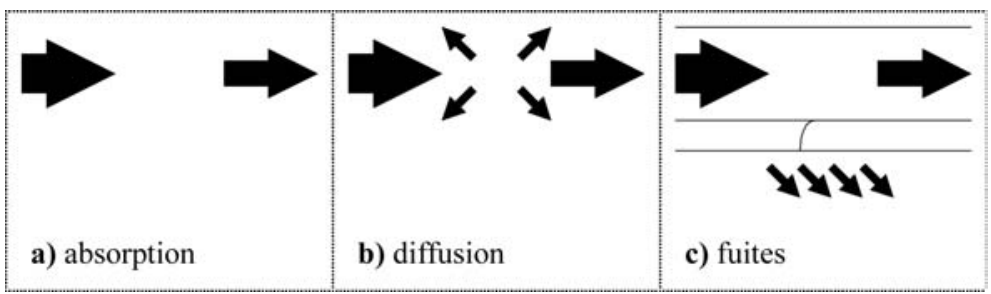

Figure 29. Différents processus qui participent aux pertes optiques dans les guides d'onde : (a) absorption, (b) diffusion, (c) fuites. Les deux premiers processus sont très généraux, le dernier est propre à la propagation guidée.

[Schemes of the different processes that contribute to optical losses in waveguides: (a) absorption, (b) scattering, (c) leakage. The first two proccesses are very general, whereas the last is related to the guiding.] 


\subsection{Absorption}

L'absorption de la lumière résulte d'un transfert de l'énergie transportée par les photons vers d'autres particules, généralement des électrons ou des phonons. Souvent négligeable dans les milieux diélectriques (on sait maintenant fabriquer des matériaux diélectriques présentant peu d'impuretés), elle joue un rôle notable dans les guides d'onde à base de semiconducteurs. Deux types d'absorption peuvent être distinguées :

- l'absorption inter-bande : l'énergie gagnée permet à des électrons de valence d'atteindre la bande de conduction. Dans les semiconducteurs à gap direct, l'énergie du gap est un seuil au-delà duquel on observe des coefficients d'absorption qui peuvent être supérieurs à $10^{4} \mathrm{~cm}^{-1}$. Dans les semiconducteurs à gap indirect (comme le silicium), le seuil est moins marqué et les valeurs plus faibles;

- l'absorption par les porteurs libres (dite aussi intra-bande) : l'énergie est transférée à des porteurs libres, ou tout au moins provenant d'états d'impuretés peu profonds. On s'en affranchit partiellement en se limitant — dans la mesure du possible - à des semiconducteurs peu dopés.

Dans le cas du silicium poreux, il est évident que les états électroniques et le gap discutés ci-dessus s'entendent pour les porteurs confinés, s'il y a lieu. En outre, on ne manquera pas de considérer l'absorption par les espèces chimiques susceptibles de tapisser la surface interne du matériau.

D'un point de vue macroscopique, l'absorption intervient par la partie imaginaire de l'indice optique $n$, l'atténuation que subit une onde plane se propageant dans un milieu absorbant massif étant caractérisée par le coefficient

$$
\alpha=\frac{4 \pi}{\lambda} \operatorname{Im}(n)
$$

La géométrie guidée permet généralement de bénéficier de longueurs de propagation nettement supérieures à celles pour un matériau massif ; signalons par exemple les études menées sur les puits quantiques de semiconducteurs [79].

\subsection{Diffusion}

La diffusion change la direction des photons, comme l'illustre la figure 29b, si bien qu'elle prélève l'énergie transportée par un mode guidé pour la redistribuer — en pure perte - dans les modes rayonnants. On distingue deux types de diffusion, selon qu'elle naît en volume ou sur une surface.

\section{Diffusion de volume}

Comme l'absorption, la diffusion de volume est attachée aux propriétés intrinsèques des matériaux utilisés. Elle est causée par les fluctuations de la constante diélectrique. Selon l'origine de ces fluctuations, la diffusion qui en résulte porte 
différents noms (Brillouin, Raman, Rayleigh), mais on montre que la dépendance spectrale de l'atténuation associée est toujours en $\lambda^{-4}$.

En optique guidée, c'est la diffusion Rayleigh qui est le plus souvent évoquée : aujourd'hui, on s'accorde à reconnaître qu'elle est responsable de la limitation des performances des meilleures fibres optiques à la valeur de $0,1 \mathrm{~dB} / \mathrm{km}$ (à la longueur d'onde de $1,55 \mu \mathrm{m}$ ) à cause des fluctuations aléatoires de densité de la silice qui les constitue [80].

Dans le cas où les fluctuations $\Delta \epsilon(\mathbf{r})$ sont faibles, à des échelles petites devant la longueur d'onde, on montre que l'atténuation associée peut s'écrire

$$
\alpha=\frac{8 \pi^{3}}{3 \lambda^{4}}\left\langle(\Delta \epsilon)^{2}\right\rangle v_{\epsilon}
$$

où le volume de corrélation $v_{\epsilon}$ quantifie l'étendue spatiale des fluctuations [81].

\section{Diffusion de surface}

Comme la diffusion de volume, la diffusion de surface est causée par des défauts aléatoires, mais ceux-ci sont localisés sur les dioptres, sous forme d'irrégularités de surface. La diffusion de surface pâtit fortement de la géométrie guidée, à cause de l'interaction continuelle de l'onde avec la surface au cours des réflexions successives.

Différents modèles ont été proposés pour quantifier les pertes en propagation causées par la rugosité d'interface. Tien [82] s'est notamment basé sur une description du faisceau guidé en termes de rayons lumineux. À partir du critère de Rayleigh, il établit qu'une onde plane de puissance $P_{0}$, dont l'incidence $\theta$ vérifie les conditions de réflexion totale, est réfléchie avec une puissance $P_{r}$, donnée par

$$
P_{r}=P_{0} \exp \left[-\left(\frac{4 \pi \sigma}{\lambda} \cos \theta\right)^{2}\right],
$$

où $\sigma$ est l'écart type des fluctuations de hauteur de la surface.

Faisant intervenir le flux de puissance guidée propre à chaque mode, Tien en tire une expression générale des pertes par unité de longueur [82]. Nous ne la donnons pas ici mais nous retiendrons que pour une rugosité donnée, les pertes sont d'autant plus réduites que

- le confinement de l'onde est faible : ceci est obtenu avec un faible contraste d'indice, le champ est étalé et «subit» peu l'interface;

- l'ordre du mode est peu élevé : l'angle $\theta$ du mode est grand, ce qui est avantageux, au vu de l'équation (6.3), d'autant que le parcours d'une même longueur nécessite alors moins de réflexions.

Le premier point cité constitue un frein certain au développement des guides à base de semiconducteurs (silicium sur silice, par exemple [83]) plus avantageux en termes d'encombrement que les guides à base de verres dopés. 


\subsection{Fuites}

Les fuites désignent le processus par lequel une quantité d'énergie peut s'échapper d'un guide sous forme de rayonnement, généralement dans le substrat, comme l'illustre la figure 29c, sans pour autant nécessiter une quelconque diffusion.

La raison de ce phénomène est la réflexion dite «totale », qui ne l'est strictement que si le milieu de faible indice qui borde la couche guidante est infini. En particulier, si un milieu d'indice supérieur ou égal à celui de la couche guidante se trouve à une distance finie, il est susceptible de prélever une certaine quantité d'énergie à la réflexion totale, qui devient alors frustrée. Ce phénomène trouve son analogie en mécanique quantique avec l'effet tunnel, qui permet à une particule de traverser une barrière de potentiel.

Dans la pratique, les fuites sont limitées en utilisant une gaine de faible indice, et dont l'épaisseur dépasse suffisamment la profondeur effective de pénétration de l'onde évanescente. Pour cette raison, si on se limite aux modes d'ordre le plus bas, loin des fréquences de coupure, la contrainte des fuites est facilement surmontable, et les fuites résiduelles sont généralement bien inférieures aux autres sources de pertes.

Remarque. D'autres pertes peuvent intervenir, si les propriétés du guide (épaisseur, indices, etc.) varient le long du parcours lumineux. Les modes qui se propagent doivent alors constamment s'adapter aux changements, au prix d'un couplages entre les différents modes guidés et rayonnants, selon des processus à rapprocher de la diffusion. D'importance secondaire, nous ne les considérons pas dans la suite.

\section{Calcul des pertes}

\subsection{Notion de mode quasi-guidé}

Nous l'avons vu, les pertes se traduisent par une atténuation de la puissance transportée dans le guide. Les modes résultant sont dits quasi-guidés et sont caractérisés par une constante de propagation complexe de la forme

$$
\tilde{\beta}=\beta+\mathrm{i} \alpha / 2,
$$

où $\beta$ est la constante de propagation réelle en l'absence de pertes ${ }^{1}$. Le caractère discret de la résonance est en outre perdu et le champ électromagnétique peut diverger à l'infini.

\section{2. Élargissement associé aux pertes}

L'élargissement du mode peut être appréhendé par une approche très générale, à rapprocher de celle qui établit la relation entre la largeur spectrale d'un mode de cavité et la durée de vie du photon confiné [16].

1. En toute rigueur, les pertes influent aussi légèrement sur la partie réelle de la constante de propagation, mais c'est un effet de second ordre. 
On considère un mode quasi-guidé, indexé $m$, se propageant dans le sens des $x$ positifs. Dans l'approximation des faibles pertes, il est très proche du mode idéal, et peut être écrit d'après l'équation (6.4)

$$
\mathbf{E}_{m}(x, z)=\mathbf{E}_{m}(z) \exp \left(\mathrm{i} \beta_{m} x\right) \exp \left(-\alpha_{m} x / 2\right),
$$

où $\mathbf{E}_{m}(z)$ est l'amplitude du champ associé au mode sans perte. Cette onde atténuée peut être décomposée sur une base d'ondes non-atténuées, chaque composante s'écrivant alors

$$
\tilde{\mathbf{E}}_{m}(\beta, z)=\frac{1}{2 \pi} \int_{0}^{+\infty} \mathbf{E}_{m}(x, z) \exp (-\mathrm{i} \beta x) \mathrm{d} x .
$$

Dans ces conditions, on peut montrer facilement que la composante longitudinale du vecteur de Poynting $\mathbf{P}$, qui quantifie le flux local d'énergie, s'écrit d'après l'équation (2.5) sous la forme

$$
P_{x}(\beta, z)=\epsilon_{0} \frac{\beta}{k_{0}}\left|\tilde{\mathbf{E}}_{m}(\beta, z)\right|^{2}=\epsilon_{0} \frac{\beta}{k_{0}} \frac{1}{4 \pi\left[\alpha_{m}^{2} / 4+\left(\beta-\beta_{m}\right)^{2}\right]}\left|\mathbf{E}_{m}(z)\right|^{2} .
$$

Pour le mode idéal, illustré figure 30a, la distribution d'énergie apparaît sous la forme d'un pic de Dirac à la valeur $\beta_{m}$. En présence de pertes, l'énergie est distribuée sur un ensemble infini de valeurs de $\beta$, comme l'illustre la figure 30b.

Cette approche permet une estimation directe des pertes : la distribution $P_{x}(\beta, z)$, qui peut être obtenue à partir des matrices de transfert, est très voisine $\mathrm{d}^{\prime}$ 'une distribution lorentzienne, dont la largeur à mi-hauteur $\Delta \beta$ donne d'après l'équation (6.7) le coefficient d'atténuation $\alpha_{m}$. Elle trouve ses limites lorsque la distribution d'énergie s'éloigne du profil lorentzien en raison de pertes trop importantes.

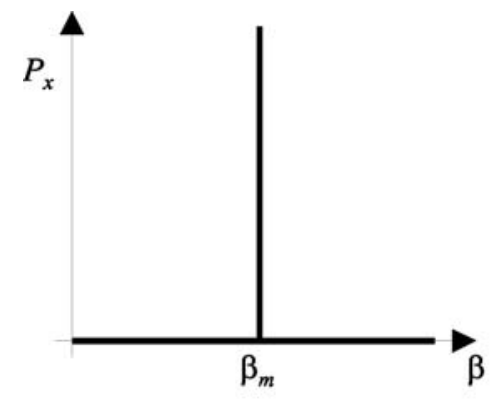

a) mode idéal

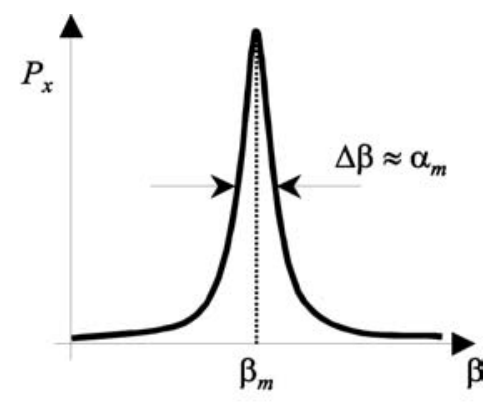

b) mode avec pertes

Figure 30. (a) Un mode idéal (sans perte) apparaît comme une singularité. (b) En présence de pertes, le mode s'élargit et la largeur à mi-hauteur du profil lorentzien est reliée au coefficient d'atténuation.

[(a) An ideal (lossless) mode appears like a singularity. (b) In case of losses, the mode acquires a Lorentzian line shape and the full width at half maximum is related to the attenuation coefficient.] 


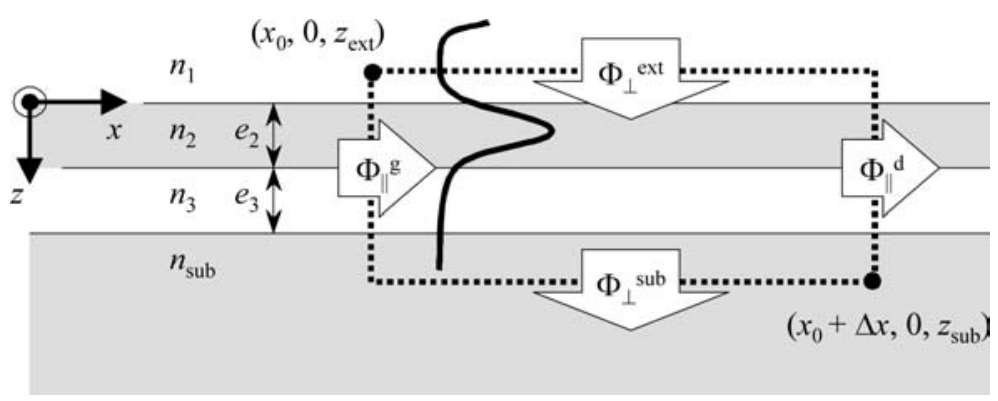

Figure 31. Vue schématique d'une structure planaire guidante. Dans le cas présent, $n_{\text {sub }} \geq$ $n_{2}>n_{3} \geq n_{1}$. Le tracé en trait épais représente la distribution du flux d'énergie (amplitude du vecteur de Poynting) dans le plan de la structure par un mode guidé. La divergence pour $z \longrightarrow-\infty$ est due au caractère fuyant du mode considéré. L'inventaire énergétique est fait sur les côtés du contour rectangulaire tracé en pointillés, et dont les coordonnées de deux points extrêmes sont indiqués. Les flèches pleines symbolisent les flux d'énergie $\Phi$ associés à chaque face.

[Schematic view of a planar guiding structure. In the present case, $n_{\text {sub }} \geq n_{2}>n_{3} \geq n_{1}$. The thick line shows the distribution of the energy flow (amplitude of Poynting vector) carried by guiding in the plane of the structure. The infinite value of field for $z \longrightarrow-\infty$ is due to the leaky nature of the mode. The balance of energy flow is taken on the sides of the dotted rectangle. Block arrows symbolize the energy flow $\Phi$ associated to each side.]

\subsection{Bilan énergétique}

Nous allons voir qu'un bilan énergétique permet d'évaluer les pertes dans un cadre plus général. Cette approche ressemble dans ses principes à celle qu'utilise Marcuse [17] pour évaluer les pertes associées aux ondes fuyantes.

Cependant, pour plus de généralité, le calcul électromagnétique est ici mené au moyen de la méthode des matrices de transfert, sur une structure planaire telle que celle qui est schématisée sur la figure 31, avec comme condition aux limites que le champ dans le substrat inférieur est limité à la seule contribution d'une onde fuyante (soit $E_{\text {sub } \downarrow}=0$, avec les notations définies en $\S 2$., Chap. 5). Tous les champs sont en outre normalisés par rapport à l'amplitude de l'onde incidente à l'interface supérieure (soit $E_{\text {ext } \uparrow}=1$ ).

Ce type de calcul, bien que tenant rigoureusement compte des pertes, est incompatible avec une description en termes d'ondes atténuées, en raison de l'hypothèse d'invariance dans le plan sur laquelle il repose. Il introduit nécessairement une compensation de la puissance transportée : l'énergie est amenée par une onde évanescente incidente, venant de l'infini ${ }^{2}$, d'où la divergence du champ au-dessus de la structure pour $z \longrightarrow-\infty$. L'analogie expérimentale de cette situation serait l'injection de lumière au moyen d'un prisme situé très loin de la surface du guide.

2. La nature évanescente de l'onde incidente n'est pas incompatible avec une flux d'énergie transversal. Cf. en annexe A l'expression du vecteur de Poynting. 
Les indices des différents milieux qui apparaissent sur la figure 31 sont tels que $n_{2}>n_{3} \geq n_{1}$, de telle sorte que le champ électrique peut être confiné dans la couche d'indice $n_{2}$. Excepté $n_{1}$, les indices peuvent être complexes. Pour envisager le cas des fuites, la gaine inférieure, $\mathrm{d}$ 'indice $n_{3}$, possède une épaisseur finie, et est suivie d'un substrat semi-infini d'indice $n_{\text {sub }} \geq n_{2}$. À noter que cette configuration a été choisie spécifiquement pour ses vertus didactiques, dans la mesure où elle correspond à un guide d'onde tout ce qu'il y a de plus conventionnel. Cependant, les expressions que nous allons établir par la suite s'appliquent à une structure planaire arbitraire, indépendamment même de son aptitude à guider la lumière. La seule restriction est la nature réelle de l'indice $n_{1}$ du milieu extérieur.

\section{Différents flux énergétiques}

Le bilan énergétique est réalisé sur le contour rectangulaire tracé en pointillés sur la figure 31. Le positionnement vertical ainsi que la hauteur du rectangle sont choisis pour pouvoir comptabiliser l'ensemble de l'énergie transportée dans le plan : si le substrat est parcouru par une onde évanescente, la profondeur $z_{\text {sub }}$ est choisie pour être la plus importante possible; si le substrat est parcouru par une onde propagative, $z_{\text {ext }}$ peut être choisi juste au-dessous de la dernière interface : dans le milieu extérieur, la limite $z_{\mathrm{ext}}$ est choisie pour contenir au maximum l'onde évanescente issue du guide, en limitant l'influence de la divergence ${ }^{3}$. Quant à l'étendue horizontale $\Delta x$ du contour, elle est supposée infinitésimale.

Étant donné l'invariance latérale de notre système (que ne détruit pas l'injection continue de lumière par le haut), il est évident que le flux entrant à gauche est égal en valeur absolue au flux sortant par la droite. En prenant comme convention de donner une valeur positive à tout flux entrant, on a

$$
\Phi_{\|}(\beta)=\Phi_{\|}^{\mathrm{g}}(\beta)=-\Phi_{\|}^{\mathrm{d}}(\beta)=\int_{z_{\text {ext }}}^{z_{\text {sub }}} P_{x}(\beta, z) \mathrm{d} z
$$

où $P_{x}$ est la composante dans le plan du vecteur de Poynting $\mathbf{P}$, dont l'expression dans le formalisme des matrices de transfert est établie dans l'annexe A. Dans ces conditions, les flux perpendiculaires s'écrivent simplement

$$
\Phi_{\perp}^{\mathrm{ext}}(\beta)=P_{z}\left(\beta, z_{\mathrm{ext}}\right) \Delta x=P_{z}^{\mathrm{ext}}(\beta) \Delta x
$$

et

$$
\Phi_{\perp}^{\mathrm{sub}}\left(\beta, z_{\mathrm{sub}}\right)=-P_{z}\left(\beta, z_{\mathrm{sub}}\right) \Delta x .
$$

La notation $P_{z}^{\text {ext }}$ indique que $P_{z}$, et donc $\Phi_{\perp}^{\text {ext }}$ ne dépendent pas du choix de $z_{\text {ext }}$, puisque le milieu extérieur n'est pas absorbant.

3. Dans le calcul numérique, cet arbitraire n'intervient pas, car l'expression analytique du vecteur de Poynting permet le calcul du flux guidé par une intégration rigoureuse, en se limitant à la composante qui décroît en $-\infty$. 


\section{Signification des différents flux}

Le flux $\Phi_{\|}$représente l'énergie qui est transportée dans le plan, et dont la valeur est conservée tout au long de la propagation.

Si l'onde dans le substrat est évanescente, le flux $\Phi_{\perp}^{\text {sub }}$ est nul, car aucune énergie n'est transportée perpendiculairement au plan ( $c f$. Annexe A). Donc $\Phi_{\perp}^{\text {sub }}$ ne comptabilise que le rayonnement dans le substrat, donc les fuites (quitte ensuite à ce que ce rayonnement soit absorbé par le substrat).

Reste le flux $\Phi_{\perp}^{\text {ext }}$. En l'absence de perte dans le guide, la conservation de l'énergie lui impose une valeur nulle. En cas de pertes, seul ce flux permet de les compenser. Il est donc caractéristique des pertes en général, que l'énergie ait été consommée dans le guide (absorption, diffusion), ou qu'elle se soit échappée dans le substrat.

\section{Calcul du coefficient d'atténuation}

Connaissant l'énergie transportée $\Phi_{\|}^{\mathrm{g}}$, nous avons donc accès à la part $\Phi_{\perp}^{\text {ext }}$ perdue lors d'une propagation sur une distance $\Delta x$. L'ensemble des pertes qui contribuent à l'atténuation sont donc quantifiées par le coefficient

$$
\alpha_{m}=\frac{1}{\Delta x} \frac{\Phi_{\perp}^{\mathrm{ext}}\left(\beta_{m}\right)}{\Phi_{\|}\left(\beta_{m}\right)}=\frac{P_{z}^{\mathrm{ext}}\left(\beta_{m}\right)}{\Phi_{\|}\left(\beta_{m}\right)}
$$

dans lequel la contribution des fuites est donnée par

$$
\alpha_{m}^{\text {fuites }}=\lim _{z_{\text {sub }} \longrightarrow+\infty} \frac{1}{\Delta x} \frac{\Phi_{\perp}^{\mathrm{sub}}\left(\beta_{m}, z_{\mathrm{sub}}\right)}{\Phi_{\|}\left(\beta_{m}\right)}=\lim _{z_{\mathrm{sub}} \longrightarrow+\infty} \frac{P_{z}\left(\beta_{m}, z_{\mathrm{sub}}\right)}{\Phi_{\|}\left(\beta_{m}\right)} .
$$

L'équation (6.11) donne donc accès à la partie imaginaire de la constante de propagation du mode quasi-guidé, selon l'équation (6.4).

\subsection{Exemples}

Nous illustrons cette méthode dans le cas de deux configurations : le guide à fuites et le guide absorbant.

\section{Guide à fuites}

La structure considérée est un guide planaire multimode, dont l'épaisseur de la gaine inférieure est finie, ce qui donne lieu à des fuites vers le substrat de fort indice. Avec les notations de la figure 31, les paramètres du calcul sont $n_{1}=1$, $n_{2}=1,8, n_{3}=1,5$ et $n_{\text {sub }}=1,8$ pour les indices, et $e_{2}=3 \mu \mathrm{m}$ et $e_{3}=1 \mu \mathrm{m}$ pour les épaisseurs. La longueur d'onde est $\lambda=1,3 \mu \mathrm{m}$, et nous nous limitons par souci de simplicité à la polarisation TE.

Les flux énergétiques, pris sur les contours dessinés sur la figure 31, ont été calculés sur toute la gamme de $\beta$ permis par la couche guidante, et leurs variations sont reportées sur la figure 32. Les lignes verticales en pointillés, correspondant 

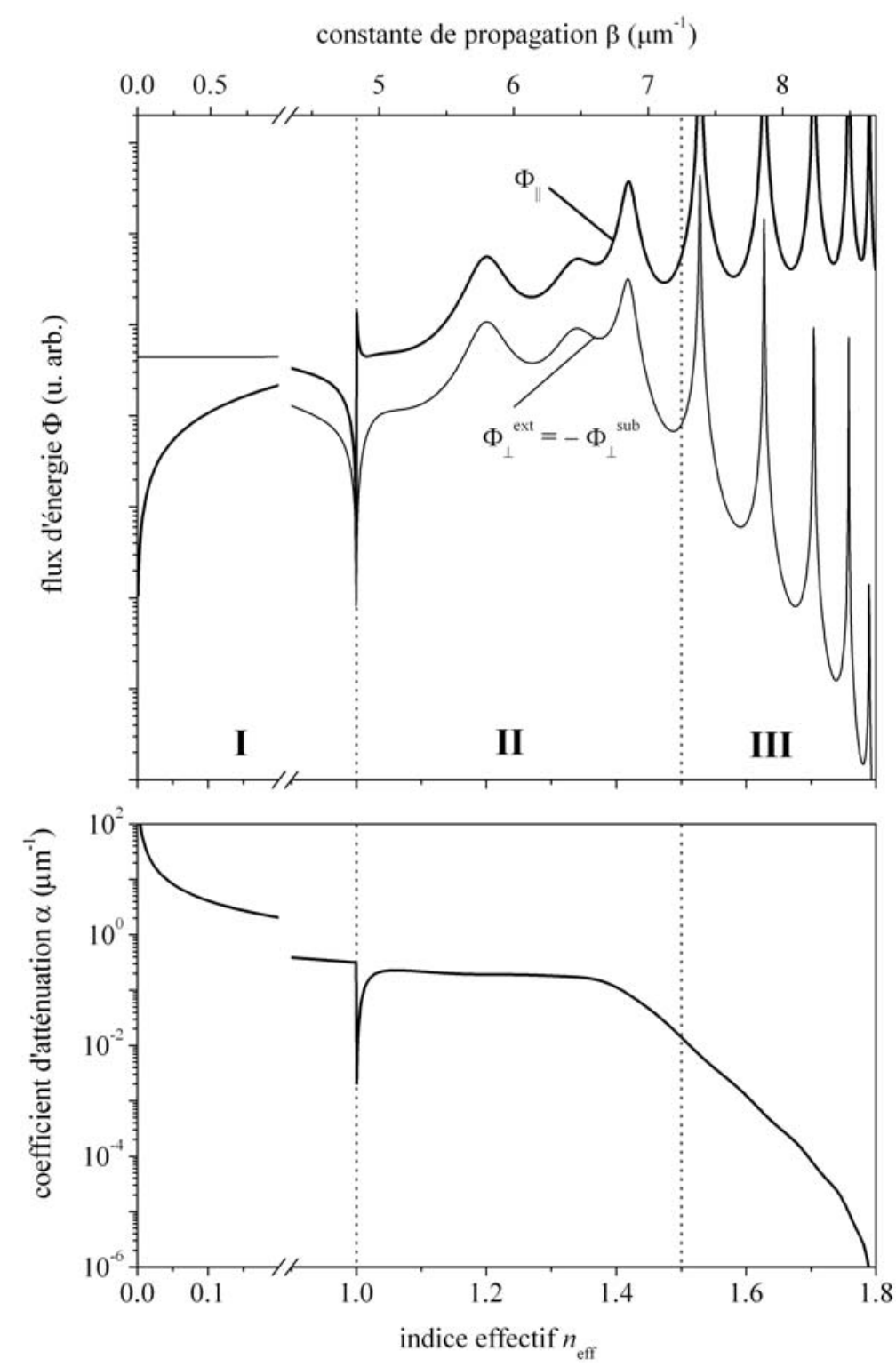

Figure 32. Propagation dans un guide à fuites (la gaine a une épaisseur finie, et le fort indice du substrat admet des ondes rayonnantes). En haut : évolution des différents flux énergétiques en fonction de la constante de propagation. En bas : coefficient d'atténuation associé. Polarisation TE uniquement.

[Propagation in a leaky waveguide (the cladding has a finite thickness and power is radiated in the high index substrate). Top: plot of the different energy flows vs. the propagating constant. Bottom: associated attenuation coefficient. TE polarization only.] 
à $n_{\text {eff }}=n_{1}$ et $n_{\text {eff }}=n_{3}$, permettent de scinder le diagramme en trois régions :

- à gauche, la région $\mathrm{I}\left(n_{\mathrm{eff}}<1\right)$ correspond au continuum de modes rayonnant dans l'ensemble de la structure;

- au centre, la région II $\left(1<n_{\text {eff }}<1,5\right)$ correspond aux modes dits de substrat, la condition de réflexion totale n'étant vérifiée qu'à l'interface supérieure de la couche guidante ;

- à droite, la région III $\left(1,5<n_{\mathrm{eff}}<1,8\right)$ contient cinq pics, conséquences des cinq modes quasi guidés.

À noter que sur le diagramme, les tracés passent de la région II à la région III sans aucune modification notable, ce qui souligne la transition progressive entre les deux régimes. En outre, le guide ne dissipant pas d'énergie, l'apport de l'extérieur $\left(\Phi_{\perp}^{\text {ext }}\right)$ compense exactement les fuites dans le substrat $\left(\Phi_{\perp}^{\text {sub }}\right)$.

Le coefficient d'atténuation déduit de l'équation (6.11) est tracé sur la courbe du bas. Malgré la grande dynamique des deux flux à comparer, le rapport est caractérisé par une évolution nettement plus monotone : 1'atténuation ne dépend pas de façon critique de la valeur de la constante de propagation. Sur une grande plage, l'effet le plus notable est la diminution drastique des pertes lorsque l'ordre des modes diminue (soit lorsque $n_{\text {eff }}$ augmente). Comme nous l'avons annoncé en $\S 1.3$., les modes d'ordre peu élevés sont favorisés car les ondes évanescentes de part et d'autre de la couche guidante sont étalées dans une moindre mesure, ce qui diminue leur tendance à fuir.

\section{Guide absorbant}

Le guide présenté ici ne diffère du précédent que par deux points : la gaine inférieure a une épaisseur $\left(e_{3}\right)$ infinie, jouant le rôle de substrat à faible indice, et les seules pertes sont dues à l'absorption dans la couche guidante, dont l'indice vaut ici $n_{2}=1,8+0,001$.

Les flux d'énergie calculés sont reportés sur la figure 33. Ils ne présentent pas de différence notable par rapport au cas précédent, si ce n'est qu'aucune énergie ne traverse la structure, puisqu'elle est consommée dans la couche guidante.

Des différences plus notables apparaissent sur le coefficient d'absorption : dans la région III, où le confinement vertical est le plus efficace, les pertes ont une valeur quasiment constante, très voisine de la valeur que donne l'équation (6.1) pour une onde plane dans un matériau massif de même indice que la couche guidante.

Des arguments simples permettent de voir cette faible variation comme le résultat de deux effets qui se compensent. En effet, les modes d'ordre peu élevé sont avantagés en termes de pertes dans la mesure où le rayon lumineux associé progresse dans la couche guidante sous une incidence très rasante, ce qui limite le chemin total parcouru dans le matériau absorbant. Inversement, les modes d'ordre plus élevés sont caractérisés par un étalement vertical plus important dans les couches environnantes (non-absorbantes), et bénéficient ainsi d'une part plus importante de puissance non-atténuée. Dans le cas présent, les deux effets 

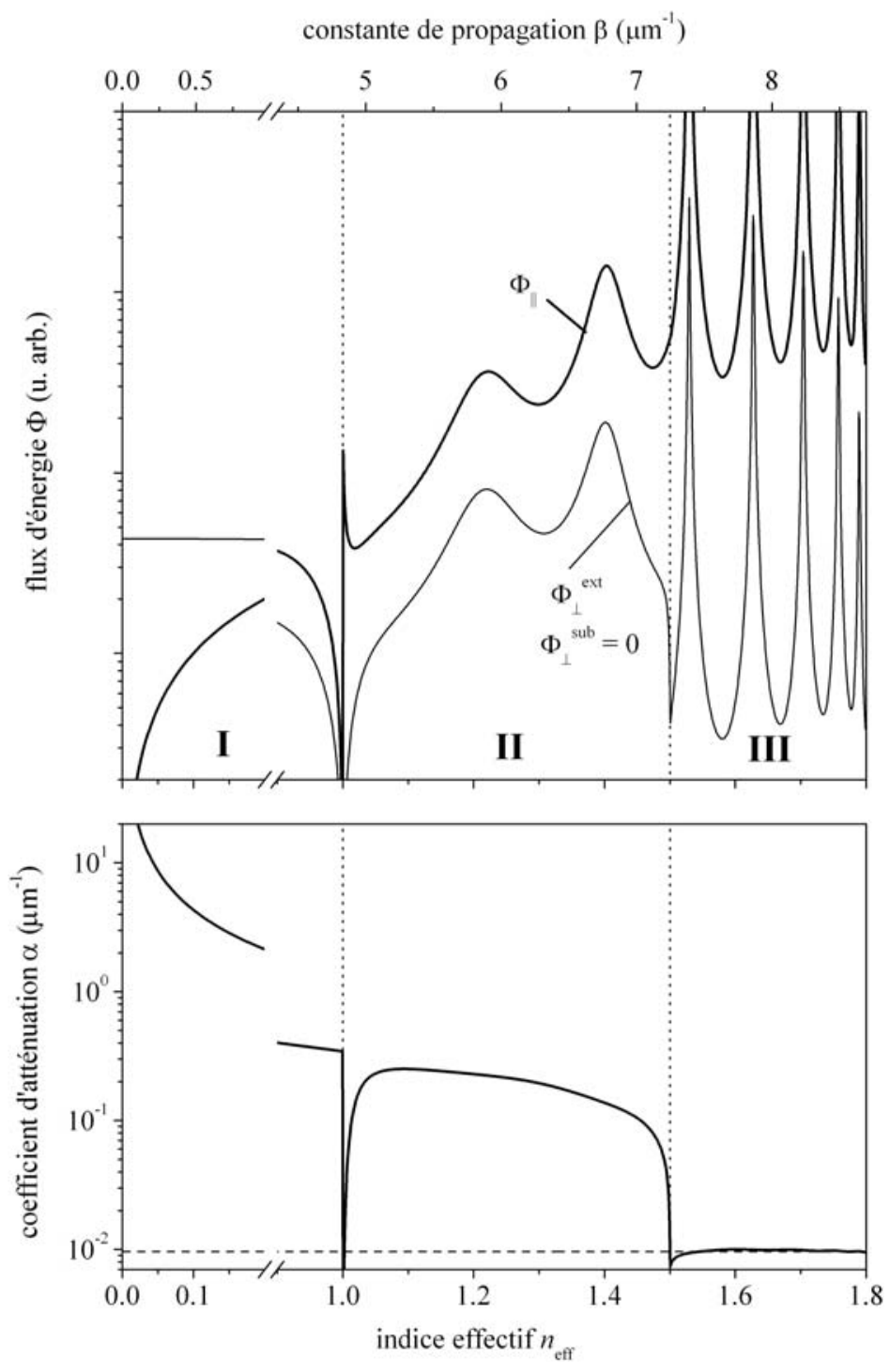

Figure 33. Propagation dans un guide absorbant. En haut : évolution des différents flux énergétiques en fonction de la constante de propagation. En bas : coefficient d'atténuation associé. La ligne horizontale en tirets représente le coefficient d'atténuation dans un matériau massif de même indice que la couche guidante. Polarisation TE uniquement.

[Propagation in an absorbing waveguide. Top: plot of the different energy flows vs. the propagating constant. Bottom: associated attenuation coefficient. The horizontal dashed line represents the attenuation coefficient in a bulk material of same index as the guiding layer. TE polarization only.] 
se compensent quasiment, et l'atténuation effective, tantôt supérieure (pour $n_{\mathrm{eff}} \simeq$ 1,6 ), tantôt inférieure (pour $n_{\mathrm{eff}} \simeq 1,8$ ) ne se démarque que très légèrement de sa valeur dans le matériau massif.

Ces deux exemples illustrent le caractère universel de cette méthode, qui permet de traiter dans le même cadre formel différentes sources de pertes, sans sacrifier à la généralité des structures optiques, grâce au formalisme des matrices de transfert.

\section{3. Étude d'un guide à saut d'indice}

Nous présentons maintenant une étude expérimentale des pertes menée sur un guide planaire en silicium poreux. La structure guidante proposée n'apporte aucune innovation d'un point de vue optique, mais la motivation est autre : d'un point de vue purement expérimental, il s'agit de valider le montage d'optique guidée en lumière blanche proposé en $\S 3$., chapitre 4 ; d'un point de vue plus fondamental, on attend des réponses quantitatives quant aux limites du matériau dans une utilisation en optique guidée.

\subsection{Description de l'échantillon}

\section{Choix des porosités}

Avec la perspective d'une structuration de l'indice dans le plan, il nous a paru judicieux de réaliser le guidage dans un couche possédant un indice le plus élevé possible, soit une porosité faible. Nous avons écarté d'emblée les couches anodisées à trop faible densité de courant, en raison de la grande rugosité d'interface qui les handicape. Avec une densité de courant supérieure, les conditions choisies ( $j=16,6 \mathrm{~mA} / \mathrm{cm}^{2}$, porosité de $58 \%$ ) permettent d'atteindre un indice $n_{2}=1,77$, dans de bonnes conditions de reproductibilité.

En raison de l'indice élevé du substrat $\left(n_{\text {sub }} \simeq 3,5\right)$, une couche (gaine) est requise sous la couche guidante. Le confinement demande un indice le plus faible possible, mais nous avons été contraint de nous limiter à des couches d'indice $n_{3}=1,67\left(j=50 \mathrm{~mA} / \mathrm{cm}^{2}\right.$, porosité de $\left.65 \%\right)$. En effet, des couches d'indice plus faible (donc de porosité plus importante) se sont révélées inutilisables aux épaisseurs souhaitées, pour des raisons de tenue mécanique (rupture de la couche inférieure et décollement de la couche supérieure).

\section{Choix des épaisseurs}

Étant donné le couple d'indices disponibles, le nombre de modes guidés qui peuvent exister dans la structure est directement lié au rapport $e_{2} / \lambda$, où $e_{2}$ est l'épaisseur de la couche guidante. La dispersion du guide, calculée en supposant la gaine infinie et en résolvant l'équation transcendante des modes [84], est tracée en fonction de ce rapport sur la figure 34. 


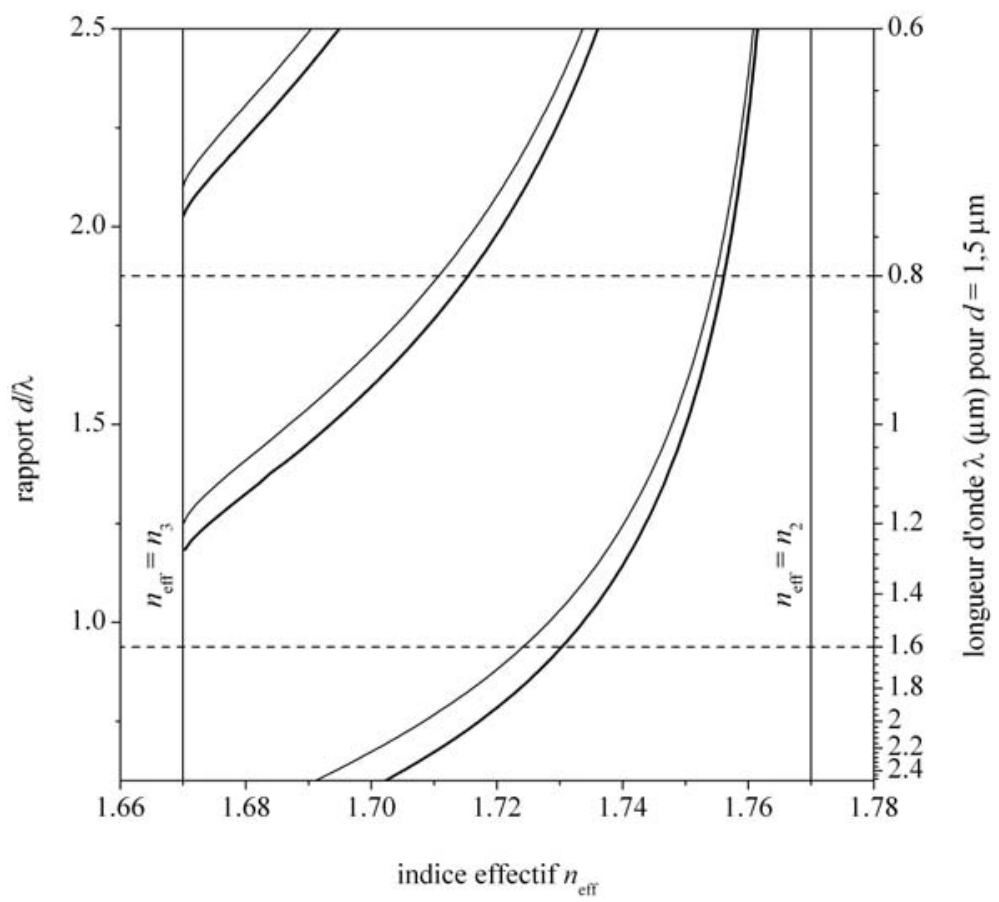

Figure 34. Courbes de dispersion d'un guide planaire dont les indices sont $n_{2}=1,77$ et $n_{3}=1,67$. L'échelle verticale de gauche est en fonction du rapport $e_{2} / \lambda$. L'échelle de droite donne la longueur d'onde sachant que $e_{2}=1,5 \mu \mathrm{m}$. Les deux lignes horizontales en tirets représentent les limites de notre gamme spectrale d'étude. Les deux points noirs indiquent les deux modes TE à $\lambda=1 \mu \mathrm{m}$, dont la distribution de champ électrique sera tracée sur la figure 35 .

[Dispersion curves of a planar waveguide with the following indices: $n_{2}=1.77$ and $n_{3}=1.67$. The unit of the left vertical axis is the ratio $e_{2} / \lambda$. The right axis gives the wavelengh, knowing that $e_{2}=1.5 \mu \mathrm{m}$. The two horizontal dashed lines show the limit of our spectral range. The two black points indicate the two TE modes at $\lambda=1 \mu \mathrm{m}$ from which the field distribution is plotted in Figure 35.]

Il apparaît notamment qu'un guidage monomode nécessite que $e_{2} / \lambda<1,2$, soit une épaisseur $e_{2}$ inférieure au micron si nous voulons tendre vers les longueurs d'onde du visible. Nous avons cependant constaté qu'une telle épaisseur rendait le couplage par la tranche délicat et peu efficace. Aussi, nous avons préféré tolérer l'existence d'un deuxième mode, en choisissant une épaisseur $e_{2}=1,5 \mu \mathrm{m}$, plus proche de la taille de l'image du filament que nous projetons sur la tranche d'entrée.

Dans ces conditions, dans la fenêtre spectrale d'étude limitée par les lignes horizontales pointillées sur la figure 34, on peut distinguer une région monomode $(1,2 \mu \mathrm{m}<\lambda<1,6 \mu \mathrm{m})$ et une région bimode $(0,8 \mu \mathrm{m}<\lambda<1,2 \mu \mathrm{m})$. Dans cette dernière situation, les deux modes TE à $\lambda=1 \mu \mathrm{m}$, signalés par deux points noirs, 

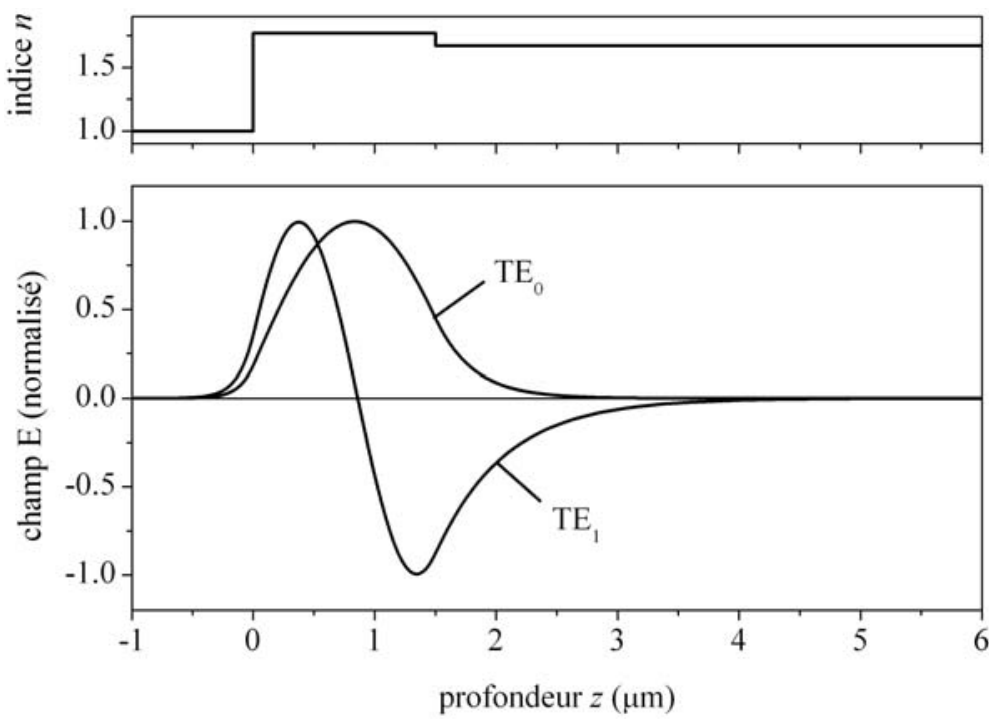

Figure 35. Distributions des champs électriques associés aux deux modes TE présents dans la structure guidante proposée à $\lambda=1 \mu \mathrm{m}$. Les deux courbes sont normalisées. Les deux modes TM, non-représentés, possèdent une distribution du champ magnétique de forme tout à fait voisine.

[Distribution of the electrical field associated to the two TE modes that exist in the proposed structure at $\lambda=1 \mu \mathrm{m}$. Both curves are normalized. The two TM modes are not plotted, the distribution of magnetic field is however very similar.]

correspondent à une distribution de champ électrique en profondeur tracée sur la figure 35. Dans la mesure où notre injection de lumière est symétrique par rapport au plan de la couche, il est raisonnable de penser que la majorité de la lumière va être couplée dans les modes fondamentaux $\left(\mathrm{TE}_{0}\right.$ ou $\left.\mathrm{TM}_{0}\right)$, et qu'ainsi nous pourrons bénéficier d'une propagation limitée à un seul mode.

Quand à l'épaisseur $e_{3}$ de la gaine inférieure, son choix a été davantage guidé par des arguments géométriques (limiter au maximum l'injection directe de la lumière dans le substrat, lors du couplage), que par un souci de limiter les fuites. Sinon la valeur retenue de $4,5 \mu \mathrm{m}$, à laquelle le calcul attribue des pertes dans le pire des cas $(\lambda=1,6 \mu \mathrm{m}, \mathrm{TM})$ de l'ordre de $10^{-3} \mathrm{~cm}^{-1}$, pourrait sembler à juste titre quelque peu démesurée!

\subsection{Mesure de transmission}

Des mesures de transmission on été réalisées en lumière blanche sur deux guides d'ondes, de longueurs $L_{1}$ et $L_{2}$, provenant du même échantillon de silicium poreux. Pour garantir des conditions de couplage équivalentes, ce sont les interfaces issues de la même ligne de clivage qui ont été utilisées dans chaque cas pour l'injection. En outre, le réglage de l'injection de lumière et l'acquisition ont été 
systématiquement reproduits plusieurs fois, après déréglage. Ainsi, si un spectre d'intensité anormalement faible se distingue du lot, la mesure correspondante est invalidée. Les mesures retenues, qui correspondent au couplage optimal, présentent généralement un écart relatif d'intensité de l'ordre du pour cent, et sont moyennées.

Les spectres de transmission obtenus sont tracés pour chaque polarisation sur la figure 36. On rappelle qu'ils ont été corrigés par la réponse de l'ensemble du montage, mesurée sans échantillon.

Aucune modulation d'intensité n'est constatée, ce qui indique une propagation monomode dans les faits. Si une part notable de l'énergie guidée avait été transportée par le second mode, l'écart d'indice effectif qui apparaît sur la figure 35 indique, d'après l'équation (4.2), que les oscillations de l'intensité auraient eu dans le cas présent une période de l'ordre de la dizaine de nanomètres, quantité tout à fait mesurable.

\subsection{Mesure des pertes}

Pour les deux polarisations, l'allure des spectres est comparable, et la diminution de l'intensité $I$ induite par la différence de longueur est suffisamment notable pour permettre une détermination précise du coefficient d'atténuation, d'après

$$
\alpha=-\frac{\ln \left[I\left(L_{2}\right)\right]-\ln \left[I\left(L_{1}\right)\right]}{L_{2}-L_{1}} .
$$

Le résultat de cette opération appliquée aux spectres de transmission est reporté sur la figure 37. Les valeurs des deux polarisations sont confondues. Nous avons également reporté sur le même graphe les valeurs du coefficient d'absorption, mesurées sur du silicium poreux par effet mirage par Vincent et al. [86], et sur du silicium massif par photoluminescence par Daub et al. [85]. À noter que chaque fois, le dopage du silicium est comparable à celui que nous utilisons.

Les deux courbes d'absorption «pure » sont typiques du silicium poreux : le seuil d'absorption du matériau poreux apparaît décalé vers les hautes énergies, conformément aux prévisions du modèle du confinement quantique ; par contre les valeurs à faible énergie indiquent une forte absorption résiduelle, par rapport au matériau massif. L'interprétation de cette absorption demeure controversée, bien que des mesures en fonction de la température incitent à les décrire comme une conséquence du confinement quantique [87].

L'ensemble des pertes mesurées dans le guide d'onde en silicium poreux suivent un comportement similaire, à ceci près qu'elles sont supérieures de plus d'un ordre de grandeur. 


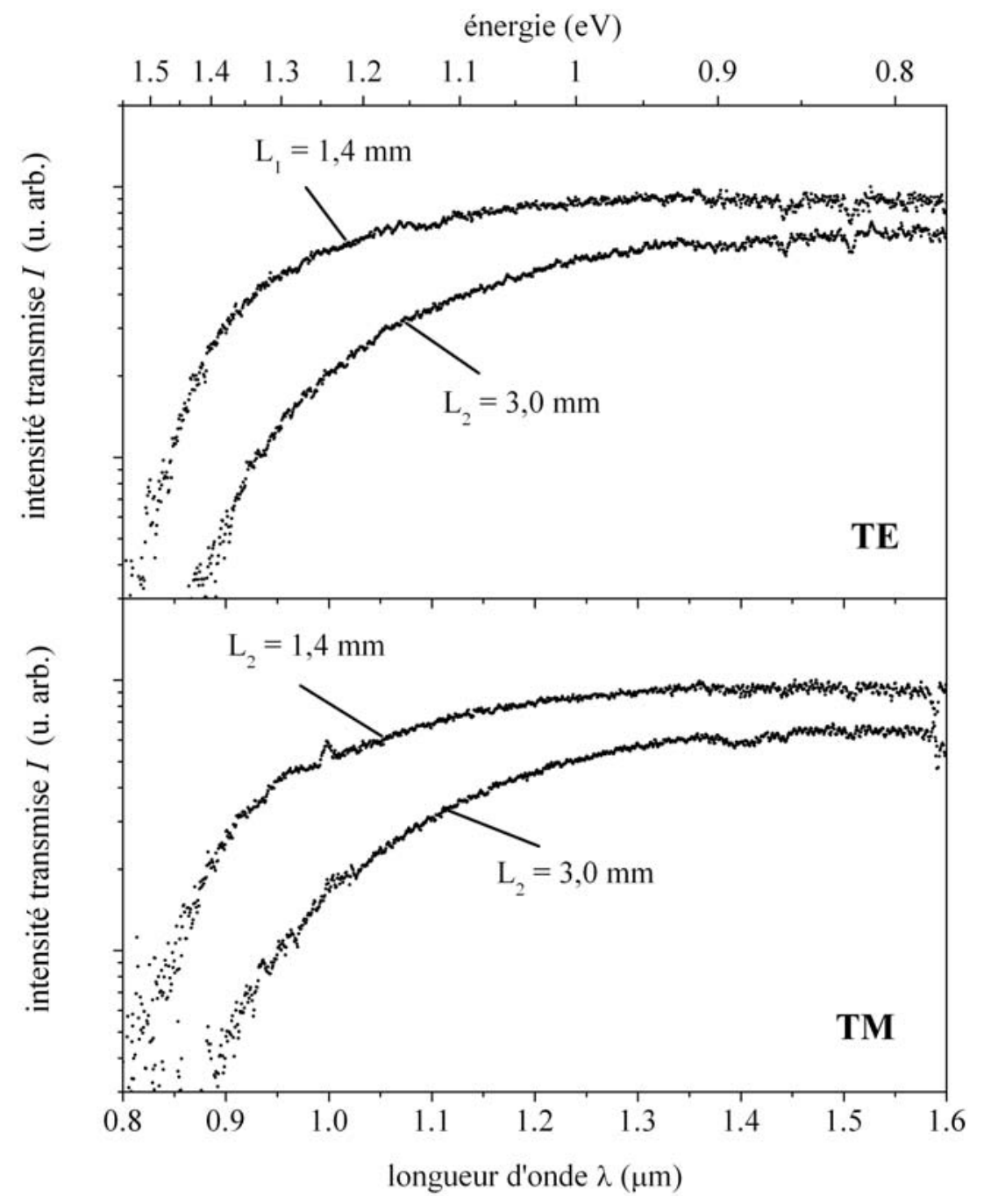

Figure 36. Spectres de transmission mesurés pour les deux polarisations sur deux guides d'ondes différant par leur longueur, indiquée sur chaque tracé. Les faibles oscillations régulières (dont la période ne dépend pas de la longueur de guide), que l'on distingue en polarisation TM pour 1,4 $\mu \mathrm{m}<\lambda<1,6 \mu \mathrm{m}$, sont dues aux interférences produites par les réflexions sur les deux faces parallèles de la séparatrice utilisée pour la visualisation à la caméra (cf. à ce sujet la note 6, page 38 ).

[Transmittance spectra measured for both polarizations on two identical waveguides of different length, indicated on each plot. The weak oscillations (those period does not depend on the waveguide lenght) that can be seen in TM polarization for $1.4 \mu \mathrm{m}<\lambda<$ $1.6 \mu \mathrm{m}$ are due to interferences produced by the reflections on the two parallel sides of the beamsplitter used for the camera monitoring (see also note 6, page 38).] 


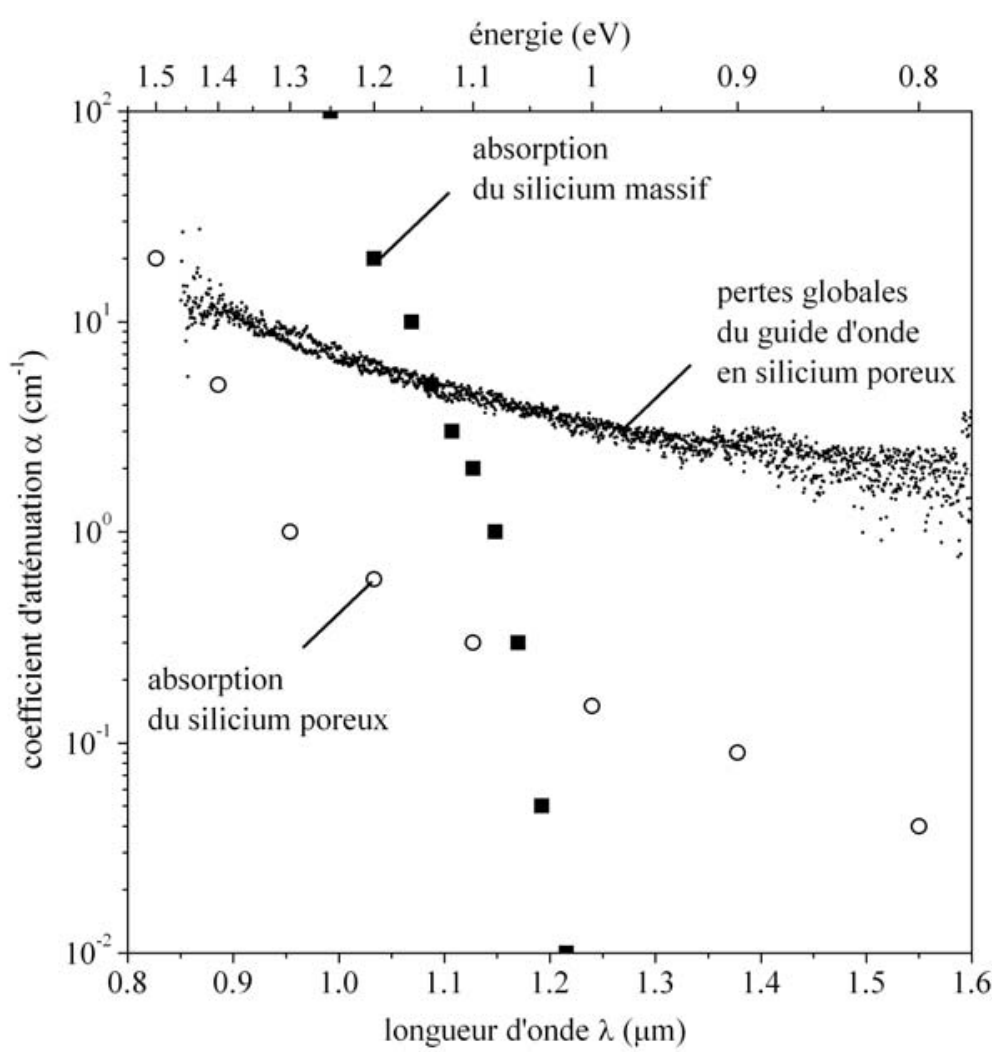

Figure 37. Pertes déduites des spectres de transmission (tracé en points noirs). Les valeurs obtenues pour les deux polarisations sont confondues. Les coefficients d'absorption du silicium massif [85], ainsi que ceux du silicium poreux, mesurés par effet mirage [86], sont également reportés.

[Losses deduced from the transmittance spectra. The values for the two polarizations are superposed. Absorption coefficients of bulk silicon [85] and porous silicon (measured by photothermal deflection spectroscopy [86]) are also plotted.]

\section{4. Évaluation des différentes sources de pertes}

\section{Absorption}

Les valeurs de l'absorption que nous avons sont relativement faibles, et nous avons vu en $\S 2.4$. que la propagation guidée ne générait pas d'augmentation notable de l'atténuation ${ }^{4}$. Dans ces conditions, elle n'intervient dans l'ensemble des pertes que dans des proportions minimes.

4. À $\lambda \sim 1,3 \mu \mathrm{m}$, le coefficient d'absorption du matériau $\alpha \sim 10^{-1} \mathrm{~cm}^{-1}$ correspond à $\operatorname{Im}\left(n_{2}\right) \sim 10^{-6}$, et le calcul de l'atténuation en géométrie guidée donne $\alpha \sim 10^{-1} \mathrm{~cm}^{-1}$. 


\section{Diffusion de surface}

La rugosité de l'interface inférieure des couches de silicium poreux est une propriété connue, et ses effets sur la diffusion de la lumière ont fait l'objet de nombreuses études [66]. Bien que les valeurs typiques soient tabulées, nous avons préféré la mesurer dans notre configuration spécifique.

Pour ce faire, nous avons anodisé une couche simple de silicium poreux dans des conditions électrochimiques strictement identiques à celles utilisées pour l'anodisation de la couche supérieure de la structure guidante, afin d'obtenir la même porosité, et surtout la même épaisseur. La couche obtenue est ensuite dissoute dans un bain de soude $(0,1 \mathrm{M})$, découvrant ainsi à l'air libre l'interface incriminée, qui peut alors être étudiée au moyen d'un profilomètre Tencor P-10. Une mesure réalisée en plusieurs endroits de la surface permet de mesurer une rugosité moyenne, dont l'écart type vaut $\sigma=7,5 \mathrm{~nm}$.

Cette valeur peut alors être introduite dans les équations de continuité du calcul [67], la perturbation à ajouter étant déduite de l'équation (6.3). Les pertes calculées sur les modes $\mathrm{TE}_{0}$ et $\mathrm{TM}_{0}$ sont tracées sur la figure 38. Les valeurs

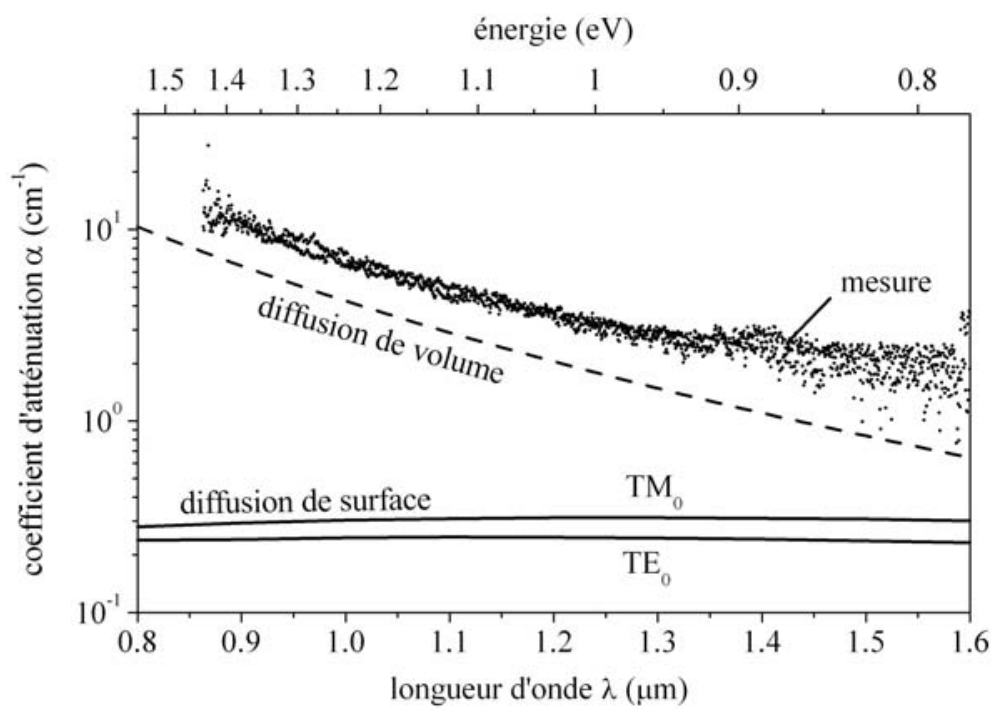

Figure 38. Confrontation des pertes mesurées avec des estimations numériques pour différentes sources possibles : diffusion de surface, et diffusion de volume. Les pertes dues à l'absorption ainsi que celles dues aux fuites, nettement inférieures, ne sont pas reportées. [Comparison between measured losses and numerical estimations for several possible sources: surface scattering and volume scattering. Absorption losses and leakage, are not plotted because very low.] 
calculées sont assez faibles $\left(\alpha^{\mathrm{TE}} \simeq 0,2 \mathrm{~cm}^{-1}, \alpha^{\mathrm{TM}} \simeq 0,3 \mathrm{~cm}^{-1}\right)^{5}$, et ne dépendent quasiment pas de la longueur d'onde. À noter que dans l'équation (6.3), si la longueur d'onde apparaît de façon explicite, elle intervient aussi dans l'angle $\mathrm{d}$ 'incidence du mode, qui augmente de façon monotone lorsque $\lambda$ diminue. On constate en définitive que ces deux effets se compensent.

\section{Diffusion de volume}

La diffusion de volume est rarement évoquée dans le cas du silicium poreux, celuici étant généralement considéré en optique comme parfaitement homogène, dans la mesure où les inhomogénéités sont de taille nanométrique.

À si petite échelle, les propriétés diélectriques sont très complexes à modéliser [88]. Néanmoins, si l'on s'en tient à un assemblage de cristallites de même indice que le silicium $(n \simeq 3,5)$, sachant que leur diamètre moyen est d'environ $4 \mathrm{~nm}$ [89], on peut calculer grossièrement les pertes associées. Bien que le domaine de validité de l'équation (6.2) soit largement dépassé, nous avons tracé le résultat du calcul sur la figure 38 .

Dans de telles conditions d'approximation, nous n'avons pas cherché à ajuster la courbe théorique sur les données expérimentales. Le résultat n'en reste pas moins convaincant, tant dans les ordres de grandeurs que dans la dépendance spectrale.

La diffusion Rayleigh n'a pour ainsi dire jamais été discutée dans le cadre des études optiques menées sur le silicium poreux. Il est vrai que la configuration présentée ici est originale : la géométrie guidée permet de sonder de grandes distances, dans la gamme de transparence du matériau; en outre, les structures guidantes déjà réalisées faisaient généralement appel à du silicium poreux totalement oxydé et densifié [90], aux propriétés finalement très proches de la silice, avec une constante diélectrique sans doute plus homogène à l'échelle du nanomètre.

\subsection{Mise en évidence de la diffusion}

Pour mettre en évidence la diffusion, nous avons analysé la lumière qui peut être collectée par la tranche, hors du faisceaux guidé, dans le même plan. Nous avions en effet observé une diffusion de la lumière lors de l'injection au moyen d'un faisceau laser $(\lambda=1,3 \mu \mathrm{m})$ : pour un couplage optimal, toute la tranche du guide, ainsi que la surface (probablement inférieure?) de la couche guidante s'illuminaient, sans pour autant pouvoir quantifier de façon fiable la lumière diffusée.

En lumière blanche, la faible intensité (environ deux ordres de grandeur plus faible à proximité immédiate du faisceau direct) ne permet pas une visualisation à la caméra, mais est néanmoins détectable par le photomultiplicateur. Le signal obtenu est tracé sur la figure 39.

5. À titre de comparaison, les valeurs calculées pour le mode suivant sont typiquement dix fois plus importantes. 


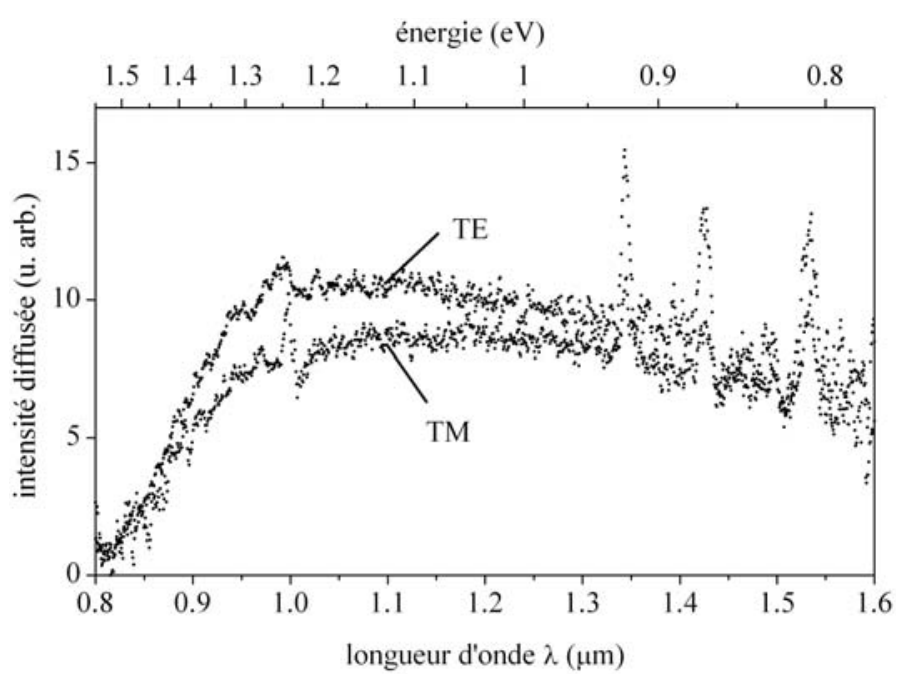

Figure 39. Intensité détectée par la tranche à proximité du faisceau guidé, dans le même plan.

[Intensity outcoming from the output edge, close to the guided beam, in the same plane.]

Mis à part les trois pics dans la gamme 1,3-1,6 $\mu \mathrm{m}$, dont la présence exclusive et systématique sur les mesures de diffusion nous conduit à suggérer un phénomène de diffusion résonante (par des espèces chimiques adsorbées dans le matériau?), la dépendance spectrale permet de distinguer ces spectres de ceux mesurés sur le faisceau direct, notamment en raison de la diminution d'intensité pour $\lambda>1,2 \mu \mathrm{m}$.

Ce comportement est cohérent avec une interprétation en termes de diffusion : à petite longueur d'onde, la lumière n'est pas diffusée, car absorbée ; progressivement, la diffusion prend le dessus, et on la détecte; enfin, à grande longueur d'onde, son efficacité diminue.

À ce stade, le rôle joué par la diffusion ne peut pas à ce stade être remis en cause ; les estimations attribuent un rôle prépondérant à la diffusion de volume, même si une dépendance spectrale en $\lambda^{-4}$ constituerait une preuve supplémentaire. En outre, si les mesures quantitatives de lumière diffusée sont délicates à mener en optique en général, la géométrie guidée apporte son lot de complexité supplémentaire.

\section{4. Étude d'un guide à bande interdite photonique}

\subsection{Principe}

Dans les structures guidantes que nous avons considérées jusqu'à présent, le confinement était dû à des réflexions totales internes, la lumière étant alors confinée dans le milieu de plus fort indice. 
On peut cependant envisager de remplacer ce type de réflexion par une réflexion de Bragg, sur un empilement diélectrique périodique. L'idée, proposée dans les années 70 par Yeh et al. [91], n'a pas véritablement suscité d'enthousiasme de part de la communauté de l'optique guidée de l'époque. Plus récemment, dans la mouvance des cristaux photoniques, les progrès théoriques et techniques accomplis (miroirs diélectriques omnidirectionnels [92,93], fibres optiques photoniques [94]) ont vu d'un bon œil la propagation alors possible dans un milieu de faible indice, synonyme de pertes réduites.

Dans ce contexte, grâce à son aptitude à la réalisation de structures multicouches, le silicium poreux paraît parfaitement adapté. En outre, la nature émettrice, propre aux couches de faible indice (forte porosité), constitue un avantage certain pour les caractérisations.

\subsection{Description de l'échantillon}

La structure que nous proposons peut être apparentée à une microcavité privée de son miroir supérieur, le guidage se faisant dans la couche de la cavité, par réflexion totale contre l'interface supérieure au contact de l'air, et par réflexion de Bragg sur le miroir multicouche inférieur, qui s'oppose ainsi à la pénétration de lumière.

La résonance (donc le mode «guidé ») nécessite au minimum un cavité de type demi-onde, pour laquelle la longueur $\mathrm{d}^{\prime}$ onde $\lambda_{0}$ de résonance vérifie ${ }^{6}$

$$
n_{\mathrm{c}} e_{\mathrm{c}} \cos \theta_{\mathrm{c}}=\lambda_{0} / 2=2 n_{\mathrm{H}} e_{\mathrm{H}} \cos \theta_{\mathrm{H}}=2 n_{\mathrm{L}} e_{\mathrm{L}} \cos \theta_{\mathrm{L}}
$$

où les indices $\mathrm{H}$, L et c se rapportent respectivement aux couches de fort indice, faible indice, et à la couche de la cavité, les angles associés étant au-delà de la valeur critique de réflexion totale contre l'air.

Nous avons anodisé une structure multicouche permettant un guidage pour $\lambda_{0} \simeq 0,75 \mu \mathrm{m}$ (pour $\theta_{\mathrm{L}} \simeq 75^{\circ}$ ), où se situe le maximum de l'émission du matériau. La gamme de densités de courant utilisées ${ }^{7}$ permet un contraste d'indice de 1,55 contre 1,2 , ce qui est suffisant. Nous avons limité l'empilement à 4,5 paires de couches pour des raisons de tenue mécanique. Nous rappelons en outre que la structure a subi une oxydation anodique, afin de stabiliser les propriétés d'émission du matériau (cf. § 1.4., Chap. 4) ; l'autre conséquence est une diminution de l'absorption du matériau, car une part notable de silicium a été transformée en silice.

Le profil d'indice obtenu ${ }^{8}$, déterminé précisément par l'ajustement théorique du spectre de réflectivité mesuré en incidence normale, est donné sur la figure 40. Nous notons $\Lambda$ la période de l'empilement, soit $\Lambda=e_{\mathrm{H}}+e_{\mathrm{L}}$.

6. Cette expression n'est applicable en toute rigueur qu'à une microcavité symétrique ; ici, la réflexion totale donne lieu à un déphasage différant de celui d'une réflexion de Bragg, mais il s'agit dans le cas présent d'une correction faible.

7. $j_{\mathrm{c}}=j_{\mathrm{L}}=333 \mathrm{~mA} / \mathrm{cm}^{2}$, porosité $72 \%$ et $j_{\mathrm{H}}=16,6 \mathrm{~mA} / \mathrm{cm}^{2}$, porosité $58 \%$,

8. $n_{\mathrm{H}}=1,55, e_{\mathrm{H}}=0,186 \mu \mathrm{m}, n_{\mathrm{L}}=1,20, e_{\mathrm{L}}=0,576 \mu \mathrm{m}, n_{\mathrm{c}}=n_{\mathrm{L}}, e_{\mathrm{C}}=2 e_{\mathrm{L}}$, et $n_{\mathrm{sub}}=3,5$. 


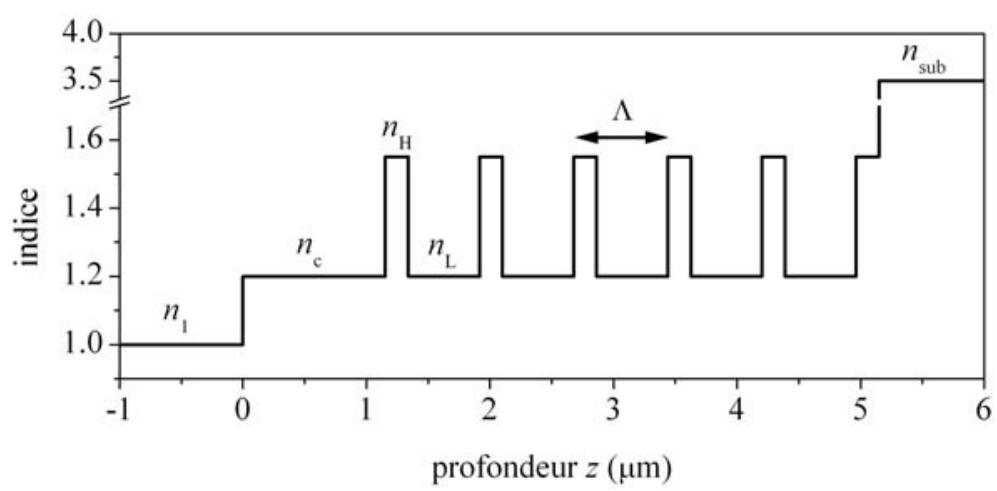

Figure 40. Profil d'indice du guide d'onde. Grâce à la bande interdite photonique de l'empilement périodique, la lumière est guidée dans la couche d'indice $n_{\mathrm{c}}$.

[Index profile of the waveguide. Thanks to the photonic bandgap of the periodic stacking, light is guided in the layer of index $n_{\mathrm{c}}$.]

\subsection{Calcul des modes guidés}

\section{Bande interdite de l'empilement périodique}

Nous considérons dans un premier temps la bande interdite photonique de l'empilement périodique, dans sa version infinie. La structure de bande a été calculée au moyen d'un programme du domaine public ${ }^{9}$, basé sur une méthode d'ondes planes [95].

La figure 41 représente pour les deux polarisations la structure de bande projetée obtenue en traçant, pour chaque valeur de $\beta$, les fréquences accessibles pour toutes les valeurs que peut prendre $k_{z}$ à l'intérieur de la demi-zone de Brillouin, soit entre 0 et $\pi / \Lambda$. Sur la figure finale, les zones grisées représentent le continuum de fréquences accessibles, tandis que les zones restées blanches représentent des fréquences dépourvues de mode électromagnétique.

La réflectivité absolue, mesurée sur l'échantillon en incidence normale, est également reportée en face des diagrammes de bandes. Les fréquences interdites à $\beta=0$ se manifestent par une augmentation notable de la réflectivité, en dépit du faible nombre de couches que comprend notre empilement.

Comme tout système unidimensionnel, le nôtre ne possède pas de bande interdite complète : quelle que soit la fréquence, la figure montre que la propagation interdite se limite à des valeurs de $\beta$ cantonnées dans des intervalles finis. Dans ces conditions, le guidage n'est possible que si ces mêmes valeurs permettent en outre une propagation dans la cavité et une réflexion totale contre l'interface au contact de l'air. Cette dernière condition, qui s'écrit

$$
k_{0}<\beta<k_{0} n_{\mathrm{c}}
$$

9. S.G. Johnson et J.D. Joannopoulos, The MIT Photonic-Bands Package, disponible sur http://ab-initio.mit.edu/mpb/. 


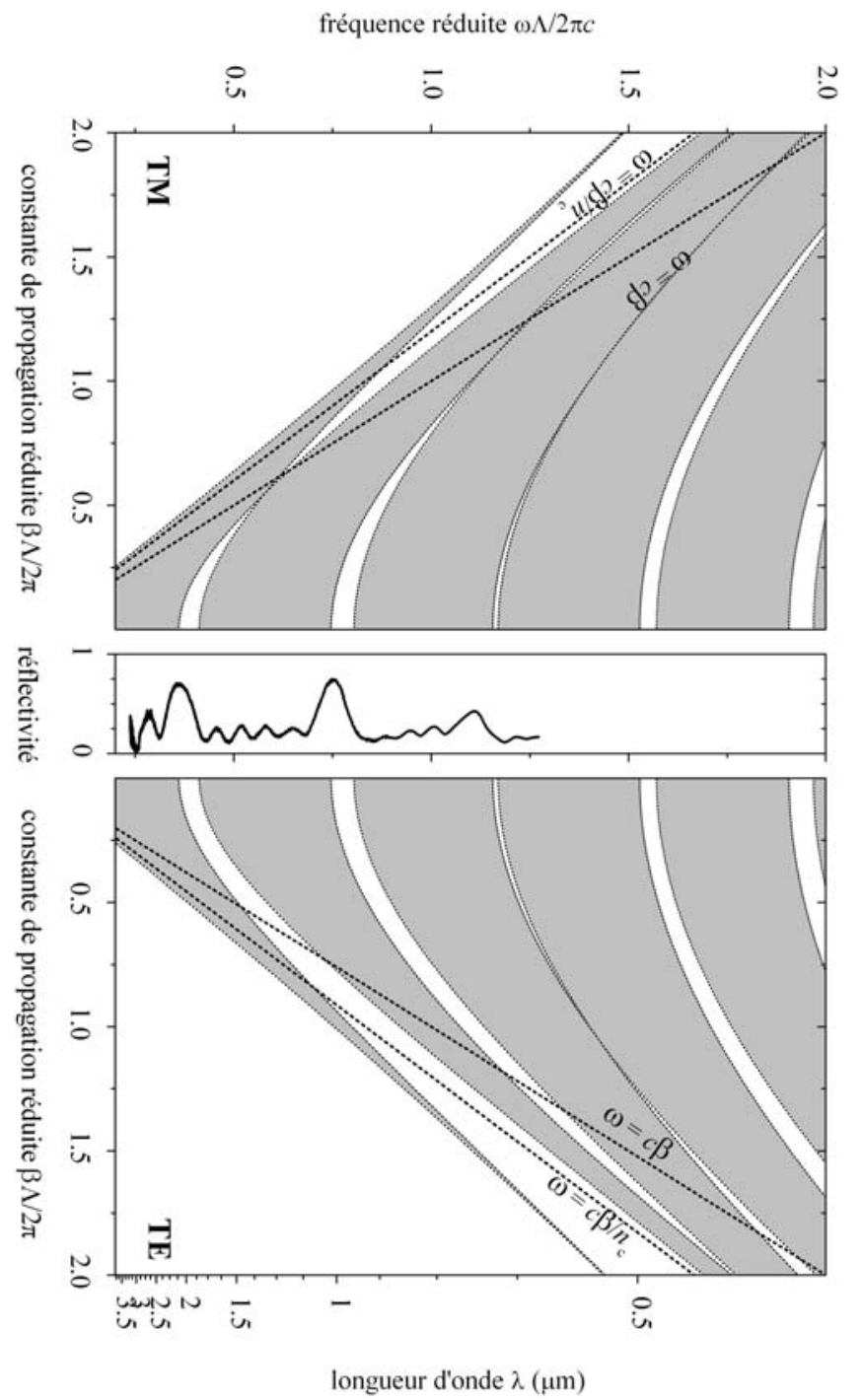

Figure 41. Structure de bande projetée, calculée pour l'empilement infini. Les régions laissées en blanc correspondent à une propagation interdite. Le spectre de réflectivité de l'échantillon, mesuré en incidence normale, est reporté au centre. Les droites $\omega=c \beta$ et $\omega=c \beta / n_{c}$ délimitent les valeurs de $\beta$ permettant une propagation dans la cavité et une réflexion totale contre l'interface supérieure.

[Projected band structure, calculated for the infinite stacking. White regions correspond to forbidden propagation. The experimental reflectance spectrum, measured at normal incidence, is plotted in the center. The lines $\omega=c \beta$ and $\omega=c \beta / n_{\mathrm{c}}$ the range of $\beta$ that allow a propagation in the cavity, and a total internal reflection against the top interface.] 
est matérialisée sur la figure 41 par la zone comprise entre les droites $\omega=c \beta$ et $\omega=c \beta / n_{\mathrm{c}}$. On peut ainsi distinguer graphiquement les fréquences pour lesquelles les conditions de réflexion, nécessaires au guidage, sont vérifiées.

On voit sur la figure que la coexistence de la réflexion totale et de la réflexion de Bragg est possible pour $\lambda \simeq 1,1 \mu \mathrm{m}$ (TE). Pour toutes les autres fréquences, le recouvrement existe mais il n'est pas total : pour $\beta$ vérifiant l'équation (6.15), certaines valeurs de $\beta$ correspondront à un guidage efficace; pour d'autres, l'onde traversera allègrement le miroir de Bragg. En polarisation TM, à cause de la proximité de l'incidence de Brewster (la droite correspondante serait très proche de la droite $\omega=c \beta$ ) qui donne des bandes interdites étroites, le recouvrement est très faible.

\section{Structure complète}

À ce stade, la mise en évidence du mode guidé et l'étude de sa dispersion nécessitent un calcul sur structure réelle. Le diagramme de bandes a donc été calculé en prenant en compte le nombre fini de périodes, la cavité et l'air. Dans la gamme de $\beta$ qui nous intéresse (sous la droite $\omega=c \beta$ ), les bandes interdites de l'empilement fini apparaissent identiques à celles du cas infini. Cet effet n'est pas surprenant, dans la mesure où les grand angles d'incidence associés donnent lieu à des coefficients de réflexion élevés à chaque interface; dans ces conditions, peu de périodes sont requises pour approcher l'efficacité du système infini.

Le seul changement notable concerne l'apparition de modes dans certaines bandes interdites, reportée sur la figure 42. La distribution en profondeur de la puissance transportée dans le plan par ce type de mode, et qui est tracée sur la figure 43, illustre la nature hybride du guidage : onde exponentielle pour $z<0$, en raison de la réflexion totale contre l'interface supérieure, et onde périodique à enveloppe exponentiellement décroissante en raison de la réflexion de Bragg sur le milieu périodique.

Le guidage a été vérifié expérimentalement en mesurant la transmission de lumière blanche à travers un guide de $1,88 \mathrm{~mm}$ de long. Les spectres obtenus, tracés en face des diagrammes de bandes, sur la figure 42 , montrent la grande fenêtre spectrale du guidage, corrélée à la présence du mode dans la bande interdite, tout au moins à faible fréquence. À plus haute fréquence $(\lambda<0,6 \mu \mathrm{m}), \mathrm{c}^{\prime}$ est l'absorption du silicium poreux qui est responsable de la chute d'intensité. On note enfin l'importante disproportion entre les puissances guidées selon les deux polarisation, à l'avantage de la polarisation TE, d'un facteur six.

\section{4. Étude des pertes}

Nous avons pu étudier quantitativement les pertes en propagation guidée, en profitant du confort apporté par la nature émettrice du silicium poreux. 


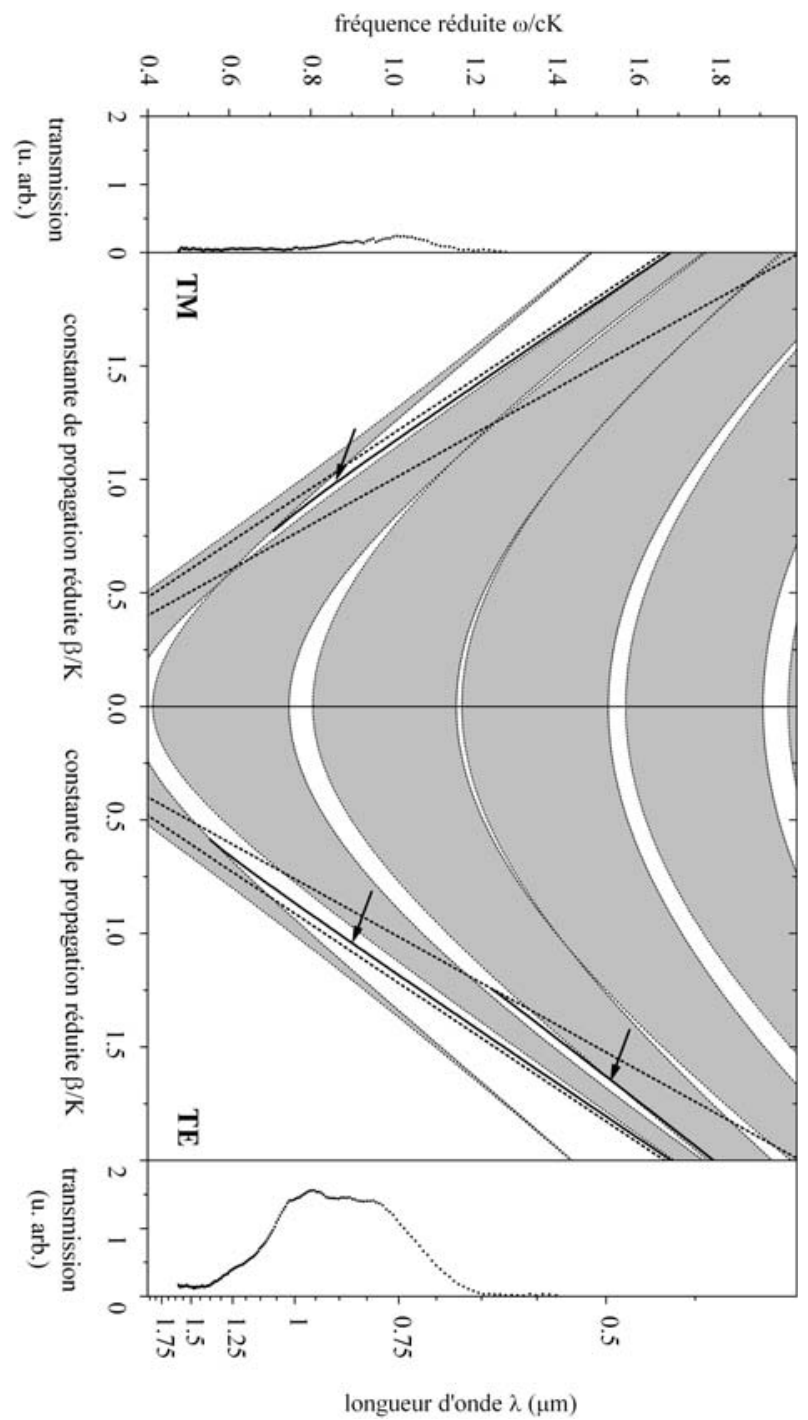

Figure 42. Structure de bande projetée de l'empilement infini, sur laquelle ont été reportés en trait plein les modes (pointés par des flèches) qui apparaissent lorsque le calcul est effectué sur la structure réelle (empilement fini, la cavité, et l'air). En haut et en bas sont reportés (avec la même échelle d'intensité) les spectres de transmission mesurés en lumière blanche sur un guide de 1,88 $\mathrm{mm}$ de long.

[Projected band structure of the infinite stacking, on which it has been reported in thick lines the modes (indicated by arrows) that appear when computing the real structure (finite stacking, cavity, and air). On top and bottom are plotted (using the same scale) the transmittance spectra measured using white light on a waveguide of length $1.88 \mathrm{~mm}$.] 


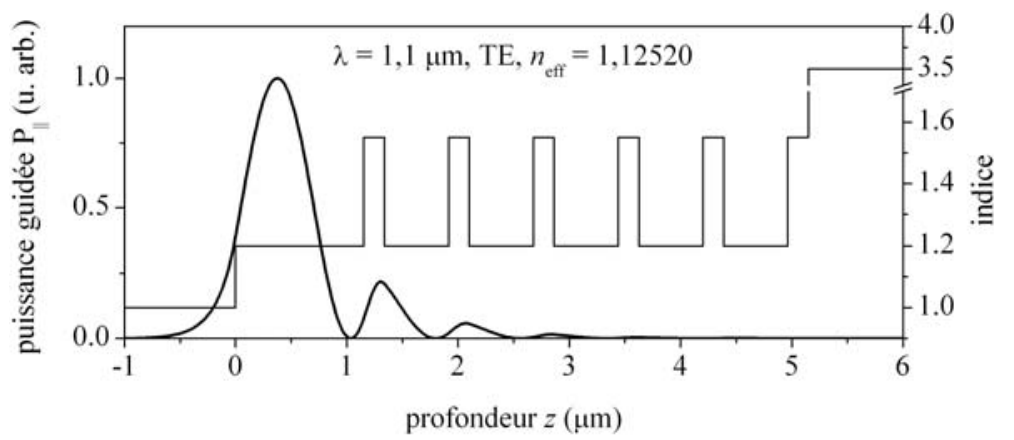

Figure 43. Distribution en profondeur de la puissance guidée à $\lambda=1,1 \mu \mathrm{m}$, en polarisation TE.

[Distribution in depth of the guided power at $\lambda=1.1 \mu \mathrm{m}$, for the TE polarization.]

\section{Principe}

La photoluminescence a été excitée optiquement à la surface de la structure guidante, à une distance $L$ de la tranche de sortie, comme détaillé en $\S 3.3$., chapitre 4 , en choisissant une longueur d'onde fortement absorbée $(\lambda=0,4579 \mu \mathrm{m})$, afin de restreindre l'excitation, et donc l'émission, à la couche supérieure.

\section{Mesure des pertes}

La photoluminescence émise par la tranche a été mesurée pour différentes valeurs de $L$ entre 0 et 1,2 $\mathrm{mm}$. L'atténuation mesurée a été corrigée des effets de la divergence du faisceau, comme nous le détaillons dans l'annexe B. La série de spectres obtenus est représentée sur la figure 44a dans le cas de la polarisation TE, sachant que la polarisation TM donne des spectres d'allure similaire. Pour chaque longueur d'onde, la chute d'intensité en fonction de $L$ est décrite par un déclin exponentiel, comme l'illustre l'insert sur la même figure. Les coefficients d'atténuation obtenus ainsi avec une grande précision sont reportés pour les deux polarisations sur la figure 44b.

\section{Interprétation}

Les pertes mesurées se distinguent des valeurs obtenues sur le guide à fort indice à la fois par leur valeur, mais aussi par la disparité qui existe entre les deux polarisations, la polarisation TM étant notablement handicapée.

Absorption. À faible longueur d'onde, le bénéfice lié au guidage dans une couche de faible indice, mais surtout oxydée, est évident. Dans le rouge visible, les valeurs aussi faibles la dizaine de $\mathrm{cm}^{-1}$ ne sont tout bonnement pas envisageables avec du silicium poreux non-oxydé ${ }^{10}$. En outre, si elles n'atteignent pas les pertes

10. Pour $\lambda=0,7 \mu \mathrm{m}$, une absorption de $200 \mathrm{~cm}^{-1}$ constitue un minimum [54]. 
longueur d'onde $\lambda(\mu \mathrm{m})$
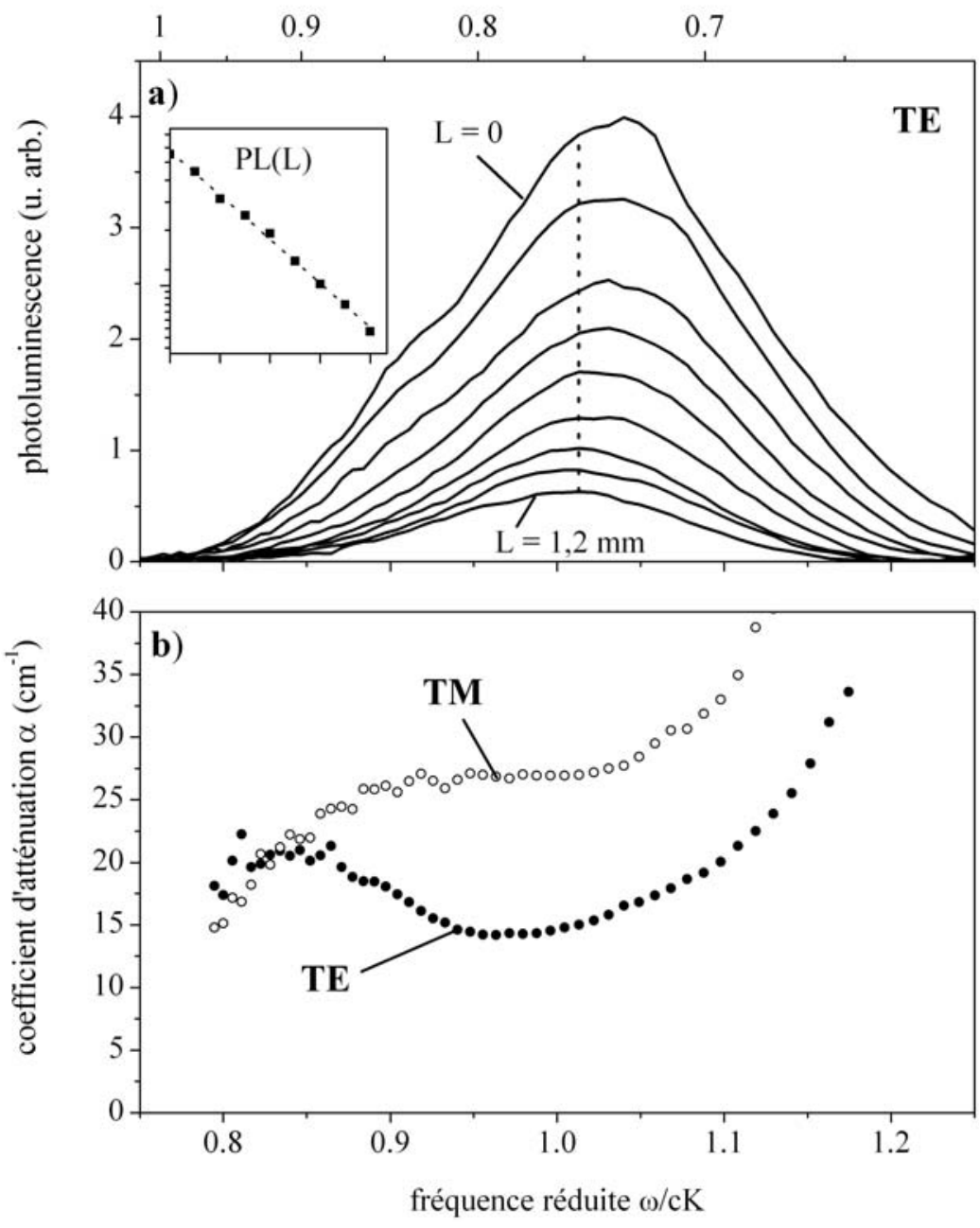

Figure 44. (a) Série de spectres de photoluminescence émise par la tranche, mesurés en polarisation TE pour différentes distances de propagation $L$. L'insert illustre la chute d'intensité (échelle logarithmique) en fonction de $L$, obtenue pour chaque longueur d'onde (ici matérialisée par une ligne verticale en pointillés), à partir de laquelle l'ajustement d'un déclin exponentiel permet de déduire le coefficient d'atténuation. (b) Coefficients d'atténuations obtenus pour les deux polarisations.

[(a) Set of photoluminescence spectra (collected at the edge), measured in TE polarization for several propagation lengths $L$. The inset illustrates the decay of intensity (log scale) vs. $L$, obtained at each wavelength (here indicated by a vertical dotted line), that gives (by fitting) the attenuation coefficient. (b) Attenuations coefficients obtained for both polarizations.] 
record mesurées sur des guides d'onde à base de silicium poreux totalement oxydé et densifié (une fraction de $\mathrm{cm}^{-1}$ à $\lambda=0,6328 \mu \mathrm{m}$ ) [96], elles présentent néanmoins l'avantage de préserver la nature émettrice du matériau.

Diffusions. Au vu de l'équation (6.2), la diffusion Rayleigh doit diminuer avec le contraste d'indice à l'échelle microscopique (même s'il reste du silicium, comme l'atteste la luminescence de la structure), aboutissant à une influence minime. Concernant la diffusion de surface, la rugosité est quasi-inexistante au fond de la couche guidante, car celle-ci a été anodisée avec un fort courant [66]. Qui plus est, cette interface est le lieu d'un champ relativement faible (au moins en polarisation TE), comme le montre la figure 43. Quant aux couches anodisées à fort courant, leur rugosité est limitée en raison de leur épaisseur réduite [14].

Fuites. L'atténuation liée aux fuites a été calculée, et sa dépendance en fonction de la longueur d'onde est reportée pour les deux polarisations sur la figure 45 . L'influence que leur attribue le calcul est négligeable, bien qu'elles ne semblent pas étrangères à la disparité constatée expérimentalement entre les deux mesures.

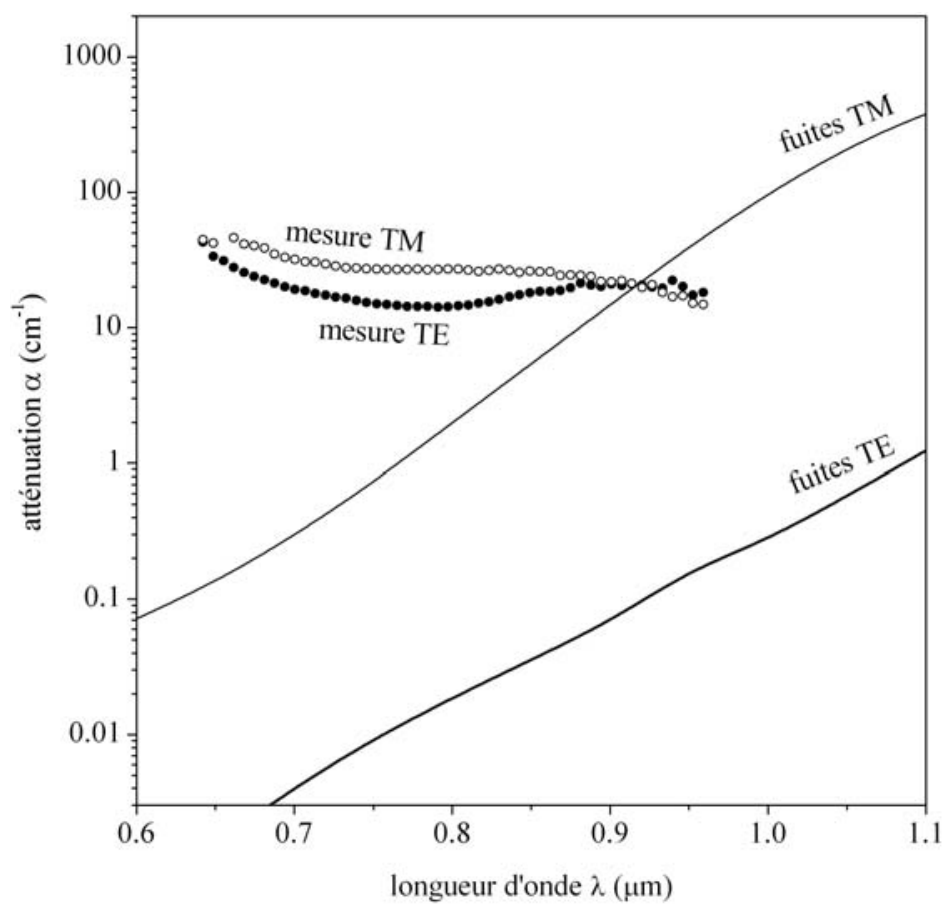

Figure 45. Comparaison de l'atténuation calculée due aux fuites avec les pertes mesurées. [Comparison between the calculated attenuation (due to the leakage) and measurements.] 


\subsection{Autres modes guidés}

La structure considérée est susceptible de donner lieu à d'autres types de modes guidés, bien que leur présence ne remette pas en cause les mesures effectuées.

\section{Modes de fuites}

Les modes dits de fuite (les leaky modes en anglais) ${ }^{11}$ sont des modes parasites, omniprésents dans les structures résonantes à base de miroirs de Bragg, résultant des réflexions sur les lobes secondaires présents de part et d'autre du plateau de haute réflectivité de tout miroir de Bragg. Cette appellation leur est donnée parce qu'ils drainent «efficacement » l'énergie hors du guide, vers le substrat.

Dans le diagramme de bande, ils sont localisés entre les droites $\omega=c \beta$ et $\omega=$ $c \beta / n_{\mathrm{c}}$, dans les régions grisées. Ils sont caractérisés par des pertes colossales, et à ce titre, ils sont à inclure dans l'ensemble des modes rayonnants, qui n'interviennent pas dans nos mesures, étant donné les longueurs sondées.

\section{Modes du miroir de Bragg}

Les couches de fort indice appartenant au miroir de Bragg forment un ensemble couplé de guides d'onde à saut d'indice [4]. Les modes associés (cinq dans le cas présent) sont fortement fuyants en raison de la proximité immédiate du substrat. Nous ne les avons pas considérés puisqu'ils ne peuvent être excités par la source lumineuse, qui est localisée dans un milieu d'indice inférieur ${ }^{12}$.

En définitive, le guidage de la lumière par un effet de bande interdite photonique semble parfaitement adapté aux spécificités du silicium poreux, même si l'obtention d'un contraste d'indice plus élevé dans l'empilement périodique constituerait une amélioration dont la fenêtre spectrale de guidage efficace pourrait tirer parti.

Outre la diminution des pertes et la facilité de couplage ${ }^{13}$ liées à la propagation dans un milieu de faible indice, on notera que la réflexion de Bragg permet de s'affranchir de la systématique dispersion des polarisations qui fait que $\beta_{\mathrm{TE}}>\beta_{\mathrm{TM}}$ (l'effet est dû à des phases différentes lors de la réflexion totale). Dans le cas d'un miroir de Bragg, le déphasage subi par l'onde réfléchie peut se contrôler, en jouant sur les indices et épaisseurs des couches qui composent l'empilement. On peut donc envisager d'obtenir des valeurs identiques, voire d'inverser la tendance ( $c f$. la discussion page 52).

11. Si tous les modes guidés que nous avons étudiés sont, en toute rigueur, à fuite, les modes de fuite regroupent ceux dont l'existence même n'est pas souhaitée.

12. Dans la mesure où leur profondeur les met à l'abri d'une excitation efficace, nous pouvons faire abstraction des émetteurs les plus proches du miroir de Bragg, dont l'émission en champ proche aurait pu donner un rayonnement dans les couches de fort indice.

13. Dans la mesure où nous vivons dans un monde à faible indice, le faible indice du guide est un plus indéniable, offrant une transition entre ces deux mondes moins douloureuse : le couplage par la tranche est plus efficace en raison des réflexions contre l'interface qui sont diminuées et de l'épaisseur plus importante; le couplage par un réseau s'accommode d'une période plus grande ; enfin, on peut se contenter d'un prisme de couplage d'indice plus faible. 
Dans ce chapitre, nous nous sommes intéressé à des structures planaires guidantes. Nous avons passé en revue les causes de pertes les plus fréquentes, puis proposé une approche théorique, en s'appuyant sur le formalisme des matrices de transfert, permettant de calculer l'atténuation de la puissance transportée dans le plan, ce pour des structures arbitraires.

Nous avons réalisé et caractérisé quantitativement deux types de structures guidantes à base de silicium poreux : un guide d'onde à saut d'indice, et un guide d'onde à faible indice, exploitant un effet de bande interdite pour le transport de lumière.

Dans les deux cas, nous avons obtenu des structures aptes à un guidage efficace dans le proche infrarouge, condition requise pour l'étude de l'effet d'une structuration périodique de l'indice dans le plan. 


\section{Contrôle de la propagation guidée par une structuration périodique de l'indice dans le plan}

Nous sommes maintenant en mesure d'étudier l'influence d'une structuration périodique de l'indice dans le plan de la propagation guidée. Après une brève introduction à la méthode de modes couplés, nous présentons les mesures réalisées de façon «passive », par transmission de lumière blanche. Les résultats obtenus sont confrontés à la théorie, et l'effet de la structuration en termes d'émission de surface est discuté.

\section{Principe}

Les propriétés optiques d'un guide que l'on rend périodique peuvent être appréhendées de façon particulièrement intuitive si l'on raisonne en termes de perturbation.

Nous l'avons vu en $\S 3.3$., chapitre 2, les modes d'un guide possèdent une dispersion $\omega(\mathbf{k})$ d'allure relativement simple, comme l'illustre la figure 46a, l'allure symétrique du graphe dénotant la possibilité des deux sens de propagation. Nous distinguerons dorénavant par un indice négatif les modes se propageant dans le sens des $x$ négatifs. Dit autrement, $\beta_{-m}=-\beta_{m}$.

L'introduction d'une perturbation d'indice $\Delta n(x, z)$, de période $\Lambda$ selon $x$ (Fig. 46b), impose aux vecteurs d'onde d'appartenir à un réseau de période $K=2 \pi / \Lambda$, et rend possible le couplage entre deux modes se propageant en sens inverse, pour peu que la condition ${ }^{1}$

$$
\beta_{l}-\beta_{-m} \simeq p K,
$$

soit satisfaite pour un entier $p$. Sur la figure $46 \mathrm{~b}$, la périodicité du réseau réciproque est prise en compte en reportant les courbes de dispersion avec la période $K$, et l'équation (7.1) est vérifiée à chaque intersection des courbes de dispersion.

1. Nous conservons ici la notation $\beta$, mais il est évident qu'elle désigne maintenant uniquement la composante $k_{x}$, l'invariance selon toutes les directions du plan ayant été brisée par l'introduction de la perturbation. 

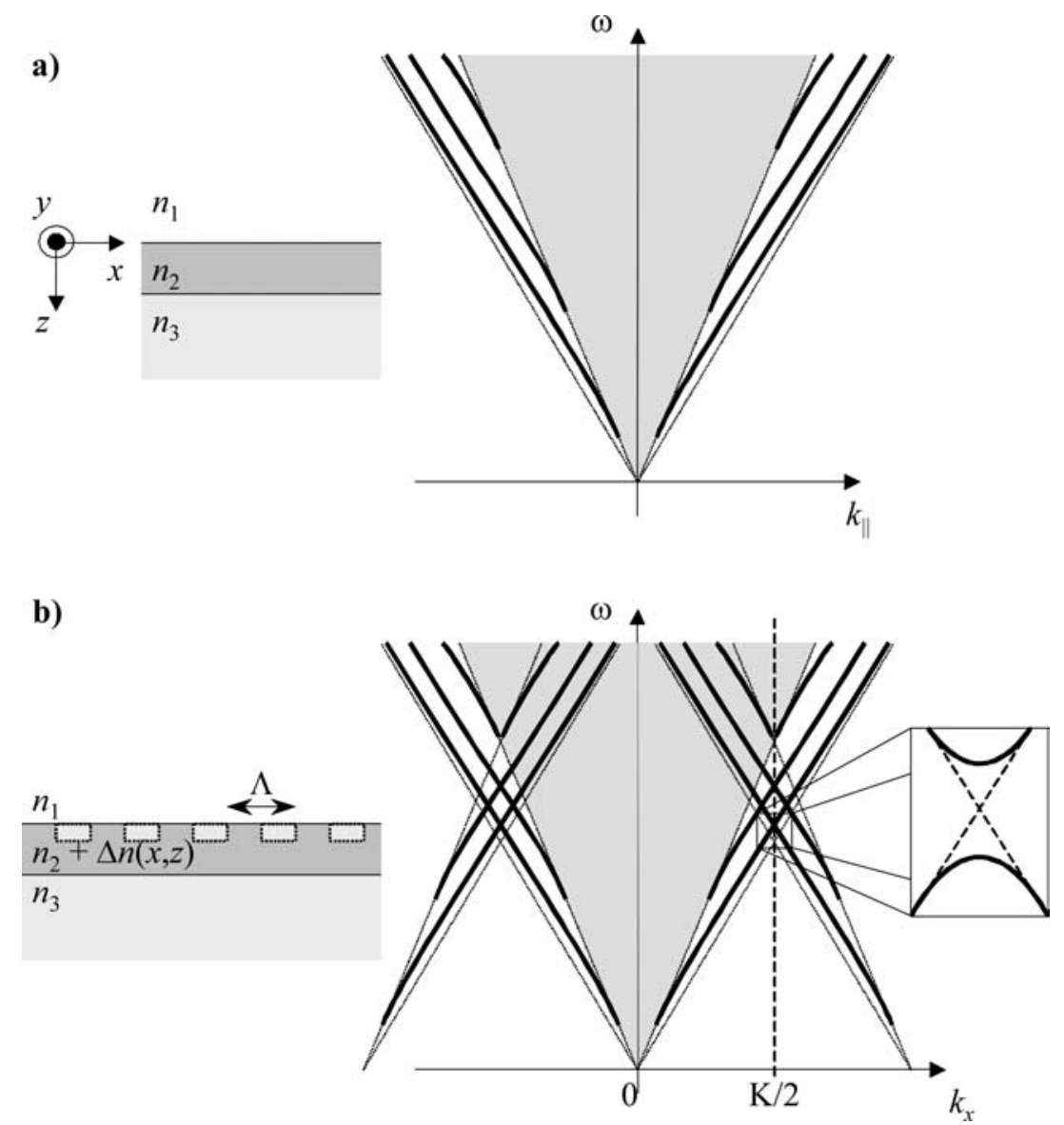

Figure 46. (a) Guide planaire homogène et dispersion associée. La région grisée représente le continuum de modes rayonnants. (b) Une perturbation d'indice $\Delta n(x, z)$, de période $\Lambda$ selon $x$, est introduite. Si l'on reproduit les courbes de dispersion avec une période $K=2 \pi / \Lambda$, chaque intersection indique une possibilité de couplage entre deux modes se propageant en sens inverse. Si le couplage est efficace, la dégénérescence est levée, et une bande interdite apparaît, comme l'illustre le détail agrandi. En raison des symétries des courbes de dispersion, on peut en outre se restreindre aux vecteurs d'onde appartenant à la demie zone de Brillouin $\left(0 \leq k_{x} \leq K / 2\right)$.

[(a) Homogeneous planar waveguide and related dispersion. The grey region represents the continuum of radiated modes. (b) A perturbation $\Delta n(x, z)$ (of period $\Lambda$ ) of the optical index is introduced. By duplicating the dispersion curves with a period $K=2 \pi / \Lambda$, each crossing indicates the opportunity for two modes to be coupled together. In case of efficient coupling, the degeneracy can be lifted and a bandgap can appear, as illustrated in the inset. Due to the symmetry, the dispersion curves can be restricted to the first half Brillouin zone $\left(0 \leq k_{x} \leq K / 2\right)$.] 
Un couplage efficace permet une levée de la dégénérescence, et l'apparition d'une bande interdite, comme l'illustre le détail, sachant que la largeur de la bande interdite dépend directement de l'efficacité du couplage. Bon nombre d'intersections sont localisées au bord de la demi-zone de Brillouin $\left(k_{x}=K / 2\right)$, elles indiquent alors des couplages que nous appelons diagonaux, entre un mode et son homologue de sens contraire $(l=m)$, à distinguer des couplages non diagonaux $(l \neq m)$, pour lesquels $k_{x}<K / 2$.

Si le couplage est faible, cette description en termes de perturbation est satisfaisante, et le calcul des modes est considérablement simplifié car il se réduit au calcul des modes non-perturbés, puis de l'efficacité du couplage introduit par la perturbation.

\section{Méthode des modes couplés}

Cette méthode perturbative convient généralement bien aux structures guidantes périodiques, même si sa généralité permet de l'appliquer à des perturbation d'autre nature (modulations acousto-optique, électro-optique, etc.). Le lecteur intéressé se reportera avec profit à l'article original [97] (pour la polarisation TM, cf. [98]), ou à l'ouvrage de Yariv [84] pour un exposé plus détaillé. Nous donnons ici en polarisation TE les étapes clés de la méthode dont nous avons élargi le domaine d'application aux couplages non diagonaux.

\subsection{Guide non perturbé}

Le système non perturbé est un guide planaire, tel qu'il est représenté sur la figure 46a : les indices sont supposés réels $\left(n_{2}>n_{3} \geq n_{1}\right)$, et les milieux de part et d'autre de la couche guidante semi-infinis. Ces conditions éliminant la possibilité de pertes, les modes guidés susceptibles d'exister sont parfaits. Ils constituent les solutions discrètes de l'équation d'onde

$$
\nabla^{2} \mathbf{E}-\mu_{0} \epsilon_{0} \epsilon(\mathbf{r}) \frac{\partial^{2} \mathbf{E}}{\partial t^{2}}=0
$$

En polarisation TE, chaque mode guidé $m$ est décrit par un champ électrique dont la seule composante non nulle, selon $y$, s'écrit

$$
E_{y}^{(m)}(x, z, t)=E_{y}^{(m)}(z) \mathrm{e}^{+\mathrm{i} \beta_{m} x-\mathrm{i} \omega t} .
$$

\subsection{Guide perturbé}

\section{Perturbation}

L'équation d'onde du guide perturbé s'écrit

$$
\nabla^{2} \mathbf{E}-\mu_{0} \epsilon_{0} \epsilon(\mathbf{r}) \frac{\partial^{2} \mathbf{E}}{\partial t^{2}}=\mu_{0} \frac{\partial^{2} \mathbf{P}_{\text {pert }}}{\partial t^{2}}
$$


où $\mathbf{P}_{\text {pert }}(x, z)$ est la polarisation induite par la perturbation. Si l'on ignore la possibilité de couplage avec le continuum de modes rayonnants, on peut développer la composante $E_{y}$ sur la base des modes discrets $E_{y}^{(m)}(z)$ du guide non perturbé, selon

$$
E_{y}(x, z, t)=\frac{1}{2} \sum_{m} a_{m}(x) E_{y}^{(m)}(z) \mathrm{e}^{\mathrm{i} \beta_{m} x-\mathrm{i} \omega t}+\mathrm{cc} .
$$

\section{Couplage}

Si l'on injecte la décomposition de l'équation (7.5) dans l'équation d'onde du guide perturbé (Éq. (7.4)), si l'on multiplie par $E_{y}^{(l)}(z)$ et que l'on intègre pour $z$ variant de $-\infty$ à $+\infty$, si l'on suppose que les variations des amplitudes $a_{m}$ sont lentes, on obtient que

$$
\frac{\mathrm{d} a_{-l}}{\mathrm{~d} x} \mathrm{e}^{+\mathrm{i} \beta_{-l} x-\mathrm{i} \omega t}-\frac{\mathrm{d} a_{+l}}{\mathrm{~d} x} \mathrm{e}^{+\mathrm{i} \beta_{l} x-\mathrm{i} \omega t}-\mathrm{cc}=\frac{\mathrm{i}}{2 \omega} \frac{\partial^{2}}{\partial t^{2}} \int_{-\infty}^{+\infty}\left[P_{\text {pert }}(\mathbf{r}, t)\right]_{y} E_{y}^{(l)}(z) \mathrm{d} z .
$$

Cette dernière équation, où apparaissent à la fois des composantes du champ se propageant dans les sens $+x$ et $-x$, montre que la perturbation est susceptible $\mathrm{d}^{\prime}$ induire une modification longitudinale des amplitudes des modes, tout au moins si son influence reste la même suffisamment longtemps. Ce dernier point impose que le membre de droite de l'équation (7.6) trouve à gauche un terme avec lequel il y ait accord de phase. C'est à ce stade qu'intervient de façon sélective le caractère périodique de la perturbation.

Notre perturbation $\mathbf{P}_{\text {pert }}$ est créée par des variations locales de l'indice, selon

$$
\mathbf{P}_{\text {pert }}=\Delta n^{2}(\mathbf{r}) \epsilon_{0} \mathbf{E}(\mathbf{r}, t)
$$

Sa nature périodique, de période $\Lambda$ selon $x$, permet de développer $\Delta n^{2}$ comme

$$
\Delta n^{2}(x, z)=\Delta n^{2}(z) \sum_{q=-\infty}^{\infty} u_{q}(z) \exp (\mathrm{i} q K x),
$$

où $K=2 \pi / \Lambda$, comme précédemment. En particulier, s'il existe une harmonique $p$ telle que

$$
\beta_{l}-\beta_{-m} \simeq p K
$$

alors chacun des deux terme à gauche dans l'équation (7.6) trouve dans la partie droite un terme avec lequel il est en accord de phase, et le couplage se trouve régi par le système

$$
\left\{\begin{array}{l}
\frac{\mathrm{d} a_{-l}}{\mathrm{~d} x}=\kappa_{p, l m} a_{+m} \mathrm{e}^{+\mathrm{i} 2(\Delta \beta) x} \\
\frac{\mathrm{d} a_{+m}}{\mathrm{~d} x}=\kappa_{p, l m}^{*} a_{-l} \mathrm{e}^{-\mathrm{i} 2(\Delta \beta) x}
\end{array}\right.
$$


où $\Delta \beta \equiv\left(\beta_{+l}-\beta_{-m}\right) / 2-p K / 2$ définit l'écart à la condition (7.9), et où le coefficient de couplage $\kappa_{p, l m}$ est donné par $^{2}$

$$
\kappa_{p, l m}^{(\mathrm{TE})}=\frac{-\mathrm{i} \omega \epsilon_{0} u_{p}}{4} \int_{-\infty}^{+\infty} \Delta n^{2}(z) E_{y}^{(l)}(z) E_{y}^{(m)}(z) \mathrm{d} z .
$$

On retiendra de cette expression relativement simple que le coefficient de couplage témoigne du recouvrement de trois distributions : les distributions de champ associées aux deux modes non perturbés, et la perturbation d'indice.

\subsection{Solutions couplées}

Pour une perturbation appliquée sur une longueur finie, on montre que le mode $\tilde{\beta}_{l}$, variante perturbée du mode $\beta_{+l}$, est tel que

$$
\tilde{\beta}_{+l}=\beta_{0} \pm \mathrm{i} \sqrt{\left|\kappa_{p, l m}\right|^{2}-\left(\beta_{+l}-\beta_{0}\right)^{2}}=\beta_{0} \pm \mathrm{i} S,
$$

où $\beta_{0}=p K / 2-\left(\beta_{+l}+\beta_{-m}\right) / 2$.

La dispersion du mode perturbé est illustrée sur la figure 47 : pour les fréquences pour lesquelles le couplage est négligeable $\left(\kappa_{p, l m} \ll \beta-\beta_{0}\right), \tilde{\beta}_{+l} \simeq \beta_{+l}$. Lorsque le couplage devient assez fort, tant que $\kappa_{p, l m} \leq \beta_{+l}-\beta_{0}, \tilde{\beta}_{+l}$ s'écarte de $\beta_{+l}$. Dès que $\kappa_{p, l m}>\beta_{+l}-\beta_{0}$, on se trouve alors dans la bande interdite, et la constante de propagation perturbée prend une partie imaginaire non nulle, synonyme d'atténuation de la puissance transportée par le mode. L'atténuation la plus forte a lieu pour $\beta_{+l}=\beta_{0}$, et son coefficient est directement donné par $\kappa_{p, l m}$.

\subsection{Coefficient de transmission}

Lorsque la perturbation s'étend longitudinalement sur une longueur $L$, on montre que le coefficient de transmission du mode $l$, lorsqu'il est couplé avec le mode $m$, s'écrit

$$
T_{l m}=\left|\frac{a_{+l}(L)}{a_{+l}(0)}\right|^{2}=\frac{2|S L|^{2}}{(\Delta \beta L)^{2} f_{-}(S L)+|S L|^{2} f_{+}(S L)},
$$

où nous avons défini la fonction complexe $f_{ \pm}(z) \equiv \cosh [2 \operatorname{Re}(z)] \pm \cos [2 \operatorname{Im}(z)]$.

\subsection{Cas de la polarisation TM}

Le calcul en polarisation TM suit la même démarche, à ceci près que c'est alors le champ magnétique $\mathbf{H}$ qui est développé sur les modes $H_{y}^{(m)}$ du guide. L'expression du coefficient de couplage diffère sensiblement :

$$
\kappa_{p, l m}^{(\mathrm{TM})}=\frac{\mathrm{i} \omega \mu_{0} u_{p}}{4} \int_{-\infty}^{+\infty} \frac{\Delta n^{2}(z)}{n^{2}(z)} H_{y}^{(l)}(z) H_{y}^{(m)}(z) \mathrm{d} z .
$$

2. Toute ressemblance entre cette manipulation algébrique et un calcul d'élément de matrice de mécanique quantique n'est pas fortuite... 


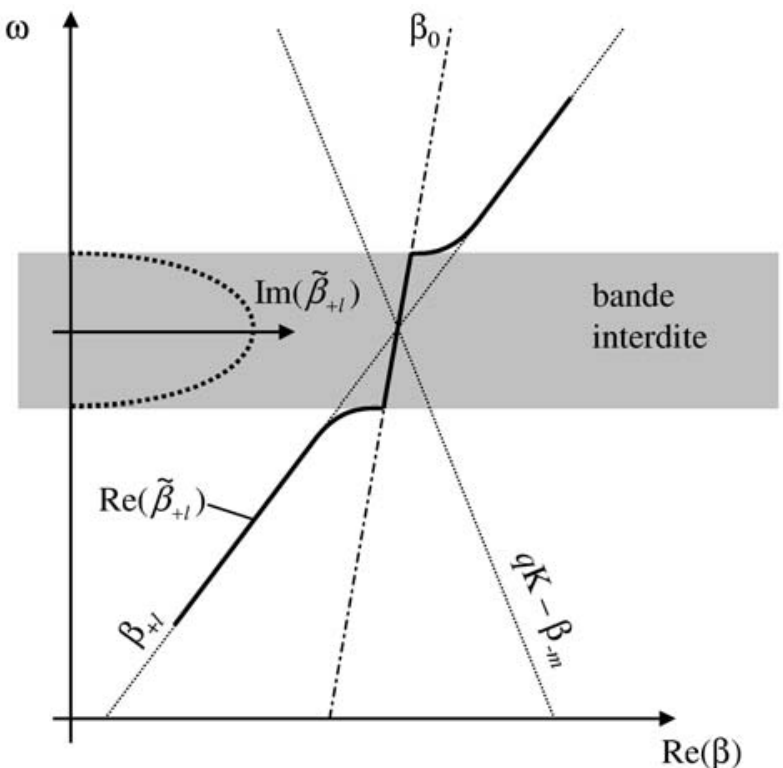

Figure 47. Illustration du couplage du mode $\beta_{+l}$ avec le mode $\beta_{-m}$. Le tracé en trait épais représente la dispersion du mode $\tilde{\beta}_{+l}$ résultant. Dans la bande interdite, la partie imaginaire de $\tilde{\beta}_{+l}$ est reportée en trait épais pointillé, dans une échelle arbitraire. Le mode perturbé $\tilde{\beta}_{-m}$ n'a pas été reporté.

[Illustration of the coupling between $\beta_{+l}$ and $\beta_{-m}$ modes. The plot in thick line represents the dispersion of the resulting $\tilde{\beta}_{+l}$ mode. Within the bandgap, the imaginary part of $\tilde{\beta}_{+l}$ is plotted in thick dotted line, using an arbitrary scale. The perturbed $\tilde{\beta}_{-m}$ mode is not plotted.]

\subsection{Récapitulatif}

En définitive, le calcul des modes couplés se limite au calcul des modes guidés du guide non-perturbé (généralement en résolvant une équation transcendante [84]), et au calcul des coefficients de couplages $\kappa_{p, l m}$ en utilisant soit l'équation (7.11), soit l'équation (7.14), selon la polarisation considérée.

Pour chaque couplage, on est ensuite à même de calculer la transmission du mode perturbé, grâce à l'équation (7.13).

\section{Description de l'échantillon}

\subsection{Cahier des charges}

Nous souhaitons mettre en évidence un effet de bande interdite photonique dans le plan de l'échantillon. Dans ce but, nous avons conjugué deux types de structurations :

- une structuration en profondeur, afin de créer une structure guidante planaire (à deux couches), afin de favoriser un transport lumineux dans le plan; 
- la structuration périodique à étudier, et dont on attend des effets sur la lumière guidée.

En pratique, la difficulté tient principalement à l'incompatibilité entre ces deux points, dans la mesure où la perturbation dans le plan peut être telle que le guidage même peut être remis en question (à cause de la diminution de l'indice moyen de la couche guidante, par exemple).

La fabrication de nos échantillons fait intervenir deux étapes : l'anodisation d'une succession de deux couches, réalisée dans l'obscurité, puis la photodissolution holographique, dans le même électrolyte, mais en l'absence de courant, comme détaillé en $\S 1.3$., chapitre 4 . Si la première étape est bien maîtrisée en ce qui concerne les indices et épaisseurs à attendre, comme l'attestent les résultats présentés dans le précédent chapitre, la deuxième étape demande plus de prudence. Bien que des études aient été menées pour quantifier la diminution d'indice induite par l'illumination [60], l'effet de la période du motif lumineux, ainsi que le profil d'indice obtenu en profondeur restent assez mal connus.

Nous avons dû tester différentes conditions expérimentales, donnant tantôt des guides sans aucun effet de bande interdite (structuration probablement trop faible), tantôt sans guidage du tout. Un bon compromis a pu être obtenu en utilisant des structures légèrement multimodes, donc forcément moins sujettes à la disparition complète du guidage.

\subsection{Conditions de formation}

La structure présentée ici possède deux couches : la couche supérieure, d'épaisseur $e_{2}=1,6 \mu \mathrm{m}$, d'indice $n_{2} \simeq 1,6$, fait office de milieu guidant, et la couche inférieure d'épaisseur $e_{3}=4,5 \mu \mathrm{m}$, d'indice $n_{3}=1,3$ joue le rôle de gaine ${ }^{3}$. L'échantillon a ensuite séjourné pendant $8 \mathrm{~min}$ dans son électrolyte, toute sa surface étant illuminée par une figure d'interférences obtenue au moyen de deux faisceaux de densité de puissance respective $20 \mathrm{~mW} / \mathrm{cm}^{2}$. La période de la structuration a pu être déterminée précisément en mesurant les angles de la diffraction d'un faisceau laser. En l'occurrence ici, $\Lambda=0,45 \mu \mathrm{m}$. Nous avons pris soin d'orienter l'échantillon de telle sorte que les lignes de la structuration soient parallèles aux directions de clivage. Après clivage, notre échantillon a une longueur effective $L=1,3 \mathrm{~mm}$, ce qui permet de sonder près de trois mille périodes.

Nous pouvons compter sur une transparence suffisante dans l'infrarouge, d'autant que l'épaisseur de la gaine nous préserve des fuites vers le substrat.

3. Les conditions d'anodisation sont les suivantes : $j=16,6 \mathrm{~mA} / \mathrm{cm}^{2}$, porosité $58 \%$ pour la couche guidante $; j=50 \mathrm{~mA} / \mathrm{cm}^{2}$, porosité $65 \%$ pour la gaine. 


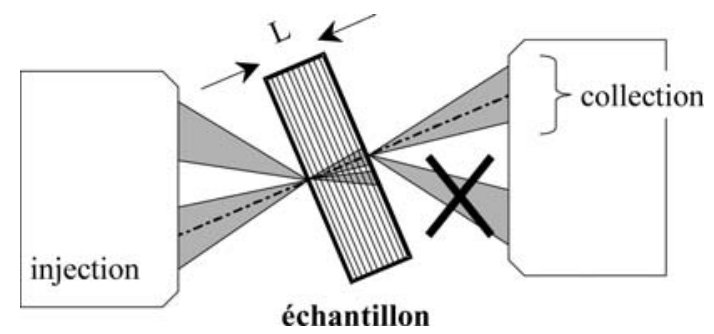

Figure 48. Géométrie de la mesure de transmission sur un guide périodique. Les deux objectifs sont désaxés, et on ne considère que le demi-faisceau qui s'est propagé perpendiculairement aux tranches de l'échantillon.

[Geometry of the transmittance measurement on a periodic waveguide. Both objectives are used off-axis, and one consider only the half-beam that propagates perpendicularly to the sample edges.]

\section{Mesures de transmission}

\subsection{Angles de mesure}

Pour nous affranchir de l'aveuglement axial des objectifs Cassegrain, nous en avons fait un utilisation hors axe, comme l'illustre la figure 48. L'échantillon est pivoté dans son plan de telle sorte que sa tranche d'entrée est attaquée en incidence normale par l'un des demi-faisceaux provenant de l'objectif d'injection (à gauche). L'objectif de collection (à droite) est désaxé, et l'imagerie de la tranche de sortie nous permet de nous restreindre à la portion de faisceau qui s'est propagée perpendiculairement à la structuration. Cette configuration définit l'azimut $\phi=0$.

En outre, cela n'apparaît pas sur le dessin mais les ouvertures des deux objectifs sont en réalité différentes. Au final, le recouvrement des deux ouvertures définit une ouverture effective (dans le plan) de $2^{\circ}$ de demi-angle au sommet.

\subsection{Spectres}

La transmission guidée a été mesurée en lumière blanche pour les deux polarisations. La transmission globale de l'échantillon (donc comparée à une mesure sans échantillon) était typiquement de l'ordre de quelques pour cent. Cette valeur, dans laquelle interviennent les pertes inhérentes au couplage par la tranche, est tout à fait honorable et témoigne de la bonne qualité optique de la structure. Par la suite, pour une caractérisation directe des bandes interdites, les spectres ont été normalisés et tracés sur la figure 49.

Pour les deux polarisations, les spectres laissent apparaître plusieurs gammes spectrales interdites, pour lesquelles la transmission peut chuter de deux décades. En outre, les quatre bandes interdites présentes en polarisation TE entre $\lambda=1,3 \mu \mathrm{m}$ et $\lambda=1,4 \mu \mathrm{m}$ laissent place à une seule bande large en polarisation TM. 

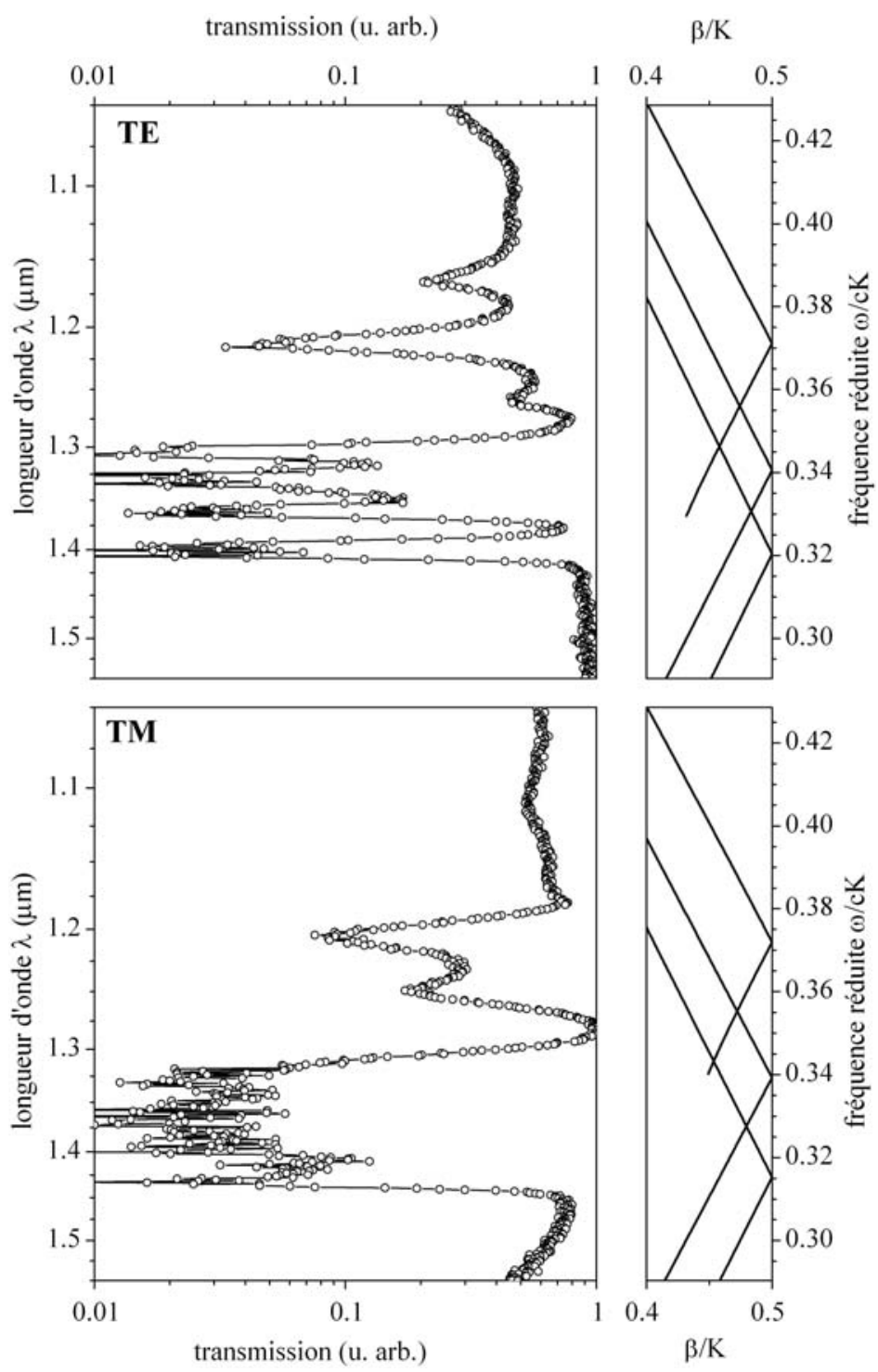

Figure 49. Spectres de transmission mesurés sur le guide périodique, pour les deux polarisations. Sont également représentées à droite les courbes de dispersion de modes non perturbés, simplement repliées pour tenir compte de la périodicité. Chaque intersection correspond à une chute de transmission.

[Transmittance spectra measured on the periodic waveguide, for both polarizations. The dispersion curves of the unperturbed modes are also plotted on the right. They have been folded in order to take into account the periodicity. Each crossing corresponds to a decrease of transmittance.] 
À droite de chaque spectre sur la figure 49, nous avons représenté les courbes de dispersion calculées pour un guide simple, faisant uniquement intervenir la période pour le repliement à $\beta=K / 2$. Ces courbes, obtenues à partir des valeurs $\mathrm{d}^{\prime}$ indices (et d'épaisseur) approximativement connues, en s'autorisant de légers ajustements, montrent que l'on peut associer chaque chute d'intensité à un croisement dans le diagramme de dispersion. Ce résultat incite en outre à attribuer la largeur de la bande TM à un chevauchement de plusieurs bandes.

À ce stade cependant, toute considération de largeur ou d'efficacité des bandes interdites requiert une description précise de la perturbation.

\subsection{Caractérisation de la perturbation}

\section{Modèle}

Dans des cas simples (couche unique sous illumination homogène), il a été démontré que l'efficacité du processus de dissolution était grosso modo proportionnelle à la densité de puissance lumineuse $[58,60]$. Dans nos conditions d'illumination holographique, nous pouvons raisonnablement envisager une perturbation d'indice de dépendance sinusoïdale, de période $\Lambda$ dans le plan, atténuée en profondeur en raison de l'absorption du faisceau d'illumination, et nous l'écrivons donc

$$
\Delta n^{2}(x, z)=\left\{\begin{array}{ll}
0 & z<0 \\
\Delta n_{0}^{2} \cos ^{2}(K x / 2) \exp \left(-\alpha_{\mathrm{ill}} z\right) & z \geq 0
\end{array},\right.
$$

où $\Delta n_{0}^{2}$ quantifie l'amplitude de la perturbation à l'interface supérieure de la couche ( $z=0)$, et $\alpha_{\text {ill }}$ l'atténuation du faisceau d'illumination holographique.

Les seuls coefficients non nuls du développement en série de Fourier introduits dans l'équation (7.8) sont donc $u_{0}=1 / 2$ et $u_{ \pm 1}=\mp \mathrm{i} / 4$. L'amplitude $u_{0} \mathrm{du}$ fondamental n'est pas en mesure de coupler des modes différents, on montre que son influence se limite, pour chaque fréquence, à soustraire à la constante de propagation $\beta_{+l}$ de chaque mode la quantité (réelle et positive) i $\kappa_{0, l l}$. Ce sont les autres harmoniques $u_{ \pm 1}$ qui sont susceptibles de donner lieu à un couplage entre modes de directions opposées.

Nous nous sommes donc attaché à reproduire par la méthode des modes couplés le jeu complet des bandes interdites : pour chaque polarisation, les deux seuls paramètres à ajuster sont $\Delta n_{0}$ et $\alpha_{\text {ill }}$. L'effet du fondamental de la perturbation est pris en compte en calculant $\kappa_{0, l l}$, puis chaque bande interdite est obtenue pour chaque couple $(l, m)$, en calculant le coefficient de couplage $\kappa_{1, l m}$ (Éq. (7.11) ou (7.14)) et la transmission (Éq. (7.13)) associés. Nous verrons par la suite que ces deux paramètres n'ont pas la même influence sur le coefficient de couplage. En particulier, la nature multimode du guide, par les nombreuses couplages qu'elle rend possible, sera un atout décisif pour l'extraction d'un jeu $\left(\Delta n_{0}, \alpha_{\text {ill }}\right)$ unique. 


\section{Anisotropie}

Le traitement séparé des deux polarisations décrit dans le paragraphe précédent est justifié par l'anisotropie reconnue du silicium poreux [49], mais surtout dans le cas présent par une anisotropie inhérente à la structuration holographique. En effet, cette dernière hypothèse est la seule qui permette d'interpréter la largeur des bandes interdites qui distingue les deux polarisations, dans la mesure où le profil des champs associés aux modes, et donc les coefficients de couplages d'après les équations $(7.11,7.14)$, sont peu influencés par la seule polarisation. Polisski et al. ont déjà fait état de tels effets d'anisotropie lorsque, comme dans le cas présent, le faisceau servant à la photodissolution est polarisé [76].

Aussi, nous avons distingué les indices ordinaires $\left(n_{20}\right.$ et $\left.n_{30}\right)$ et extraordinaires $\left(n_{2 \mathrm{e}}\right.$ et $\left.n_{3 \mathrm{e}}\right)$, qui sont respectivement vus par les composantes du champ électrique dans le plan et perpendiculairement à la surface. Dans ces conditions, la polarisation TE ne voit que les indices ordinaires, alors que la polarisation TM voit, en toute rigueur, des indices dont la valeur est comprise entre celle des indices ordinaires et extraordinaires, selon l'incidence. Étant donné que l'incidence des faisceaux dans les conditions de guidage est relativement grande, nous avons supposé que la polarisation TM ne voyait que l'indice extraordinaire.

\section{Résultats}

Les bandes interdites obtenues avec le meilleur jeu de paramètres, sont tracées en gris clair sur la figure 50. Elles reproduisent relativement bien à la fois la position spectrale et la largeur des bandes interdites ${ }^{4}$. Les quelques différences qui subsistent concernent principalement les plus petites longueurs $d^{\prime}$ onde $(\lambda$ entre $1,2 \mu \mathrm{m}$ et $1,3 \mu \mathrm{m}$ ) : les bandes interdites calculées se manifestent très peu expérimentalement. Toujours à petite longueur d'onde, le décalage entre expérience et calcul est probablement à mettre sur le compte de la dispersion d'indice. Les paramètres du calcul sont récapitulés dans le tableau 1. Une représentation plus visuelle de la structure modèle, sous forme de carte d'indice, est également donnée sur la figure 51 .

\section{Discussion}

\subsection{Amplitude de la perturbation}

Les mesures montrent sans ambiguïté des largeurs de bandes interdites supérieures en polarisation TM. Dans notre modèle, nous les attribuons à une modulation d'indice de plus grande amplitude pour l'indice extraordinaire $\left(\left|\Delta n_{0}\right|=0,4\right)$ que pour l'indice ordinaire $\left(\left|\Delta n_{0}\right|=0,22\right)$.

4. À noter que les régions grisées correspondent aux fréquences pour lesquelles la constante de propagation prend un partie imaginaire, conduisant à l'atténuation, telle que l'illustre la figure 47. Dans le cas d'une structure finie, la variations de part et d'autre de cette région est peu brutale, en raison des franges d'interférences (qui ne sont pas résolues) dont le contraste décroît progressivement. 

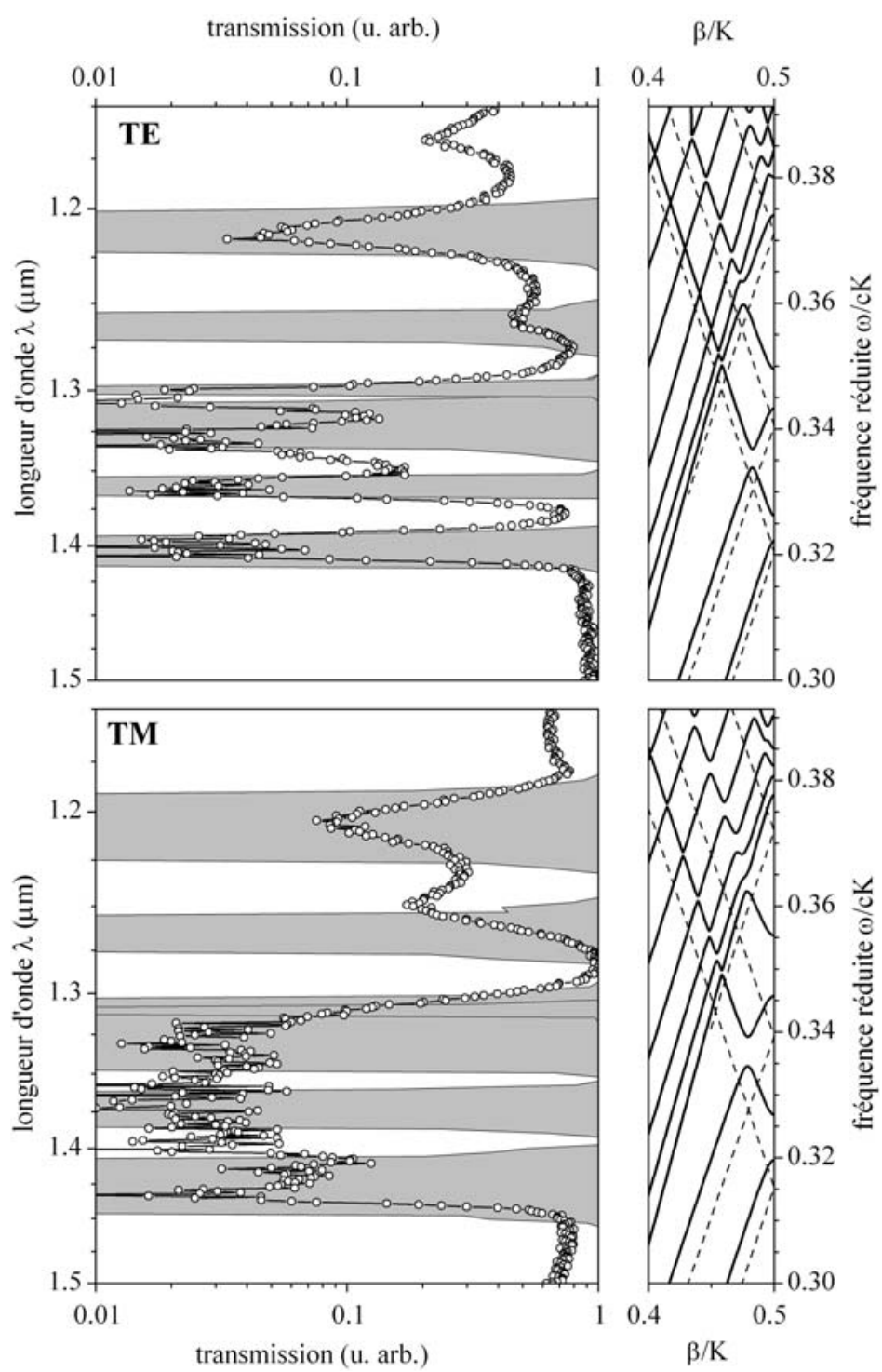

Figure 50. À gauche : comparaison des spectres de transmission avec les bandes interdites calculées par la méthode des modes couplés. À droite : courbes de dispersion obtenues par un calcul rigoureux (méthodes des ondes planes). La région grisée correspond aux continuum de fréquences accessibles par les modes rayonnant à l'extérieur.

[Left: comparison between transmittance spectra and stopbands calculated by means of the coupled modes theory. Right: dispersion curves obtained by a rigorous computation (method of plane waves). The grey region is the continuum of frequencies corresponding to radiating modes.] 
Tableau 1. Valeurs des paramètres utilisés pour le calcul des bandes interdites représentées sur la figure 50. L'épaisseur de la couche guidante est $e_{2}=1,68 \mu \mathrm{m}$. Une représentation graphique de ces paramètres est donnée sur la figure 51.

[Values of the parameters used in the calculation of the stopbands plotted in Figure 50. The thickness of the guiding layer is $e_{2}=1.68 \mu \mathrm{m}$. A graphical view of these parameters is given in Figure 51.]

\begin{tabular}{lcc}
\hline \hline & ordinaire (TE) & extraordinaire (TM) \\
\hline$n_{2}$ (couche guidante) & 1,615 & 1,66 \\
$n_{3}$ (gaine) & 1,31 & 1,32 \\
$\Delta n_{0}$ & $-0,22$ & $-0,4$ \\
$\alpha_{\text {ill }}\left(\mu \mathrm{m}^{-1}\right)$ & 2,0 & 2,0 \\
\hline \hline
\end{tabular}

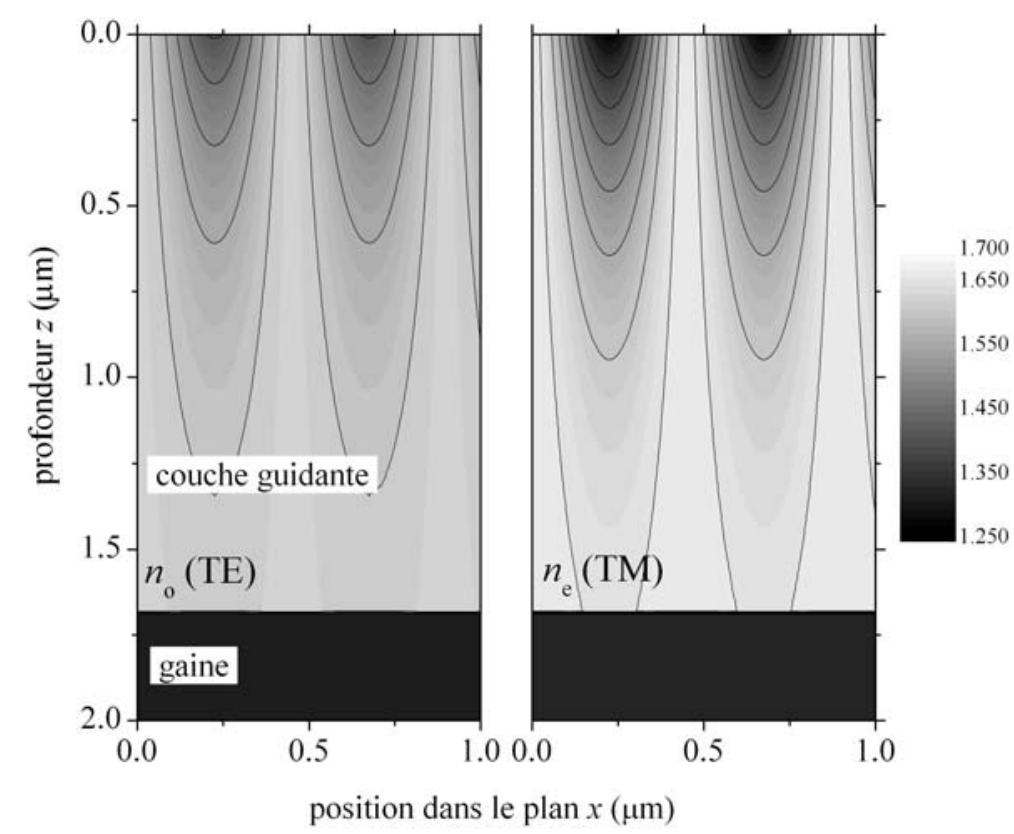

Figure 51. Carte d'indice utilisée pour le calcul des bandes interdites représentées sur la figure 50. L'échelle de niveaux de gris est commune aux deux graphes. Les valeurs exactes des paramètres sont données dans la tableau 1.

[Index map used for the computation of the stopbands plotted in Figure 50. The greyscale is the same for both viewgraphs. The values are given in Table 1.]

Au cours d'études sur la polarisation de la photoluminescence du silicium poreux, Polisski et al. [76] ont proposé un modèle décrivant l'influence d'une illumination polarisée lors de la formation du matériau, en termes d'absorption sélective : partant d'un ensemble de cristallites ellipsoïdales désordonnées, celles dont la plus grande dimension est alignée avec la polarisation de l'illumination absorbent préférentiellement la lumière, ce qui contribue à les arrondir. L'absorption 
de celles qui sont orientées dans le plan perpendiculaire est plus faible, et leur forme est alors affectée dans une moindre mesure. À terme, les zones éclairées sont constituées de cristallites possédant une élongation préférentielle dans le plan $(x z)$, qui contient le champ électrique en polarisation TM. En termes d'indice optique, cette morphologie correspond à un indice plus faible [49] dans ce plan.

Cette interprétation est en accord avec nos résultats. Dans le cas présent, il se trouve que l'effet sur l'indice est colossal, et justifierait une étude plus approfondie de la biréfringence (dans des configurations qui peuvent être nettement plus simples).

La structuration obtenue est encourageante : dans la partie supérieure de la couche, nous avons été capable de générer un contraste d'indice de 0,4 (TM), entre deux régions distantes de $\Lambda / 2=225 \mathrm{~nm}$. Plus généralement, ce résultat montre que le procédé optique n'est manifestement pas limité outre mesure par la diffusion des porteurs, qui étendrait l'influence de la lumière au-delà des régions illuminées. Dans le cas du silicium poreux, ni la durée de vie des porteurs dans la matrice poreuse, ni la distance parcourue avant recombinaison ne sont connues, et nous ne savons pas dans quelle mesure elle intervient, et a fortiori quelle serait la période limite de notre méthode holographique.

Concernant l'atténuation de la perturbation en profondeur, il faut bien avouer que la valeur de $\alpha_{\text {ill }}=2 \mu \mathrm{m}^{-1}$, commune aux deux polarisations, est quelque peu décevante : le coefficient d'absorption du silicium poreux à $\lambda=0,5 \mu \mathrm{m}$ est

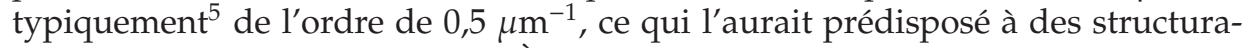
tions nettement plus profondes. À ce stade, aucun argument optique ne permet d'expliquer une atténuation supplémentaire, les réponses sont probablement à rechercher du côté de comportement dynamique des porteurs photogénérés au cœur du matériau. Quoi qu'il en soit, cela constitue une limite majeure à notre procédé de structuration.

\subsection{Conséquences de l'asymétrie de la perturbation}

Si nous revenons sur l'expression du coefficient de couplage, en polarisation TE par exemple,

$$
\kappa_{1, l m}^{(\mathrm{TE})} \propto \int_{-\infty}^{+\infty} \Delta n^{2}(z) E_{y}^{(l)}(z) E_{y}^{(m)}(z) \mathrm{d} z,
$$

nous pouvons faire quelques remarques quant à la sélectivité liée à la symétrie de la perturbation. En particulier, nous voyons que si cette dernière est homogène en profondeur, nous avons, en raison des propriétés d'orthogonalité des modes guidés, $\kappa_{1, l m}=0$ dès que $l \neq m$ : dit autrement, seuls les couplages diagonaux sont possibles.

Nous sommes manifestement dans le cas contraire, car certaines bandes interdites (cf. Fig. 50, notamment pour $\lambda \simeq 1,35 \mu \mathrm{m}$, dans les deux polarisations)

5. Cette valeur typique ressort des nombreux spectres de réflectivité de couches simples que nous avons eu l'occasion de mesurer. Par exemple, sur la figure 15, page 32, à $\lambda=0,5 \mu \mathrm{m}, \operatorname{Im}(n) \simeq 0,02$ soit $\alpha \simeq 0,5 \mu \mathrm{m}^{-1}$. 
Tableau 2. Modules des coefficients de couplage $\kappa_{1, l m}\left(\mathrm{en} \mu \mathrm{m}^{-1}\right)$ obtenus pour les différents couples $(l, m)$ pour chaque polarisation. Les valeurs manquantes sont obtenues par symétrie car $\kappa_{1, l m}=\kappa_{1, m l}$. Au centre de chaque bande interdite, la dépendance du champ selon $x$ est de la forme $\exp ( \pm \kappa x)$.

[Modulus of the coupling constants $\kappa_{1, l m}$ (in $\mu \mathrm{m}^{-1}$ ) obtained for different values of $(l, m)$. The missing values can be obtained by symmetry using $\kappa_{1, l m}=\kappa_{1, m l}$. In the middle of each stopband, the field decreases in $x$ following $\exp ( \pm \kappa x)$.]

\begin{tabular}{ccccccccc}
\hline \hline TE & 0 & 1 & 2 & & TM & 0 & 1 & 2 \\
\hline 0 & 0,059 & 0,046 & 0,012 & & 0 & 0,096 & 0,073 & - \\
1 & & 0,073 & 0,047 & & 1 & & 0,116 & - \\
2 & & & 0,073 & & 2 & & & 0,075 \\
\hline \hline
\end{tabular}

ne peuvent être interprétées qu'en termes de couplage non diagonal. Notre perturbation est certes dissymétrique, par nature puisqu'elle a une action limitée à $z \geq 0$, mais cette dissymétrie n'est pas suffisante pour interpréter les largeurs de bandes mesurées, et c'est au moyen de telles considérations que le coefficient d'atténuation $\alpha_{\text {ill }}$ a pu être évalué, indépendamment de $\Delta n_{0}$. Les coefficients de couplage $\kappa_{1, l m}$ obtenus sont répertoriés pour tous les couples $(l, m)$ dans le tableau 2. Les couplages non diagonaux du type $(l, l \pm 1)$ apparaissent donc avec une efficacité à peine inférieure à celle des couplages diagonaux.

Plus généralement, ces résultats insistent sur le rôle sélectif joué par la symétrie de la perturbation. Récemment, Olivier et al. ont rapporté des observations similaires sur le guidage que l'on obtient en supprimant un certain nombre de rangées de trous dans un cristal photonique bidimensionnel : si les rangées de trous de part et d'autre du canal sont en parfait vis-à-vis, la périodicité du cristal photonique environnant peut donner lieu à des « minibandes interdites » [99] selon la direction de propagation longitudinale, manifestation de couplages diagonaux 6 .

\subsection{Validité de la méthode perturbative}

Lorsqu'on utilise une méthode perturbative, il est prudent de s'assurer qu'elle repose sur une approximation raisonnable. Dans la mesure où nous avions tout à apprendre de la structure étudiée, cette vérification est effectuée a posteriori.

La principale approximation concerne les modes de la structure perturbée, dont on suppose qu'ils sont proches de ce qu'ils seraient en l'absence de perturbation. Avec la carte d'indice que nous proposons (Fig. 51), l'indice moyen de la couche guidante est $\bar{n}_{2}^{\mathrm{TE}}=1,59$ (contre $n_{2}=1,615$ pour la couche non perturbée) pour la polarisation TE, et $\bar{n}_{2}^{\mathrm{TM}}=1,61$ (contre $n_{2}=1,66$ ) pour la polarisation TM. L'écart est faible, mais pas négligeable (à plus forte raison en polarisation TM).

Une preuve expérimentale de la variation relativement faible de l'indice moyen de la couche guidante peut être apportée par des mesures à angle variable.

6. On peut palier cet effet en décalant d'une demi-période les rangées en vis-à-vis : les couplages entre modes de même parité (a fortiori les couplages diagonaux) sont alors interdits, mais les couplages non-diagonaux entre modes de parité opposée sont possibles. 
Nous avons réalisé différentes mesures de transmission en pivotant l'échantillon dans son plan, jusqu'à un azimut de $30^{\circ}$. La série de spectres obtenue est tracée sur la figure 52. Dans la mesure où la constante de propagation selon $x$ devient $\beta \cos \phi_{\text {guid, }}$ où $\phi_{\text {guid }}$ est l'azimut dans la couche guidante déterminé par l'indice $n_{2}$, les bandes d'arrêt sont obtenues pour une fréquence $\omega / \cos \phi_{\text {guid }}$. La dépendance angulaire de la fréquence de la bande la plus marquée a été pointée pour les différents angles (croix sur la Fig. 52), et nous avons reporté sur la même figure le calcul de cette dépendance, en donnant à la couche guidante l'indice non-perturbé. Bien que nous ne puissions prétendre mesurer précisément l'indice moyen, nous pouvons cependant constater que la courbe expérimental s'écarte peu de la dépendance théorique d'un indice non-perturbé ${ }^{7}$.

Pour avoir une idée plus précise de l'erreur commise, nous avons confronté nos bandes interdites à un calcul rigoureux, au moyen la méthode des ondes planes de Johnson et Joannopoulos [95]. Le diagramme de dispersion, résultat du calcul mené sur la même distribution d'indice, est reporté dans la partie droite de la figure 50, page 98. La coïncidence des bandes interdites calculées par les deux méthodes est tout à fait satisfaisante. Les différences que l'on peut constater (fréquence ou largeur) sont trop faibles pour remettre en cause la validité de la méthode perturbative. Dans nos conditions, cette dernière est sans conteste la plus avantageuse, dans la mesure où son extrême rapidité d'exécution s'accommode sans problème d'une procédure d'ajustement de paramètres.

\subsection{Sélectivité du couplage par la tranche}

Nous avons vu que les anticroisements des courbes de dispersion se manifestaient expérimentalement par une chute de l'intensité transmise par le guide. Pourtant, bien souvent, il apparaît sur les diagrammes de dispersion que d'autres modes, non-concernés par le couplage, seraient susceptibles de se propager efficacement. Il n'en est rien : sur la figure 50 (indépendamment de la polarisation), le premier anticroisement à basse fréquence est la conséquence du couplage $(0,0)$; rien ne s'oppose à la propagation du mode $l=1$, mais ce n'est manifestement pas le cas, puisque l'intensité transmise chute brutalement. Le mode $l=1$ est pourtant bien transporté dans le guide car le couplage $(1,1)$ produit de nouveau une chute d'intensité (à plus haute fréquence). Il semble donc que l'opposition que rencontre un mode pour se propager se communique même aux modes non-concernés.

Les modèles que nous avons utilisés jusqu'à présent considéraient soit un guide infini dont une portion finie est perturbée (méthode des modes couplés), soit un guide périodique infini (méthode des ondes planes). Notre cas expérimental se distingue de ces deux approches dans la mesure où la lumière est injectée directement par la tranche du guide périodique : par rapport à la méthode des modes couplés, les modes du guide non-perturbé n'ont pas le temps de se construire en amont de la perturbation; par rapport à la méthode des ondes

7. À noter que cette série de mesures constitue une preuve irréfutable — si nous n'en étions pas convaincu - que les chutes d'intensité sont dues à un effet de bande interdite, balayant toute interprétation en termes d'absorption ou de diffusion. 


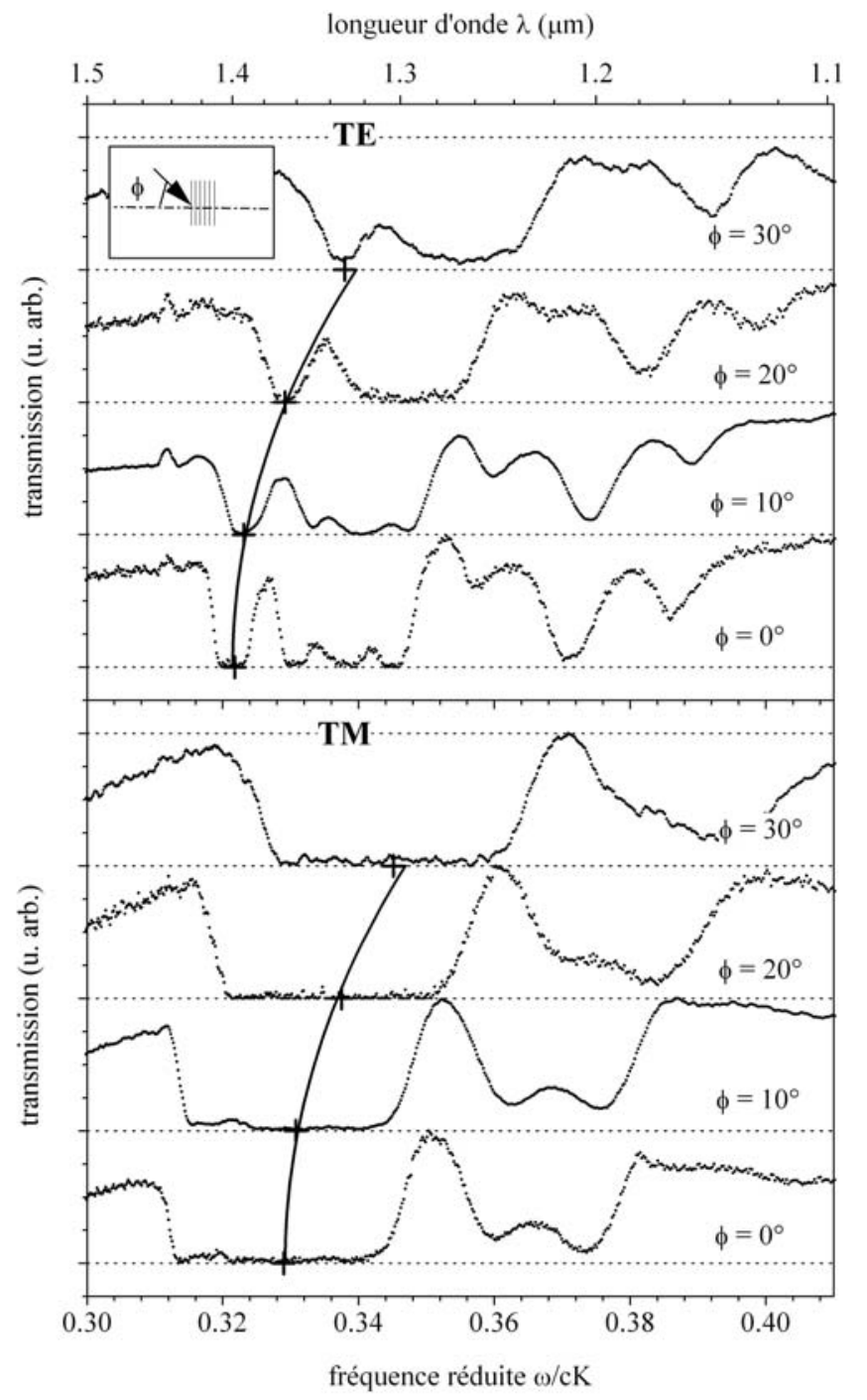

Figure 52. Évolution des spectres de transmission pour différents azimuts $\phi$ (définis dans l'air). Les différents spectres, tracés en échelle linéaire, ont été décalés verticalement pour une meilleure lisibilité. Les croix montrent la dépendance spectrale d'une certaine bande interdite. La ligne en trait plein correspond à la dépendance calculée par un modèle d'indice moyen. La signification de l'azimut $\phi$ est rappelée dans l'insert.

[Blue shift of the transmittance spectra when increasing the angle $\phi$ (given in air). The different spectra, plotted in linear scale, are shifted vertically to be more legible. The crosses show the spectral shift of an arbitrary stopband. The solid line corresponds to the shift calculated by a model of mean index. The definition of $\phi$ is reminded in the inset.] 
planes, le couplage par la tranche nécessite une intervention drastique des effets de bord.

Ainsi tout laisse à penser que les effets rapportés sont dus au couplage par la tranche. Nous avons insisté en $\S 3.5$., chapitre 2 sur le rôle sélectif joué par la distribution de champ électrique au niveau de la tranche sur l'efficacité d'injection dans les différents modes du guide. Dans ces conditions, il est tout à fait concevable que la réflexion d'un certain mode bouleverse le champ sur la tranche, et donc les conditions de couplage des autres modes. Plus récemment, nous avons confronté ces résultats avec la méthode de Pendry et al., mieux adaptée à notre système, car elle est basée sur une technique de matrices de transfert (à trois dimensions) [100,101], permettant de travailler sur des structures finies et d'avoir accès aux coefficients de transmission. Le calcul mené sur une structure proche de la nôtre corrobore nos mesures, comme le montre la figure 53 : chaque couplage se traduit par une chute du coefficient de transmission, indépendamment du fait que d'autres modes pourraient se propager. Il serait intéressant d'étudier si l'effet persiste lorsque la perturbation intervient à une distance respectable de la tranche d'entrée du guide.

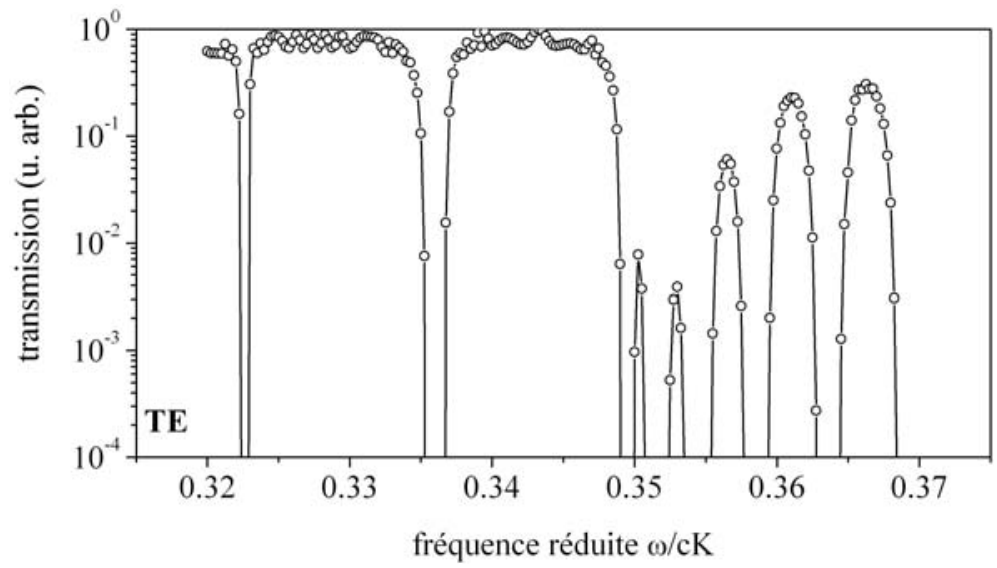

Figure 53. Transmission d'un guide périodique fini calculée par la méthode des matrices de transfert. Les chutes d'intensité correspondent à une bande interdite. Comme dans nos mesures, les autres modes qui pourraient se propager librement ne sont pas couplés dans le guide. La structure calculée est similaire à celle de la figure 50, mais le calcul porte ici sur 65000 périodes.

[Transmittance of a periodic waveguide of finite length calculated by a transfer matrix method. Each decrease of intensity corresponds to a stopband. Like in our measurements, no light is injected into other modes, even if these are allowed to propagate in the waveguide. The strucure is the same as those of Figure 50, but the computation is performed on 65,000 periods.] 


\subsection{Intérêt pour l'émission de surface}

Une bande interdite pour la propagation dans le plan est susceptible d'interdire l'émission dans la direction considérée. Cependant, dans le cas du silicium poreux, l'efficacité quantique de la luminescence est très faible, si bien que toute répercussion sur l'émission verticale est vaine. Par contre, une redirection de l'émission planaire est envisageable. En effet, la perturbation est susceptible dans certaines conditions de coupler les modes guidés avec le continuum des modes rayonnants. Ceci est particulièrement visible sur la figure 46 , page 88 , lorsque les modes du guide se trouvent repliés par la périodicité dans le cône de lumière, représenté en grisé. Pour cette raison, les réseaux ont été utilisés très tôt comme un moyen de coupler un faisceau extérieur dans un guide d'onde [102,103], s'affranchissant ainsi d'un couplage par la tranche souvent fastidieux.

Beaucoup plus récemment, une utilisation «inverse » de ce phénomène a été proposée, pour augmenter l'émission verticale de structures planaires [104], dont une part non-négligeable de l'intensité est émise — en pure perte — dans des modes guidés.

Il se trouve que le guide périodique que nous étudions dans ce chapitre est luminescent ${ }^{8}$. En outre, la gamme spectrale de la luminescence $(0,6-0,9 \mu \mathrm{m})$ correspond sur les diagrammes de dispersion à des fréquences pour lesquelles ce type de couplage est possible. Nous avons donc essayé de vérifier si l'efficacité d'émission se trouvait changée par la présence de la structuration périodique. Une étude comparative de deux échantillons (un avec et un sans structuration) étant impossible, dans la mesure où le procédé de structuration holographique modifie l'ensemble de la structure (indices, absorptions, luminescence, etc.), nous avons opté pour une caractérisation angulaire de l'échantillon structuré.

En effet, si nous considérons les vecteurs d'ondes associés aux modes d'un guide non-perturbé à une fréquence donnée, ils décrivent des cercles concentriques dans le plan $\left(\mathrm{O}, k_{x}, k_{y}\right)$, comme l'illustre la figure 54 . Le cône de lumière, grisé sur la figure, apparaît sous la forme d'un disque centré, de rayon $\omega / c$. En présence de la perturbation, périodique selon $x$, ces modes sont reportés périodiquement selon $k_{x}$ de quantités multiples de $K$, et des modes sont susceptibles de pénétrer dans le cône de lumière.

Dans ces conditions, l'effet de la période doit se manifester si l'on mesure l'émission dans le cône de lumière, en se déplaçant parcourant l'axe $k_{x}$, puis l'axe $k_{y}$. Ce peut être fait facilement sur le banc expérimental détaillé en $\S 2.2$., chapitre 4, en mesurant la photoluminescence émise par la surface en changeant l'angle de mesure $\theta$ selon la direction $x$ (nous appellerons l'angle $\theta_{x}$ ), puis selon $y\left(\theta_{y}\right)$, après avoir simplement tourné l'échantillon de $90^{\circ}$ sur son support.

Le résultat des mesures, effectuées entre $0^{\circ}$ et $70^{\circ}$, est tracé sur la figure 55. Pour chaque polarisation, nous constatons que la série de spectres obtenus en faisant varier $\theta_{x}$ ne présente pas de différence notable par rapport à celle obtenue

8. L'émission provient probablement des régions de très forte porosité qui forment le faible indice de la perturbation périodique. 


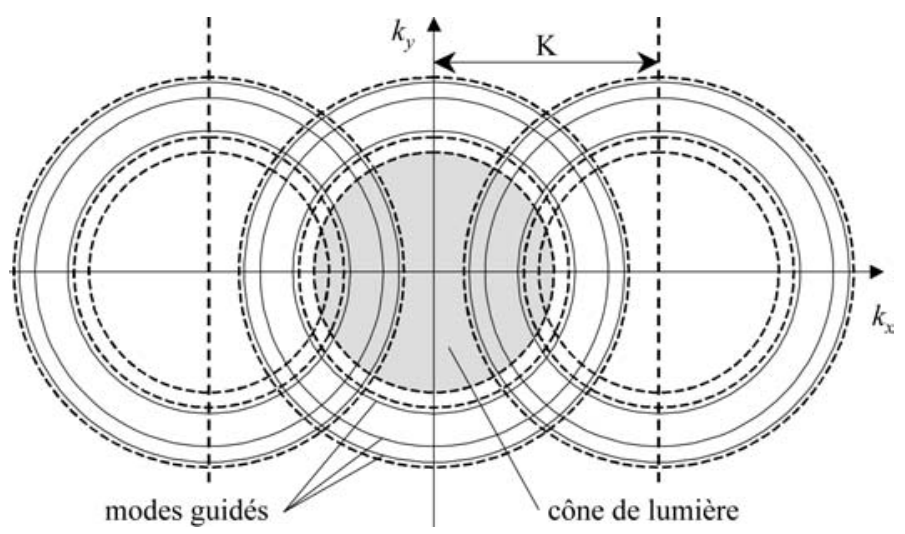

Figure 54. Représentation schématique des modes (trait épais) du guide périodique dans le plan $\left(\mathrm{O}, k_{x}, k_{y}\right)$, à une fréquence donnée. Le disque grisé est le cône de lumière. Les modes guidés qui y pénètrent sont susceptibles d'être couplés avec le continuum des modes rayonnants.

[Schematic view in the plane $\left(\mathrm{O}, k_{x}, k_{y}\right)$ of the modes (thin solid lines) of the periodic waveguide, at a given frequency. The grey disc is the light cone. Guided modes within this disc mean that they can be coupled to the continuum of radiated modes.]

en jouant sur $\theta_{y}$. L'échantillon étudié ne bénéficie donc d'aucune amélioration de l'intensité émise.

Aurions-nous pu prévoir ce résultat? Sans doute. Connaissant la structuration d'indice, des méthodes numériques (certes relativement complexes) [104] permettent de calculer la modification d'émission due au couplage des modes guidés vers l'extérieur ${ }^{9}$. Mais plus raisonnablement, il semble que ce soit l'absorption, importante dans cette gamme spectrale, qui limite la distance sondée par la lumière émise, et donc l'efficacité de diffraction par la structure périodique. L'ordre de grandeur de l'absorption peut être donné par les mesures réalisées en $\S 3.1$., chapitre 6. Les pertes mesurées étaient de l'ordre de plusieurs dizaines de $\mathrm{cm}^{-1}$, soit une atténuation d'un facteur $e$ au bout d'une fraction de millimètre. Cette distance est manifestement trop faible pour l'efficacité de notre réseau.

Dans ce dernier chapitre, nous nous sommes intéressé à une structure planaire guidante, à laquelle nous avons ajouté une modulation d'indice dans la direction longitudinale. Au moyen de mesures de transmission d'intensité guidée en lumière blanche, nous avons mis en évidence de nombreuses bandes interdites, associées aux multiples couplages entre les différents modes, caractérisées par des chutes d'intensité qui atteignent deux ordres de grandeurs.

L'utilisation d'une méthode perturbative nous a permis de déterminer quantitativement l'amplitude et la profondeur effective de pénétration de la modulation d'indice. Les résultats obtenus ont été discutés en termes d'efficacité de couplage.

9. La méthode des modes couplés, sous sa forme présente, ne permet pas de calculer les efficacités associées à ces couplages, car nous avons ignoré les modes rayonnants dans l'établissement de l'expression des équations de couplage. 


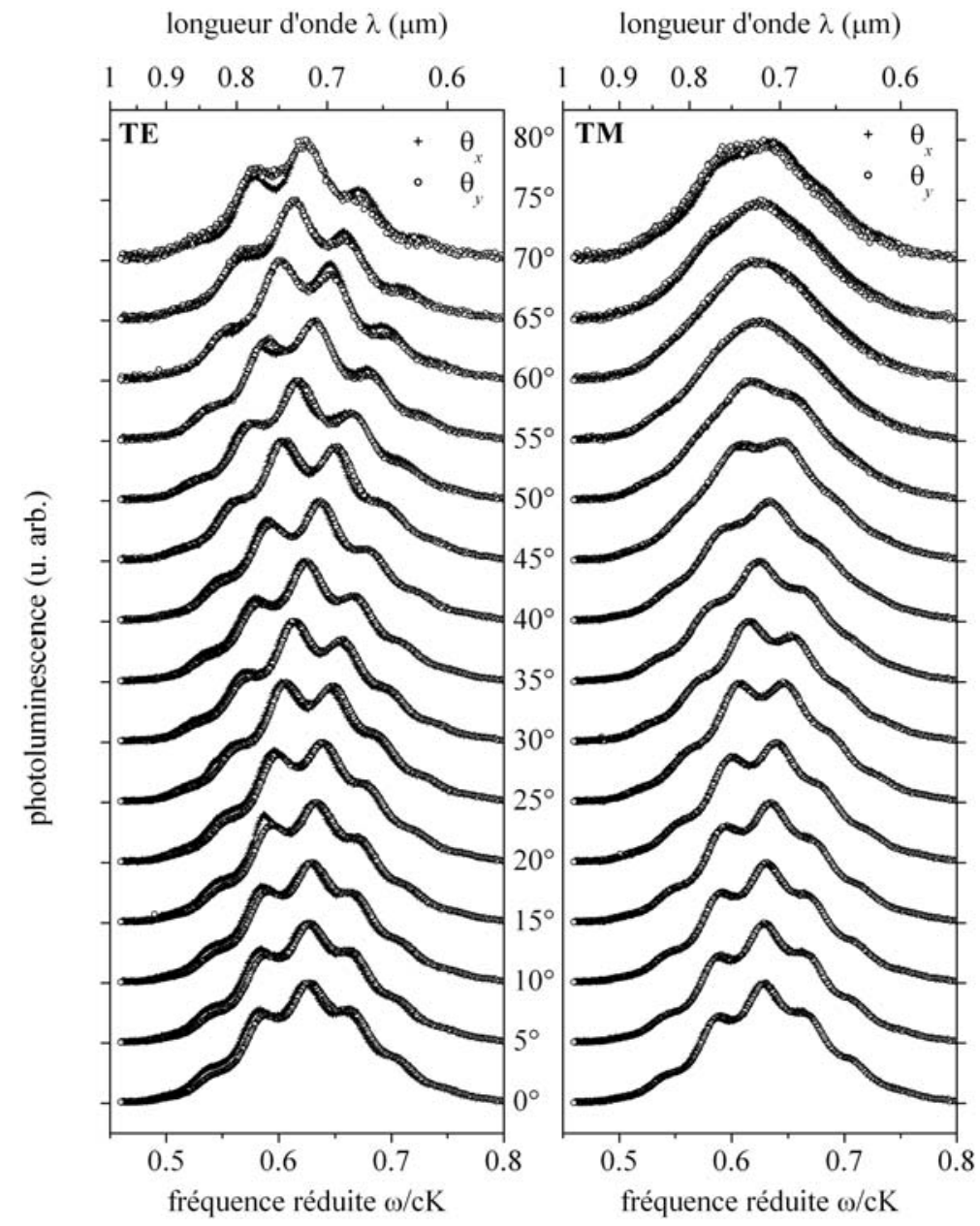

Figure 55. Étude angulaire de l'émission d'une structure guidante périodique. Pour chaque polarisation, les mesures ont été réalisées en collectant la lumière émise par la surface sous différents angles $\theta$, dans la direction de $x\left(\theta_{x}\right.$, tracés en croix $\left.(+)\right)$ et dans la direction $y\left(\theta_{y}\right.$, tracés en cercles (०)) de 0 à $70^{\circ}$. Les spectres des différents angles sont décalés verticalement pour une meilleure lisibilité, l'angle de mesure étant indiqué en face de chaque spectre.

[Angular study of the vertical emission of a periodic guiding structure. For each polarization, measurements have been performed by collecting the emitted light at different angles $\theta$, in the $x$-direction $\left(\theta_{x}\right.$, plotted in crosses $\left.(+)\right)$ and in the $y$-direction $\left(\theta_{y}\right.$, plotted in circles (o)) from 0 to $70^{\circ}$. Spectra of different angles have been shifted vertically to be more legible. The angle is indicated close to each spectrum.] 
Après nous être assuré de la validité de l'approche perturbative, nous avons insisté sur le rôle sélectif joué par la symétrie de la perturbation, et nous avons mis en évidence un phénomène de couplage sélectif, lors du couplage par la tranche du guide périodique. Sur l'échantillon présent, nous avons constaté que la nature périodique n'apportait aucun bénéfice en terme d'émission verticale, probablement à cause d'une absorption trop grande dans la gamme étudiée. 
Dans cet ouvrage, nous nous sommes intéressé au contrôle de la lumière dans des microstructures planaires de silicium nanoporeux.

L'étude a commencé par une caractérisation de l'émission intrinsèque du matériau, d'un point de vue spectral et angulaire. Nous avons constaté qu'elle était grosso modo isotrope. Nous avons également montré que les différences d'intensité et d'angle d'émission qui distinguent les polarisations d'émission par la surface ne sont qu'une conséquence de la réponse passive de la structure optique, dans laquelle un rôle majeur est joué par l'absorption. Dans ce contexte, la modélisation de l'émission prenant en compte ce phénomène est apparue comme un outil déterminant.

Nous avons ensuite cherché à favoriser la propagation latérale, au moyen d'une structuration verticale de l'indice. Nous avons réalisé deux types de structures guidantes, exploitant soit un guidage conventionnel par réflexion totale interne, soit un guidage par réflexion de Bragg sur un empilement périodique. Les études qui ont été menées, que ce soit en analysant la transmission de lumière blanche ou l'émission par la tranche de la structure, nous ont convaincu d'un guidage efficace dans le proche infrarouge, les pertes s'élevant dans le cas le plus favorable à quelques $\mathrm{cm}^{-1}$. Nous avons mis en évidence le rôle joué principalement par la diffusion de volume (diffusion Rayleigh) aux longueurs d'onde où elle n'est pas masquée par l'absorption.

Nous nous sommes ensuite intéressé au contrôle de la propagation guidée par un effet de bande interdite photonique, obtenu par une structuration périodique de l'indice le long de la direction de propagation. La transmission mesurée en lumière blanche sur un guide multimode, structuré par un procédé holographique, nous a révélé la présence de multiples bandes interdites. Nous avons interprété ces multiples bandes en termes des différents couplages entre modes, couplages diagonaux et non diagonaux, ces derniers étant rendus possibles par la diminution en profondeur de l'efficacité de la perturbation périodique. La confrontation des mesures avec une modélisation par la méthode des modes couplés nous a permis d'établir une carte de l'indice de la structure. À cette occasion, nous avons mis en évidence une biréfringence marquée dans les régions illuminées lors du procédé holographique, aboutissant à une diminution d'indice deux fois plus importante pour l'indice extraordinaire $(\Delta n=-0,4$ en surface) que pour l'indice ordinaire $(\Delta n=-0,22)$. Ce contraste, obtenu sur une distance de $225 \mathrm{~nm}$ 
est encourageant. Le constat est plus mitigé concernant la profondeur effective de la perturbation, de l'ordre de $0,5 \mu \mathrm{m}$, valeur nettement inférieure à ce que l'absorption du matériau pouvait laisser espérer. Dans ces conditions, les valeurs des coefficients de couplage que nous avons estimés indiquent une atténuation $\mathrm{du}$ faisceau incident au bout d'une propagation effective de l'ordre de quelques dizaines de microns. Nous avons également mis en évidence la sélectivité du couplage par la tranche, lorsque la tranche d'injection se prolonge immédiatement par un guide périodique. Nous avons constaté que la réflexion d'un mode pouvait empêcher l'injection de lumière, même dans des modes susceptibles de se propager librement.

À ce stade, nous sommes loin d'avoir identifié les mécanismes qui entrent en jeu lors de la photodissolution; une étude à différentes périodes et longueurs d'onde pourrait probablement fournir quelques pistes, quant à l'atténuation de la perturbation en profondeur à l'évaluation de la période limite et de l'évolution de la profondeur de structuration. Des évaluations quantitatives de la réflectivité, de l'atténuation dans et entre les bandes interdites seraient également intéressantes. La sélectivité du couplage par la tranche mérite également quelques éclaircissements, notamment par une étude des structures pour lesquelles la perturbation intervient suffisamment loin de la tranche de couplage.

Concernant la structuration bi- ou tri-directionnelle dans le plan, nous en avons déjà testé — avec succès — la faisabilité à période plus importante. À faible période, il ne s'agit a priori que d'une formalité, la multiplication des faisceaux d'illumination ne devant pas apporter de difficulté autre que matérielle. Si nous nous sommes limité dans le cadre de cette étude à une structuration unidirectionnelle, c'était davantage pour simplifier le problème d'un point de vue théorique.

Quoi qu'il en soit, le silicium nanoporeux mérite de figurer dans la liste des matériaux pour l'optique. S'il ne peut rivaliser avec le fort indice des semiconducteurs massifs, les valeurs d'indices accessibles apparentent le silicium poreux à la plupart des matériaux diélectriques (dans le proche infrarouge), avec un net avantage en termes de facilité de structuration verticale.

Concernant la structuration latérale, les résultats que nous avons obtenus indiquent un contraste d'indice et surtout une profondeur de structuration handicapant pour la réalisation de structures planaires à bande interdite photonique (circuits optiques intégrés, etc.). En optique intégrée conventionnelle, le silicium nanoporeux manque d'arguments pour détrôner les matériaux actuels (verres dopés). En termes d'émission verticale du silicium poreux, nous n'avons pas mis en évidence d'effet notable, les contrastes d'indice nécessitant une longueur de propagation que l'absorption ne permet pas.

Cependant, les études menées dans notre groupe sur le silicium poreux de type $n^{+}$, nettement plus marginal dans la communauté du silicium poreux, et pour lequel l'anodisation et la structuration se font simultanément, semblent indiquer des aptitudes particulières quant à la profondeur de structuration lumineuse. Cette voie mériterait d'être poursuivie. C'est également le cas des recherches entreprises par certains groupes quant à l'introduction d'erbium [105], émetteur 
efficace dans la gamme de transparence du silicium poreux, et désormais omniprésent dans les réseaux de télécommunication.

Ces résultats ne doivent pas occulter l'extrême complexité du matériau, qui laisse encore de nombreuse question ouvertes. Cet aspect qui excite tant les chercheurs des sciences fondamentales est cependant aujourd'hui une sévère contrainte en termes d'applications, et rien ne permet aujourd'hui de prédire si le silicium poreux aura une autre destinée que celle de curiosité de laboratoire.

À moins que sa place ne soit dans les salles de travaux pratiques des universités. Lors de la deuxième conférence internationale Porous Semiconductors Science and Technology qui s'est tenue en mars 2000 à Madrid, Parkhutik et Canham [106] ont rapporté un programme mené en ce sens pendant plusieurs années avec succès à l'université de technologie de Valence (Espagne). L'expérience mériterait d'être étendue. La simplicité, la rapidité et le faible coût de fabrication permettrait d'associer élaboration et caractérisation. L'éventail des propriétés (électriques, optiques, mécaniques, structurales, chimiques, etc.) pourraient le mettre au service de l'enseignement de nombreux domaines de la physique et des sciences des matériaux, et ce sans faire abstraction - et c'est le plus important, lorsqu'on forme de futurs chercheurs - du caractère éminemment complexe et mal compris du matériau. Peut-on rêver d'une plus noble destinée?

\section{Remerciements}

Cet ouvrage est très largement inspiré de ma thèse de doctorat effectuée au sein du Laboratoire de Spectrométrie Physique (UMR 5588 du CNRS) de l'université Joseph Fourier Grenoble 1. Je tiens à exprimer ma reconnaissance à Robert Romestain, mon directeur de thèse, pour l'encadrement scienfitique dont il m'a fait bénéficier et la confiance qu'il m'a accordée. Merci également à tous les membres du laboratoire et tout particulièrement à Susanna Setzu, Jean-Claude Vial et Davide Loi pour leur soutien et leurs précieux conseils.

Ce travail a été financé par une allocation de recherche du ministère de d'Éducation nationale, et a bénéficié du soutien financier du Centre national de la recherche scientifique dans le cadre du programme Ultimatech. 


\section{Annexes}

\section{Annexe A : Expression du vecteur de Poynting}

Nous donnons dans cette annexe quelques étapes du calcul qui permet d'établir l'expression du vecteur de Poynting dans le formalisme de matrices de transfert, tel qu'il intervient en $\S 2$., chapitre 6 .

\section{A.1. Polarisation TE}

En polarisation TE, le champ électrique est parallèle à $\hat{y}$, il s'écrit donc dans chaque couche $i$ sous la forme

$$
\mathbf{E}_{i}=\left[\begin{array}{l}
0 \\
E_{i \uparrow} \mathrm{e}^{\mathrm{i}\left(\beta x+k_{z, i} z-\omega t\right)}+E_{i \downarrow} \mathrm{e}^{\mathrm{i}\left(\beta x-k_{z, i} z-\omega t\right)} \\
0
\end{array}\right],
$$

où $\beta$ est réel (invariance dans le plan) mais $k_{z, i}$ peut être complexe. La première équation de Maxwell (cf. Éqs. (2.1)) nous permet d'en déduire le champ magnétique $\mathbf{H}_{i}$ comme

$$
\mathbf{H}_{i}=\frac{1}{\omega \mu_{0}}\left[\begin{array}{l}
k_{z, i}\left\{-E_{i \uparrow} \mathrm{e}^{\mathrm{i}\left(\beta x+k_{z, i} z-\omega t\right)}+E_{i \downarrow} \mathrm{e}^{\mathrm{i}\left(\beta x-k_{z, i} z-\omega t\right)}\right\} \\
\beta\left\{E_{i \uparrow} \mathrm{e}^{\mathrm{i}\left(\beta x+k_{z, i} z-\omega t\right)}+E_{i \downarrow} \mathrm{e}^{\mathrm{i}\left(\beta x-k_{z, i} z-\omega t\right)}\right\}
\end{array}\right] .
$$

On a donc

$$
\mathbf{E}_{i} \wedge \mathbf{H}_{i}^{*}=\frac{1}{\omega \mu_{0}}\left[\begin{array}{l}
\beta\left\{\left|E_{i \uparrow}\right|^{2} \mathrm{e}^{-2 \operatorname{Im}\left(k_{z, i}\right) z}+\left|E_{i \downarrow}\right|^{2} \mathrm{e}^{+2 \operatorname{Im}\left(k_{z, i}\right) z}+2 \operatorname{Re}\left[E_{i \uparrow} E_{i \downarrow}^{*} \mathrm{e}^{+2 i \operatorname{Re}\left(k_{z, i}\right) z}\right]\right\} \\
k_{z, i}^{*}\left\{\left|E_{i \uparrow}\right|^{2} \mathrm{e}^{-2 \operatorname{Im}\left(k_{z, i}\right) z}-\left|E_{i \downarrow}\right|^{2} \mathrm{e}^{+2 \operatorname{Im}\left(k_{z, i}\right) z}-2 \operatorname{iIm}\left[E_{i \uparrow} E_{i \downarrow}^{*} \mathrm{e}^{+2 i \operatorname{Re}\left(k_{z, i}\right) z}\right]\right\}
\end{array}\right],
$$


ce qui permet d'écrire le vecteur de Poynting, d'après l'équation (2.5),

$$
\mathbf{P}(\beta, z)=\frac{1}{2 \omega \mu_{0}}\left[\begin{array}{l}
\beta\left\{\left|E_{i \uparrow}\right|^{2} \mathrm{e}^{-2 \operatorname{Im}\left(k_{z, i}\right) z}+\left|E_{i \downarrow}\right|^{2} \mathrm{e}^{+2 \operatorname{Im}\left(k_{z, i}\right) z}+2 \operatorname{Re}\left[E_{i \uparrow} E_{i \downarrow}^{*} \mathrm{e}^{+2 i \operatorname{Re}\left(k_{z, i}\right) z}\right]\right\} \\
\operatorname{Re}\left(k_{z, i}\right)\left\{\left|E_{i \uparrow}\right|^{2} \mathrm{e}^{-2 \operatorname{Im}\left(k_{z, i}\right) z}-\left|E_{i \downarrow}\right|^{2} \mathrm{e}^{+2 \operatorname{Im}\left(k_{z, i}\right) z}\right\} \\
-2 \operatorname{Im}\left(k_{z, i}\right) \operatorname{Im}\left[E_{i \uparrow} E_{i \downarrow}^{*} \mathrm{e} d^{+2 i \operatorname{Re}\left(k_{z, i}\right) z}\right]
\end{array}\right] .
$$

Remarque. Dans le cas où $k_{z, i}$ est imaginaire pur (ondes évanescentes), le dernier terme de la composante $P_{z}(\beta, z)$ montre que la superposition des deux ondes rend possible un flux d'énergie perpendiculairement au plan.

\section{A.2. Polarisation TM}

En polarisation TM, il est judicieux de partir de l'expression du champ magnétique. La démarche est similaire :

$$
\mathbf{H}_{i}=\left[\begin{array}{l}
0 \\
n_{i}\left\{E_{i \uparrow} \mathrm{e}^{\mathrm{i}\left(\beta x+k_{z, i} z-\omega t\right)}-E_{i \downarrow} \mathrm{e}^{\mathrm{i}\left(\beta x-k_{z, i} z-\omega t\right)}\right\} \\
0
\end{array}\right],
$$

donne, grâce à l'expression de $\nabla \wedge \mathbf{H}$ donnée dans les équations (2.1),

$$
\mathbf{E}_{i}=\frac{1}{\omega \epsilon_{0} n_{i}}\left[\begin{array}{l}
k_{z, i}\left\{E_{i \uparrow} \mathrm{e}^{\mathrm{i}\left(\beta x+k_{z, i} z-\omega t\right)}+E_{i \downarrow} \mathrm{e}^{\mathrm{i}\left(\beta x-k_{z, i} z-\omega t\right)}\right\} \\
\beta\left\{-E_{i \uparrow} \mathrm{e}^{\mathrm{i}\left(\beta x+k_{z, i} z-\omega t\right)}+E_{i \downarrow} \mathrm{e}^{\mathrm{i}\left(\beta x-k_{z, i} z-\omega t\right)}\right\}
\end{array}\right],
$$

d'où

$$
\mathbf{E}_{i} \wedge \mathbf{H}_{i}^{*}=\frac{n_{i}^{*}}{\omega \epsilon_{0} n_{i}}\left[\begin{array}{l}
\beta\left\{\left|E_{i \uparrow}\right|^{2} \mathrm{e}^{-2 \operatorname{Im}\left(k_{z, i}\right) z}+\left|E_{i \downarrow}\right|^{2} \mathrm{e}^{+2 \operatorname{Im}\left(k_{z, i}\right) z}-2 \operatorname{Re}\left[E_{i \uparrow} E_{i \downarrow}^{*} \mathrm{e}^{+2 \operatorname{Re}\left(k_{z, i}\right) z}\right]\right\} \\
k_{z, i}\left\{\left|E_{i \uparrow}\right|^{2} \mathrm{e}^{-2 \operatorname{Im}\left(k_{z, i}\right) z}-\left|E_{i \downarrow}\right|^{2} \mathrm{e}^{+2 \operatorname{Im}\left(k_{z, i}\right) z}-2 \operatorname{iIm}\left[E_{i \uparrow} E_{i \downarrow}^{*} \mathrm{e}^{+2 i \operatorname{Re}\left(k_{z, i}\right) z}\right]\right\}
\end{array}\right],
$$

et finalement

$$
\mathbf{P}(\beta, z)=\frac{1}{2 \omega \epsilon_{0}}\left[\begin{array}{c}
\beta \operatorname{Re}\left(n_{i}^{*} / n_{i}\right)\left\{\left|E_{i \uparrow}\right|^{2} \mathrm{e}^{-2 \operatorname{Im}\left(k_{z, i}\right) z}+\left|E_{i \downarrow}\right|^{2} \mathrm{e}^{+2 \operatorname{Im}\left(k_{z, i}\right) z}\right. \\
\left.-2 \operatorname{Re}\left[E_{i \uparrow} E_{i \downarrow}^{*} \mathrm{e}^{+2 \mathrm{Re}\left(k_{z, i}\right) z}\right]\right\} \\
\operatorname{Re}\left(k_{z, i} n_{i}^{*} / n_{i}\right)\left\{\left|E_{i \uparrow}\right|^{2} \mathrm{e}^{-2 \operatorname{Im}\left(k_{z, i}\right) z}-\left|E_{i \downarrow}\right|^{2} \mathrm{e}^{+2 \operatorname{Im}\left(k_{z, i}\right) z}\right\} \\
+2 \operatorname{Im}\left(k_{z, i} n_{i}^{*} / n_{i}\right) \operatorname{Im}\left[E_{i \uparrow} E_{i \downarrow}^{*} \mathrm{e}^{+2 i \operatorname{Re}\left(k_{z, i}\right) z}\right]
\end{array}\right] .
$$

À partir des équations (A.4, A.8), le calcul du flux $\Phi_{\|}$, moyennant une intégration de $P_{x}$ sur $z$, ne pose pas de problème particulier. 


\section{Annexe B : Atténuation liée à la divergence du faisceau guidé}

Dans notre montage, l'étendue transversale $\ell(400 \mu \mathrm{m})$ de la source dans le plan est égale à la longueur de tranche de guide sur laquelle la lumière issue du guide est collectée, comme l'illustre la figure 56. En outre, comme nous l'avons mentionné en $\S 3.4$., chapitre 4, la collection est effectuée par un objectif de microscope d'ouverture numérique de 0,28 .

En conséquence, lorsque la source est très proche $(L \simeq 0)$, toute l'intensité lumineuse $I_{0}$ émise dans des angles inférieurs à une valeur $\theta_{\mathrm{obj}}\left(\theta_{\mathrm{obj}}=13^{\circ} \mathrm{car}\right.$ $n_{\mathrm{c}}=1,2$ ), est collectée. Lorsque la distance $L$ augmente, une partie de cette lumière n'est plus collectée car elle aboutit hors de la zone analysée, comme l'illustre la figure 56.

Si nous posons $L_{0}=\ell / \tan \theta_{\text {obj, }}$ on montre que l'intensité effectivement collectée s'écrit en fonction de $L$ :

$$
I(L)=\left\{\begin{array}{ll}
I_{0}\left(1-\frac{1}{2} L / L_{0}\right) & 0 \leq L<L_{0} \\
I_{0}\left(\frac{1}{2} L_{0} / L\right) & L \geq L_{0}
\end{array} .\right.
$$

L'atténuation associée est illustrée sur la figure 57. Sur notre montage, $L_{0} \simeq 1,7 \mu \mathrm{m}$, et les mesures sont menées pour des valeurs de $L$ atteignant $1,2 \mu \mathrm{m}$. Les données présentées dans ce manuscrit sont donc systématiquement corrigées en conséquence, afin de ne pas attribuer aux pertes ce qui n'est qu'une conséquence de la divergence du faisceau.

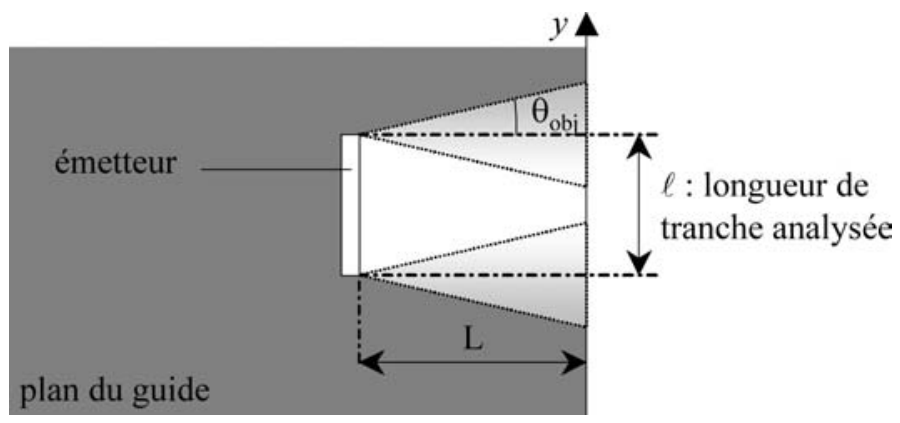

Figure 56. Vue de dessus du faisceau guidé. L'angle $\theta_{\text {obj }}$ est lié à l'ouverture de l'objectif de collection.

[Top view of the guided beam. The angle $\theta_{\mathrm{obj}}$ is related to the aperture of the collecting objective.] 


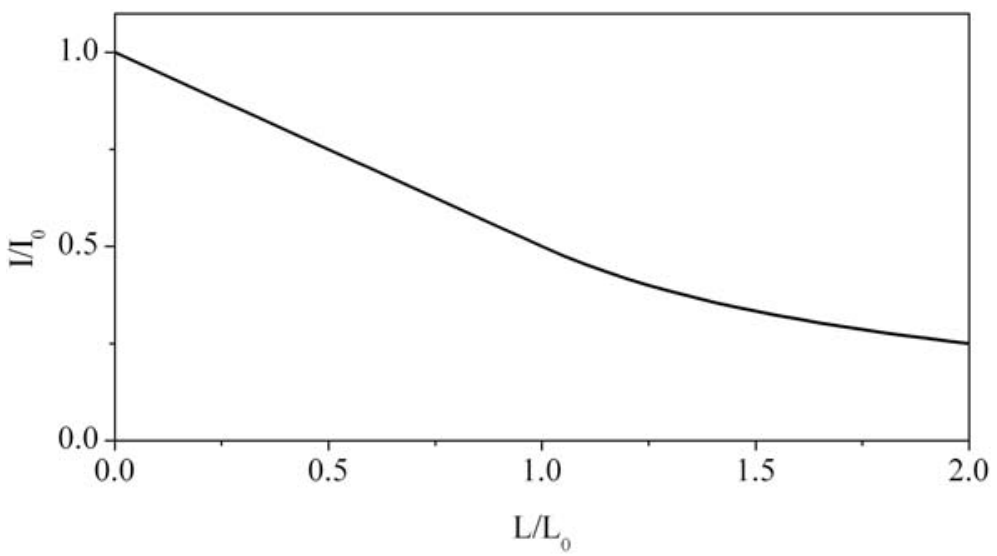

Figure 57. Atténuation de l'intensité mesurée en fonction de la distance $L$ de la source. Nous avons posé $L_{0} \simeq \ell / \tan \theta_{\mathrm{obj}}$.

[Decay of the measured intensity $v$ s. $L$. We set $L_{0} \simeq \ell / \tan \theta_{\text {obj. }}$ ]

À noter que cette correction est inutile lorsque nous injectons un faisceau de lumière blanche : la source est alors très peu étendue, si bien que toute l'intensité émergeant du guide peut être collectée, malgré la divergence. 


\section{Références}

[1] G.E. Moore, Electronics 38, 114-117 (1965).

[2] G.E. Moore, Proc. IEEE 86, 82-85 (1998).

[3] J.D. Joannopoulos, R.D. Meade, J.N. Winn, Photonic Crystals (Princeton : Princeton University Press, 1995).

[4] P. Yeh, Optical Waves in Layered Media (New York: Wiley, 1988).

[5] E. Yablonovitch, Phys. Rev. Lett. 58, 2059-2062 (1987).

[6] E.M. Purcell, Phys. Rev. 69, 681 (1946).

[7] A. Mekis, J.C. Chen, I. Kurland, S. Fan, P.R. Villeneuve, J.D. Joannopoulos, Phys. Rev. Lett. 77, 3787-3790 (1996).

[8] S.Y. Lin, V.M. Hietala, L. Wang, E.D. Jones, Opt. Lett. 21, 1771-1773 (1996).

[9] B. Gralak, S. Enoch, G. Tayeb, J. Opt. Soc. Am. A 17, 1012-1020 (2000).

[10] T.F. Krauss, R.M. De La Rue, S. Brand, Nature 383, 699-702 (1996).

[11] S.Y. Lin, J.G. Fleming, J. Lightwave Technol. 17, 1944-1947 (1999).

[12] A. Blanco, E. Chomski, S. Grabtchak, M. Ibisate, S. John, S.W. Leonard, C. Lopez, F. Meseguer, H. Miguez, J.P. Mondia, G.A. Ozin, O. Toader, H.M. van Driel, Nature 405, 437-440 (2000).

[13] T. Maka, D.N. Chigrin, S.G. Romanov, C.M. Sotomayor Torres, 3-D photonic crystals in the visible regime, in PIER Special Issue on Electromagnetic Applications of Photonic Band Gap Materials and Structures, A. Priou, T. Itoh, Eds., J. Electromagn. Waves Appl. (Cambridge, MA : EMW Publishing, 2002 , in press).

[14] S. Setzu, P. Ferrand, R. Romestain, Mat. Sci. Engineer. B 69-70, 34-42 (2000).

[15] G. Lérondel, R. Romestain, J.C. Vial, M. Thönissen, Appl. Phys. Lett. 71, 196-198 (1997).

[16] J.D. Jackson, Classical Electrodynamics (New York : Wiley, 1975).

[17] D. Marcuse, Theory of Dielectric Optical Waveguides, Quantum Electronics Principles and Applications (San Diego : Academic Press, 1991).

[18] N.W. Ashcroft, N.D. Mermin, Solid State Physics (Philadelphia : Saunders College, 1976). 
[19] D. Kossel, J. Opt. Soc. Am. 56, 1434 (1966).

[20] P. Yeh, A. Yariv, C.S. Hong, J. Opt. Soc. Am. 67, 423-438 (1977).

[21] S.V. Kartalopoulos, Introduction to DWDM technology: data in a rainbow (Washington : SPIE Press, 1999).

[22] D. Cassagne, Ann. Phys. Fr. 23, 1-91 (1998).

[23] M. Plihal, A.A. Maradudin, Phys. Rev. B 44, 8565-5871 (1991).

[24] M.E. Zoorob, M.D.B. Charlton, G.J. Parker, J.J. Baumberg, M.C. Netti, Nature 404, 740-743 (2000).

[25] K.M. Ho, C.T. Chan, C.M. Soukoulis, Phys. Rev. Lett. 65, 3152-3155 (1990).

[26] E. Yablonovitch, T.J. Gmitter, K.M. Leung, Phys. Rev. Lett. 67, 2295-2298 (1991).

[27] Porous Silicon Science and Technology, J.C. Vial, J. Derrien, Eds. (Berlin, Heidelberg : Springer, 1995).

[28] P.M. Fauchet, J. Lumin. 70, 294-309 (1996).

[29] A.G. Cullis, L.T. Canham, P.D.J. Calcott, J. Appl. Phys. 82, 909-965 (1997).

[30] D. Kovalev, H. Heckler, G. Polisski, F. Koch, Phys. Stat. Sol. (b) 215, 871-932 (1999).

[31] Properties of Porous Silicon, L.T. Canham, Ed., Vol. 18 of EMIS datareviews series (London : INSPEC, 1997).

[32] A. Uhlir, Bell Syst. Techn. J. 35, 333 (1956).

[33] D.R. Turner, J. Electrochem. Soc. 105, 402 (1958).

[34] R. Memming, G. Schwandt, Surf. Sci. 4, 109-124 (1966).

[35] M.J.J. Theunissen, J. Electrochem. Soc. 119, 351-360 (1972).

[36] Y. Arita, K. Kato, I. Sudo, IEEE Trans. Electr. Dev. 24, 756-757 (1977).

[37] L.T. Canham, Appl. Phys. Lett. 57, 1046-1048 (1990).

[38] V. Lehmann, U. Gösele, Appl. Phys. Lett. 58, 856-858 (1991).

[39] P. Allongue, V. Costa Kieling, H. Gericher, Electrochim. Acta 40, 1353 (1997).

[40] R.L. Smith, S.D. Collins, J. Appl. Phys. 71, R1-R22 (1992).

[41] M.I.J. Beale, J.D. Benjamin, M.J. Uren, N.G. Chew, A.G. Cullis, J. Cryst. Growth 73, 622-636 (1985).

[42] A. Valance, Phys. Rev. B 55, 9706-9715 (1997).

[43] K. Barla, R. Hérino, G. Bomchil, J.C. Pfister, A. Freund, J. Cryst. Growth. 68, 727-732 (1984).

[44] I. Mihalcescu, J.C. Vial, R. Romestain, Phys. Rev. Lett. 80, 3392-3395 (1998).

[45] A. Halimaoui, C. Oulès, G. Bomchil, A. Bsiesy, F. Gaspard, R. Hérino, M. Ligeon, F. Müller, Appl. Phys. Lett. 59, 304-306 (1991). 
[46] D. Kovalev, H. Heckler, B. Averboukh, M. Ben-Chorin, M. Schwartzkoppf, F. Koch, Phys. Rev. B 57, 3741-3744 (1998).

[47] C. Pickering, M.J. Beale, D.J. Robinson, P.J. Pearson, R. Greef, J. Phys. C: Sol. State Phys. 17, 6535-6552 (1984).

[48] W. Theiss, S. Henkel, M. Arntzen, Thin Solid Films 255, 177-180 (1995).

[49] I. Mihalcescu, G. Lérondel, R. Romestain, Thin Solid Films 297, 245-249 (1997).

[50] D. Kovalev, M. Ben-Chorin, J. Diener, F. Koch, A.L. Efros, M. Rosen, N.A. Gippius, S.G. Tikhodeev, Appl. Phys. Lett. 67, 1585-1587 (1995).

[51] P. Lavallard, R.A. Surris, Solid State Commun. 95, 267-269 (1995).

[52] J. von Behren, P.M. Fauchet, E.H. Chimowitz, C.T. Lira, Optical properties of free-standing ultrahigh porosity silicon films prepared by supercritical drying, in Advances in Microcystalline and Nanocrystalline Semiconductors 1996, R.W. Collins, P.M. Fauchet, I. Shimizu, J.C. Vial, T. Shimada, A.P. Alivisatos, Eds., Vol. 452 of Mater. Res. Soc. Symposium Proceedings, Pittsburgh, pp. 565-570, Material Research Society, 1997.

[53] L.A. Balagurov, D.G. Yarkin, E.A. Petrova, A.F. Orlov, S.N. Karyagin, Appl. Phys. Lett. 69, 2852-2854 (1996).

[54] G. Lérondel, F. Madéore, R. Romestain, F. Müller, Thin Solid Films 366, 216-224 (2000).

[55] G. Vincent, Appl. Phys. Lett. 64, 2367-2369 (1994).

[56] V. Pellegrini, A. Tredicucci, C. Mazzoleni, L. Pavesi, Phys. Rev. B 52, R14328-R14331 (1995).

[57] N. Noguchi, I. Suemune, Appl. Phys. Lett. 62, 1429-1431 (1993).

[58] S. Létant, J.C. Vial, J. Appl. Phys. 80, 7018-7022 (1996).

[59] A.V. Alexeev-Popov, S.A. Gevelyuk, Y.O. Roizin, D.P. Savin, Solid State Commun. 97, 591-593 (1996).

[60] S. Setzu, Réalisation et étude de structures à modulation d'indice optique en silicium poreux, thèse de doctorat, université J. Fourier Grenoble 1, 1999.

[61] U. Grüning, V. Lehmann, S. Ottow, K. Busch, Appl. Phys. Lett. 68, 747-749 (1996).

[62] S. Rowson, A. Chelnokov, J.M. Lourtioz, J. Lightwave Technol. 17, 1989-1995 (1999).

[63] O. Belmont, D. Bellet, Y. Bréchet, J. Appl. Phys. 79, 7586-7591 (1996).

[64] A. Bsiesy, J.C. Vial, F. Gaspard, R. Hérino, M. Ligeon, F. Müller, R. Romestain, A. Wasiela, A. Halimaoui, G. Bomchil, Surf. Sci. 254, 195200 (1991).

[65] D.E. Aspnes, A.A. Studna, Phys. Rev. B 27, 985-1009 (1983).

[66] G. Lérondel, R. Romestain, S. Barret, J. Appl. Phys. 81, 6171-6178 (1997). 
[67] G. Lérondel, R. Romestain, Appl. Phys. Lett. 74, 2740-2742 (1999).

[68] D. Labilloy, H. Benisty, C. Weisbuch, T.F. Krauss, R.M. De La Rue, V. Bardinal, R. Houdré, U. Oesterle, D. Cassagne, C. Jouanin, Phys. Rev. Lett. 79, 4147-4150 (1997).

[69] H. Benisty, R. Stanley, M. Mayer, J. Opt. Soc. Am. A 15, 1192-1201 (1998).

[70] S. Haroche, D. Kleppner, Phys. Today 42, 24-30 (1989).

[71] G. Björk, Y. Yamamoto, Spontaneous emission in dielectric planar microcavities, in Spontaneous emission and laser oscillations in microcavities, $\mathrm{H}$. Yokoyama, K. Ujihara, Eds. (Laser and Optical Science and Technology Series, London : CRC Press, 1995), pp. 189-235.

[72] H. Benisty, H. De Neve, C. Weisbuch, IEEE J. Quant. Electron. 34, 16121631 (1998).

[73] H. Benisty, H. De Neve, C. Weisbuch, IEEE J. Quant. Electron. 34, 16321643 (1998).

[74] J.M. Gérard, B. Sermage, B. Gayral, B. Legrand, E. Costard, V. Thierry-Mieg, Phys. Rev. Lett. 81, 1110-1113 (1998).

[75] R. Matloob, R. Loudon, S.M. Barnett, J. Jeffers, Phys. Rev. A 52, 4823-4838 (1995).

[76] G. Polisski, B. Averboukh, D. Kovalev, F. Koch, Appl. Phys. Lett. 70, 1116-1118 (1997).

[77] D. Kovalev, B. Averboukh, M. Ben-Chorin, F. Koch, A.L. Efros, M. Rosen, Phys. Rev. Lett. 77, 2089-2092 (1996).

[78] S. Setzu, G. Lérondel, R. Romestain, J. Appl. Phys. 84, 3129-3133 (1998).

[79] R. Grousson, V. Voliotis, P. Lavallard, M.L. Roblin, R. Planel, Semicond. Sci. Technol. 8, 1217-1225 (1993).

[80] J.E. Midwinter, Limitations to optical communications, in Confined Photons Systems: Fundamentals and Applications, H. Benisty, J.M. Gérard, R. Houdré, J. Rarity, C. Weisbuch, Eds., Vol. 531 of Lecture Notes in Physics (Berlin, Heidelberg: Springer, 1999), pp. 426-481.

[81] B. Chu, Laser Light Scattering: Basic Principles and Practice, Quantum Electronics - Principles and Applications (San Diego : Academic Press, 1991).

[82] P.K. Tien, Appl. Opt. 10, 2395-2413 (1971).

[83] K.K. Lee, D.R. Lim, H.C. Luan, A. Agarwal, J. Foresi, L.C. Kimerling, Appl. Phys. Lett. 77, 1617-1619 (2000).

[84] A. Yariv, Quantum Electronics (New York: Wiley, 1988).

[85] E. Daub, P. Würfel, Phys. Rev. Lett. 74, 1020-1023 (1995).

[86] G. Vincent, F. Leblanc, I. Sagnes, P.A. Badoz, A. Halimaoui, J. Lumin. 57, 217-221 (1993). 
[87] D. Kovalev, G. Polisski, M. Ben-Chorin, J. Diener, F. Koch, J. Appl. Phys. 80, 5978-5983 (1996).

[88] G. Allan, C. Delerue, M. Lannoo, E. Martin, Phys. Rev. B 52, 11982-11988 (1995).

[89] H. Münder, C. Andrzejak, M.G. Berger, U. Klemradt, H. Lüth, R. Hérino, M. Ligeon, Thin Solid Films 221, 27-33 (1992).

[90] H.F. Arrand, T.M. Benson, P. Sewell, A. Loni, R.J. Bozeat, R. Arens-Fischer, M. Krüger, M. Thönissen, H. Lüth, IEEE J. Select. Top. Quant. Electron. 4, 975-982 (1998).

[91] P. Yeh, A. Yariv, Opt. Commun. 19, 427-430 (1976).

[92] Y. Fink, J.N. Winn, S. Fan, C. Chen, J. Michel, J.D. Joannopoulos, E.L. Thomas, Sci. 282, 1679-1682 (1998).

[93] D.N. Chigrin, A.V. Lavrinenko, D.A. Yarotsky, S.V. Gaponenko, Appl. Phys. A 68, 25-28 (1999).

[94] R.F. Cregan, B.J. Mangan, J.C. Knight, T.A. Birks, P.St.J. Russell, P.J. Roberts, D.C. Allan, Science 285, 1537-1539 (1999).

[95] S.G. Johnson, J.D. Joannopoulos, Opt. Expr. 8, 173-190 (2001).

[96] N. Vorozov, L. Dolgyi, V. Yakovtseva, V. Bondarenko, M. Balucani, G. Lamedica, A. Ferrari, G. Vitrant, J.E. Broquin, T.M. Benson, H.F. Arrand, P. Sewell, Electron. Lett. 36, 722-723 (2000).

[97] A. Yariv, IEEE J. Quant. Electron. 9, 919-933 (1973).

[98] H. Stollh, A. Yariv, Opt. Commun. 8, 5-8 (1973).

[99] S. Olivier, M. Rattier, H. Benisty, C. Weisbuch, C.J.M. Smith, R.M. De La Rue, T.F. Krauss, U. Oesterle, R. Houdré, Phys. Rev. B 63, 113311-1-113311-4 (2001).

[100] J.B. Pendry, A. MacKinnon, Phys. Rev. Lett. 69, 2772-2775 (1992).

[101] P.M. Bell, J.B. Pendry, L. Martín Moreno, A.J. Ward, Comp. Phys. Commun. 85, 306-322 (1995).

[102] M.L. Dakss, L. Kuhn, P.F. Heidrich, B.A. Scott, Appl. Phys. Lett. 16, 523-525 (1970).

[103] H. Kogelnik, T. Sosnowski, Bell Syst. Techn. J. 49, 1602-1608 (1970).

[104] H. Rigneault, F. Lemarchand, A. Sentenac, J. Opt. Soc. Am. A 17, 1048-1058 (2000).

[105] H. Henley, Y. Koshka, J. Lagowski, J. Siejka, J. Appl. Phys. 87, 7848-7852 (2000).

[106] V.P. Parkhutik, L.T. Canham, Phys. Stat. Sol. (a) 182, 591-598 (2000). 


\section{Le silicium nanoporeux : microstructuration diélectrique et application aux structures photoniques avancées}

P. Ferrand

On imagine volontiers le silicium nanoporeux comme une éponge. Pourtant, en raison de la taille nanométrique de ses pores, il constitue aux longueurs d'onde de l'optique un matériau homogène dont l'indice optique peut être ajusté en contrôlant la porosité. Cette propriété, associée à un procédé de fabrication rapide et peu couteux, en fait un candidat prometteur pour la réalisation de microstructures photoniques.

Dans cette étude, nous explorons différents types de microstructures photoniques planaires, en nous concentrant sur la propagation de lumière dans le plan, propagation que nous contrôlons par une structuration périodique submicronique.

Cet ouvrage s'adresse principalement au chercheur confirmé intéressé par les dernières avancées en termes de réalisation de structures photoniques en silicium nanoporeux, mais aussi à l'étudiant de troisième cycle, qui y trouvera sous forme concise et didactique une présentation générale de ce matériau, ainsi que des concepts de base des structures dites à bande interdite photonique. 